Florida International University FIU Digital Commons

FIU Electronic Theses and Dissertations

University Graduate School

$3-22-2012$

\title{
Development and Testing of a Second Generation Hand-held Optical Imager
}

Jean Gonzalez

Florida International University, Jgonz007@fiu.edu

DOI: $10.25148 /$ etd.FI12050203

Follow this and additional works at: https://digitalcommons.fiu.edu/etd

\section{Recommended Citation}

Gonzalez, Jean, "Development and Testing of a Second Generation Hand-held Optical Imager" (2012). FIU Electronic Theses and Dissertations. 596.

https://digitalcommons.fiu.edu/etd/596

This work is brought to you for free and open access by the University Graduate School at FIU Digital Commons. It has been accepted for inclusion in FIU Electronic Theses and Dissertations by an authorized administrator of FIU Digital Commons. For more information, please contact dcc@fiu.edu. 


\title{
FLORIDA INTERNATIONAL UNIVERSITY \\ Miami, Florida
}

\section{DEVELOPMENT AND TESTING OF A SECOND GENERATION HAND-HELD OPTICAL IMAGER}

A thesis submitted in partial fulfillment of the

\author{
Requirements for the degree of \\ MASTER OF SCIENCE \\ in
}

\section{BIOMEDICAL ENGINEERING}

by

Jean Gonzalez 
To: Dean Amir Mirmiran

College of Engineering and Computing

This thesis, written by Jean Gonzalez, and entitled Development and Testing of a Second Generation Hand-held Optical Imager, having been approved in respect to style and intellectual content, is referred to you for judgment.

We have read this thesis and recommend that it be approved.

Wei-Chiang Lin

Anthony Mcgoron

Anuradha Godavarty, Major Professor

Date of Defense: March 22, 2012

The thesis of Jean Gonzalez is approved.

\begin{tabular}{r} 
Dean Amir Mirmiran \\
College of Engineering and Computing \\
\hline Dean Lakshmi N. Reddi \\
University Graduate School
\end{tabular}

Florida International University, 2012 


\section{ABSTRACT OF THE THESIS \\ DEVELOPMENT AND TESTING OF A SECOND GENERATION HAND-HELD OPTICAL IMAGER \\ by \\ Jean Gonzalez}

Florida International University, 2012

Miami, Florida

\section{Professor Anuradha Godavarty, Major Professor}

Hand-held optical imagers are developed towards clinical breast cancer imaging. Herein, a Gen2 hand-held optical imager has been developed with unique features: (i) image curved breast tissues with $\sim 86 \%$ surface contact, and (ii) perform reflectance and transillumination imaging using the novel forked probe heads. Extensive phantom studies were performed using $1 \%$ Liposyn solution (background, $\sim 300 \mathrm{ml}$ and $1000 \mathrm{ml}$ volumes) and $0.45 \mathrm{cc}$ India Ink (absorption) targets, under different target:background contrast ratios and target depths. Twodimensional surface images detected target(s) up to $2.5 \mathrm{~cm}$ deep via reflectance imaging, and up to $5 \mathrm{~cm}$ deep via transillumination imaging. Preliminary studies on gel-based breast phantoms $(\sim 700 \mathrm{ml})$ detected targets via reflectance and transillumination imaging. Preliminary in-vivo reflectance studies on normal and cancerous breast tissues also detected targets, although with artifacts. In future, the portable Gen-2 imager has potential for clinical breast imaging via reflectance and transillumination approach after extensive in-vivo studies. 


\section{TABLE OF CONTENTS}

CHAPTER

PAGE

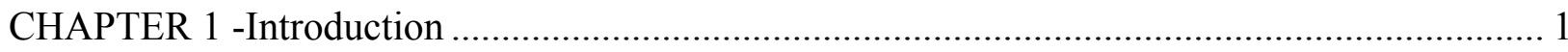

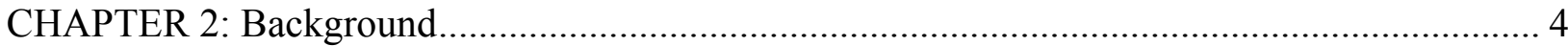

2.1 Basic information on near-infrared optical imaging ................................................... 4

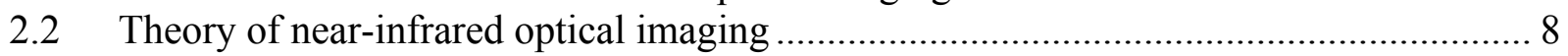

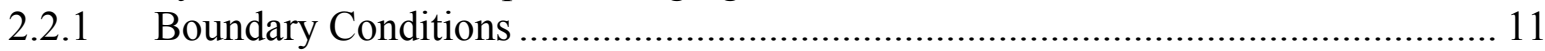

2.2.2 Forward and Inverse Problem ..................................................................... 12

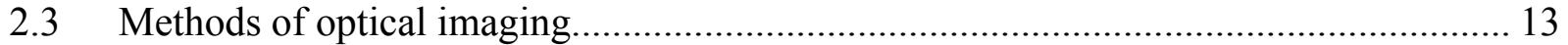

2.4 Measurement Techniques of Optical Imaging ......................................................... 15

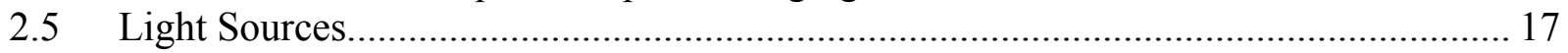

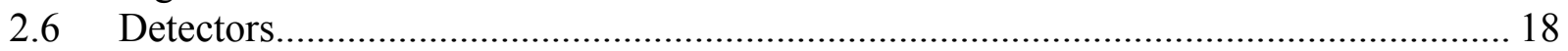

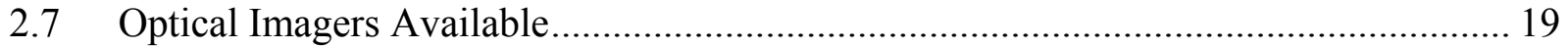

2.8 Optical Imaging Laboratory's First Generation Hand-held Optical Imager.................... 24

2.9 Optical Imaging lab Gen-1 hand-held optical imager Instrumentation.......................... 25

2.10 Principal of Imaging of the Gen-1 hand-held optical imager......................................... 28

2.11 Drawbacks and solutions for the Gen-1 hand-held optical imager ................................ 29

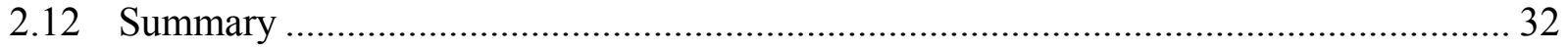

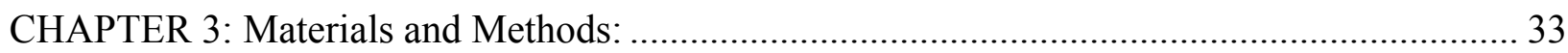

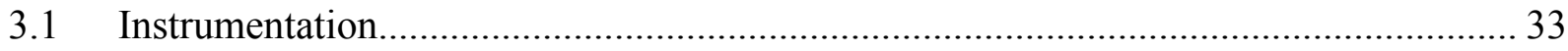

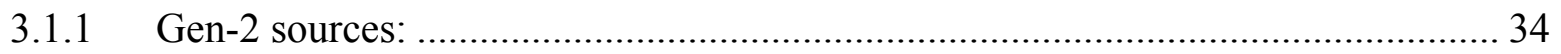

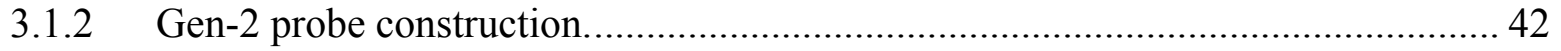

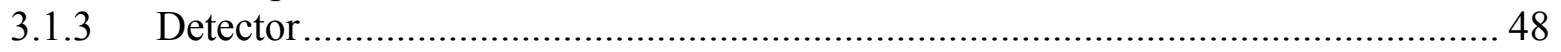

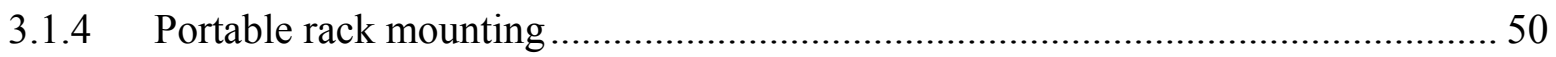

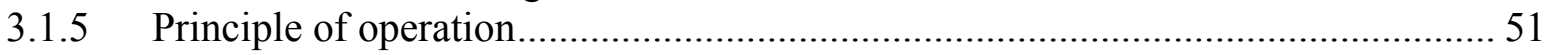

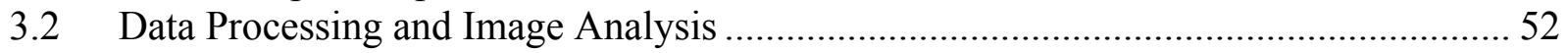

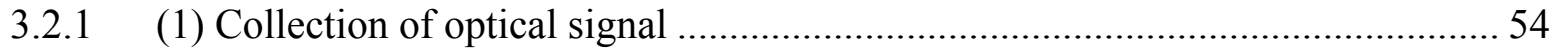

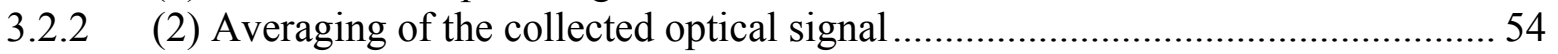

3.2 .3 (3) Extraction and placement of optical intensity measurement.............................5 54

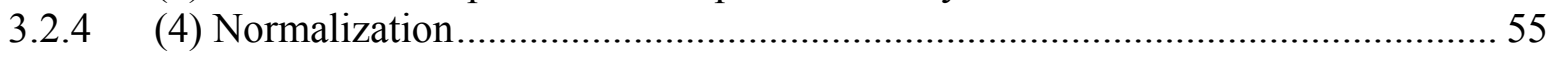

3.2.5 (5) Subtraction Technique..................................................................................... 56

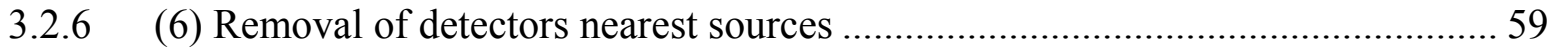

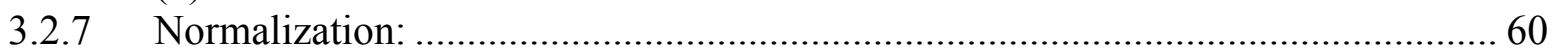

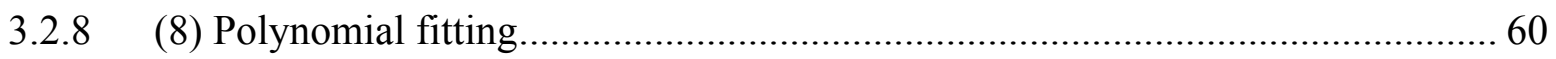

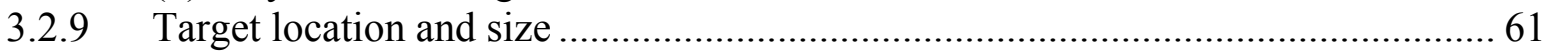

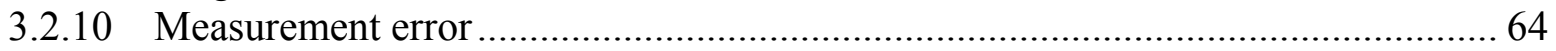

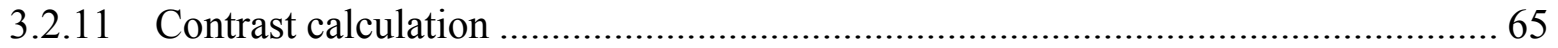

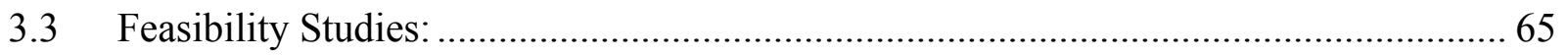

3.3.1 Feasibility of dual probe imaging using a fluorescence target ................................. 65

3.3.2 Feasibility of single probe imaging of an absorption target ................................... 67

3.3.3 Feasibility of dual probe bilateral imaging of an absorptive target (Bilateral

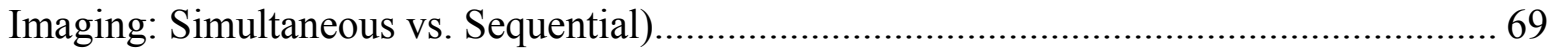




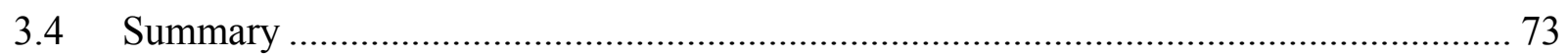

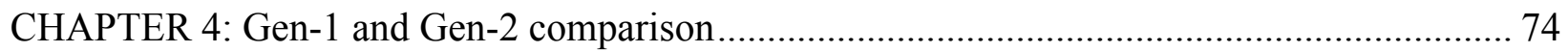

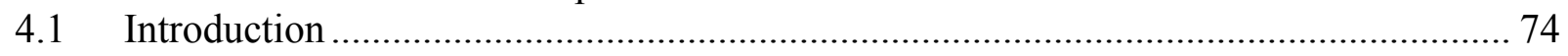

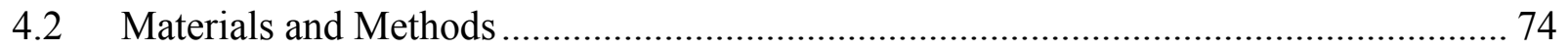

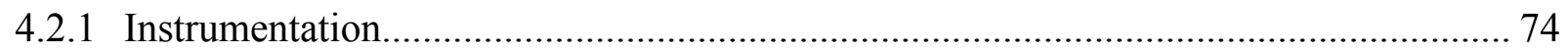

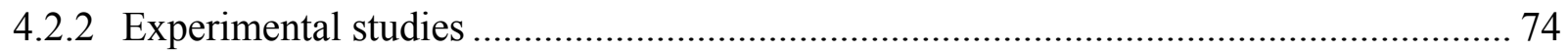

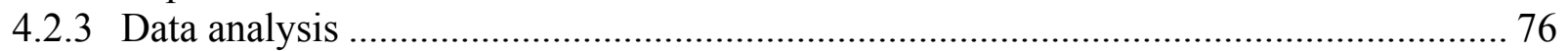

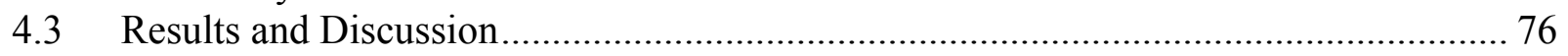

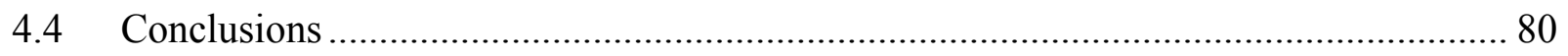

CHAPTER 5: Bilateral and Transillumination imaging on cubical phantoms ............................... 81

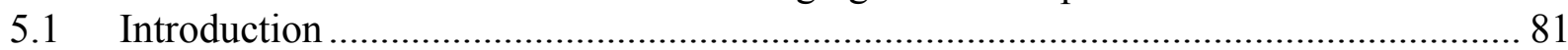

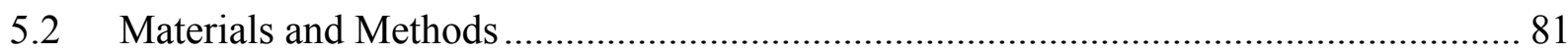

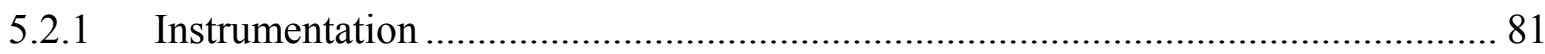

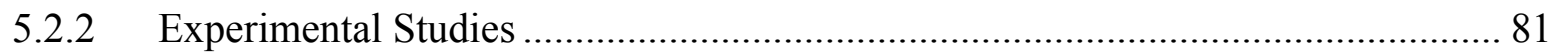

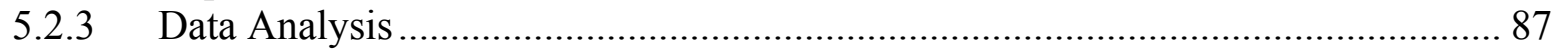

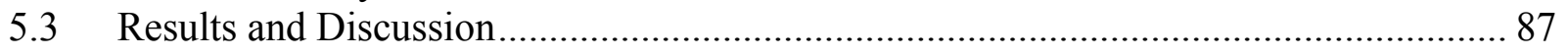

5.3.1 Comparison of reflectance vs. transillumination: Reflectance .............................. 95 96

5.3.2 Comparison of reflectance vs transillumination: Transillumination ....................... 97

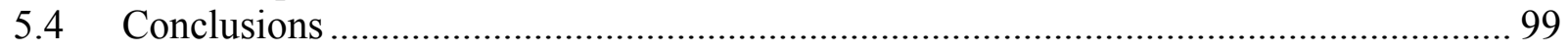

CHAPTER 6: Reflectance and Transillumination imaging of curved tissue phantoms ............. 100

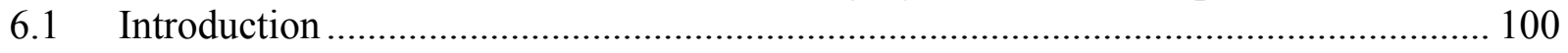

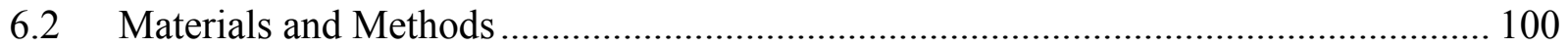

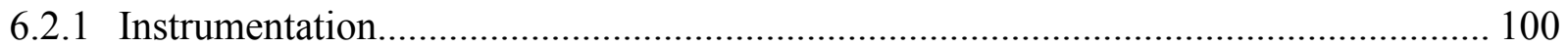

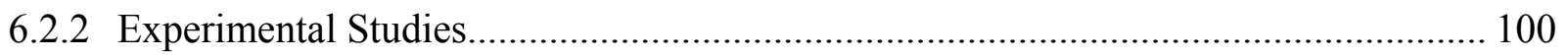

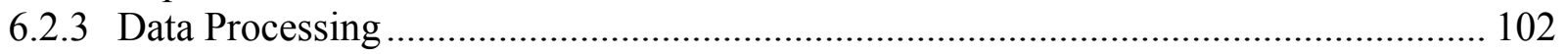

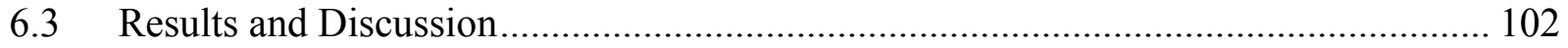

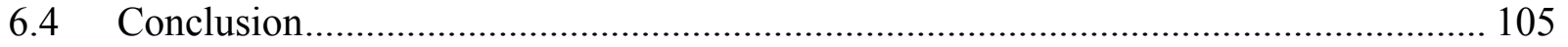

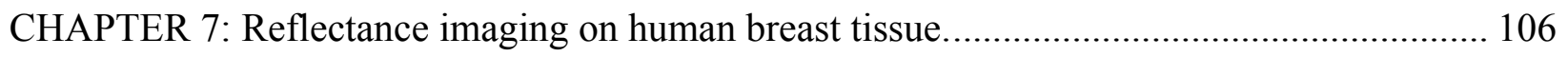

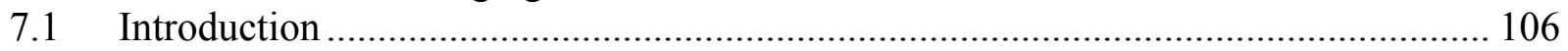

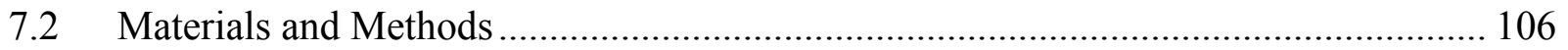

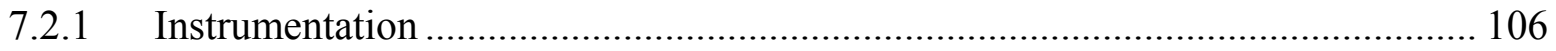

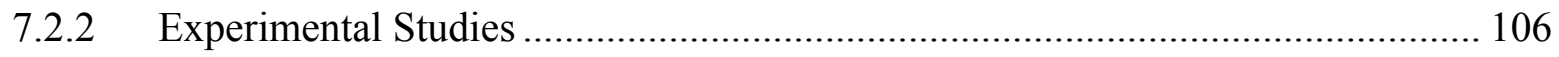

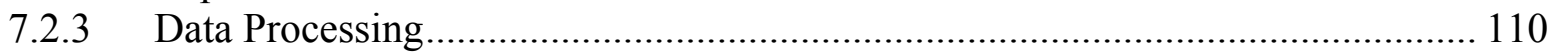

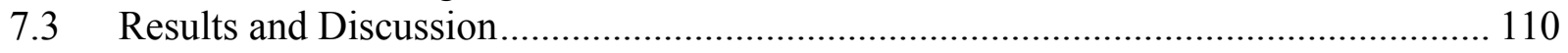

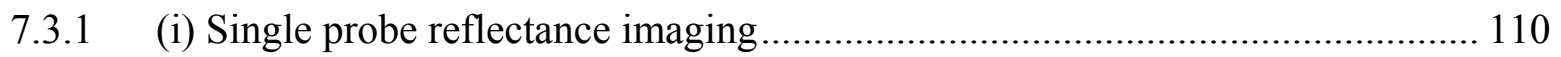

7.3.2 (ii) Effects of pressure on human subject imaging. .............................................. 112

7.3.3 (iii) Simultaneous bilateral imaging on a human subject...................................... 113

7.3.4 (iv) Preliminary in-vivo breast cancer imaging ................................................... 116

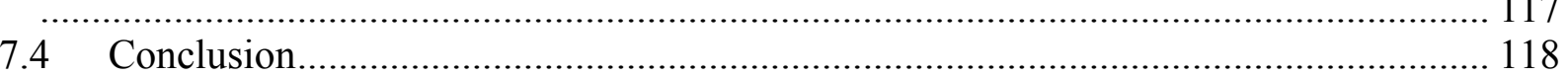




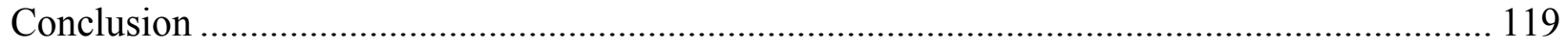

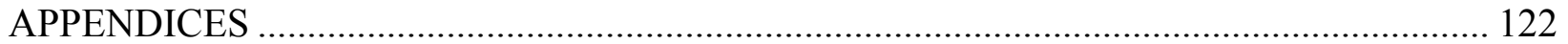

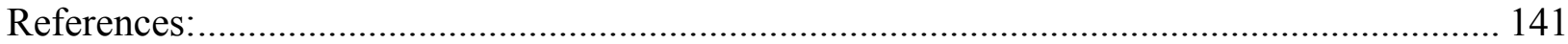




\section{LIST OF FIGURES}

FIGURE

PAGE

Figure 1: Spectrum of absorption from 200-5000 nm. The biological optical Imaging window allows deeper penetration of light from the wavelengths of around 700-900 nm due to minimal absorption by the tissue components, $\mathrm{HbO}, \mathrm{Hb}$ and $\mathrm{H} 2 \mathrm{O}$ in this wavelength range. Reproduced from [33]....

Figure 2: Photons traveling through tissue can reach the same destination using different paths. Ballistic photons travel without any scattering events. Snake photons are minimally scattered. Diffuse photons are heavily scattered. Collection of light at specific locations increases the likelyhood of detectiong one type of photon as opposed to another. Adapted from [32].....

Figure 3: In the forward problem, initial source fluence and optical properties are known the resultant fluence is calculated. In the inverse problem the input (or source) and output (or detected) fluence are known (or measured) and the optical properties are calculated. Adapted from [35].

Figure 4: The 3 methods of applying optical imaging are (A) shadow projection (compressed transillumination), (B) circular tomography (uncompressed transillumination) and (c) subsurface (reflectance) Reproduced from [7].

Figure 5: Reflectance imaging is when is light is launched and collected from the same side.... 14

Figure 6: Transillumination is when light is launched and collected on different surface (example: opposite surface).

Figure 7: In Continuous Wave (CW) the source provides the same output over time. B) Time domain uses a short duration pulse of light. C) Frequency domain (FD) relies on modulating a source output like a sinusoidal wave. Reproduced from [30] ............................................. 16

Figure 8: First Generation hand-held optical imaging system, with the (a) flexible 3-part handheld probe, and (b) the imaging system set-up as a bench-top unit. Reproduced from [30]...... 25

Figure 9: A) Full length view of the hand-held probe of the Gen-1 imager. B) Bundle of optical detection fibers. C) Probe face of the Gen-1 hand-held optical imager D) Source detector layout of the Gen-1 hand-held optical imaging probe. Adapted from [30].

Figure 10: Schematic of the Gen-2 hand-held optical imager. The three main components of the imager are shown (source, detector and hand-held probes)....

Figure 11: The source of the Gen-2 imager is driven by the multichannel controller that is a combination laser driver (LD) and thermoelectric cooler driver (TECD), which allows for the control of up to 8 independent laser mounts. 
Figure 12: (A) laser mount TCLDM9,(B) laser diode 785P100,(C) C230TME-B - f=4.51 mm, $\mathrm{NA}=0.55$, mounted Geltech aspheric Lens, AR: 600-1050 nm,(D) S1TM09 - SM1 to M9 x 0.5 lens cell adapter, (E) SM1SMA - SM1 (1.035"-40) to SMA fiber connector adapter plate,........ 36

Figure 13: Multichannel controller and the laser mounts prior to stability testing with thermal electric driver active. B) Optical power meter (can measure optical power from the laser mounts).

Figure 14: Input current vs. optical power for the six independent channels with their respective laser diodes. 38

Figure 15: The final configuration of the laser mounts, on a rack mountable stage. The mounts are labeled with their channel number (1-6). 39

Figure 16: Newport 8008 Labview driver computer control

Figure 17: Final Automation result (Front Panel) of the computer controlled multichannel controller

Figure 18: The source detector layout of the hand-held optical imagers (a) Gen-1 (source mean and std $1.02 \pm 0.937 \mathrm{~mW}$ ) (b) Gen-2 (source mean and std $2.05 \pm 0.060 \mathrm{~mW}$ )..................... 43

Figure 19: Optical detection fiber bundle of the Gen-2 imaging probe.

Figure 20: (A) Gen-2 hand-held probe face (5 part design). (B) Model of the Gen-2 hand-held probe flat (aerial view), (C) Model of the Gen-2 hand-held probe curved (aerial view), (D) The Gen-2 hand-held probe flat, (E) The Gen-2 hand-held probe curved.

Figure 21: The experimental measurements of curvature for the Gen-1 (a) and 2 (b) hand-held optical probes resulted in values of $\sim 30,86 \%$ using a $13 \mathrm{~cm}$ solid cylindrical phantom respectively.

Figure 22: (A)The PI-Max II is a ICCD camera produced by Princeton instruments towards the detection of low intensity light, (B) the Gen-2 camera within the enclosure for the hand-held optical imager, (C) Optics employed in conjunction with PI-Max II, such as lens tubes and other lens adapters.

Figure 23: The Gen-2 hand-held optical imager is constructed inside of a portable cart (labels indicate the location of hardware).

Figure 24: Flowchart of the steps of data processing. 53

Figure 25: Illuminated optical fiber bundle of the Gen-2 imager as captured via the ICCD (detector). The points selected are calibration points required to match the location of the optical fiber on the fiber bundle with the corresponding end on the probe face. 55 
Figure 26: 2D surface contour plots of a $0.45 \mathrm{cc}$ spherical target placed inside of a liquid liposyn phantom generated through the various steps of the data processing procedure. (A)The unsubtracted (A) and subtracted (B), (C) Subtracted data with the detectors nearest the sources removed and (D) polynomial fitted data of subtracted data.

Figure 27: Subtraction is an optical processing technique to enhance image contrast that centers around the removal of the target to generate a difference between the target (step 1) and background (step 2) images.

Figure 28: Data processing for bilateral imaging. Simultaneous and sequential bilateral imaging apply subtraction processing using the contralateral breast as a background. 59

Figure 29: (A) 2D surface contour plots of the excitation leakage of the Gen-2 hand-held optical imager. (B) Removal of the detectors nearest the points of illumination $\sim 0.5 \mathrm{~cm}$ reduces the excitation leakage from the sources to the detector points and increases homogeneity of the points submitted to the next processing step polynomial fitting. 60

Figure 30: (A) 2D surface contour plot of a $0.45 \mathrm{cc}$ target embedded into a liposyn solution after processing. $(\mathrm{B}, \mathrm{C})$ The column and row of the $2 \mathrm{D}$ surface contour plot based on the centroid or the point of maximum intensity. (D) The data generated from the column and row data in $(\mathrm{cm})$ is the estimated location, Full width at half maximum or estimated size and the $r$ squared when comparing the original row and column data to the 2D-surface contour plot.

Figure 31:Flowchart for the calculation of measurement error 64

Figure 32: Dual probe imaging setup on a single phantom 66

Figure 33: 2D surface contour plot of the detected fluorescence intensity of a cubical tissue phantom containing a $0.45 \mathrm{cc} 1 \mu \mathrm{M}$ ICG target (fluorescence) target at $(8.5,2,2) \mathrm{cm}$ of the liposyn phantom.

Figure 34: Single Probe imaging setup on a single phantom. 68

Figure 35: 2D surface contour plot of detected optical intensity of a cubical phantom containing a India Ink target $(0.08 \%$ by volume $)$ inside a liposyn solution, where the true trarget location is demarcated by the black circle is located at (A) $1.5 \mathrm{~cm}$ deep target, (B) $2.0 \mathrm{~cm}$ deep target....... 68

Figure 36: Bilateral imaging setup on two phantoms 70

Figure 37: Bilateral Imaging of two identical tissue phantoms with embedded India Ink 0.02\% target filled with $1 \%$ liposyn solution. The images are $2 \mathrm{D}$ contour plots of NIR detected signal which result from simultaneous and sequential processing. 
Figure 38: The Gen-1 imager produced 2D surface plots after processing of the optical signal collected during an experimental study involving a $0.45 \mathrm{cc}$ India ink target placed in a cubical $10 \times 10 \times 10 \mathrm{~cm}^{3}$ phantom filled with $1 \%$ liposyn. The target was placed at a) $\left.(2,2.5,1) \mathrm{cm} \mathrm{b}\right)$ $(4,2.5,1) \mathrm{cm}$ and c) $(9,2.5,1) \mathrm{cm}$.

Figure 39: The Gen-2 imager produced the 2D surface plots after processing of the optical signal from an experimental study involving a $0.45 \mathrm{cc}$ India ink target placed in a cubical $10 \times 10 \times 10$ $\mathrm{cm}^{3}$ phantom filled with $1 \%$ liposyn. The target was placed at a) $\left.(1,2.5,1) \mathrm{b}\right)(2.5,2.5,1)$, and c) $(4,2.5,1)$.......

Figure 40: Schematic of experimental tissue phantom study set-up for (A) simultaneous bilateral reflectance imaging, and (B) transillumination imaging 85 Figure 41: 2D surface contour plots (Reflectance imaging) for a $0.45 \mathrm{cc}$ spherical target filled with a $0.8 \%$ by volume India ink. The target was placed in a $10 \times 10 \times 10 \mathrm{~cm}^{3}$ acrylic liposyn phantom with a India ink to make the contrast between target and background 1:0, 1000:1, 100:1 and 10:1.where the black circle represents true target location 88

Figure 42: 2D surface contour plots (Reflectance imaging) for a $0.45 \mathrm{cc}$ spherical target filled with a $0.08 \%$ by volume India ink. The target was placed in a $10 \times 10 \times 10 \mathrm{~cm}^{3}$ acrylic liposyn phantom containing India ink making the T:B contrast ratio 1:0, 1000:1, 100:1 and 10:1.where the black circle represents true target location.

Figure 43: 2D surface contour plots (Reflectance imaging) for a $0.45 \mathrm{cc}$ spherical target filled with a $0.008 \%$ by volume India ink. The target was placed in a $10 \times 10 \times 10 \mathrm{~cm}^{3}$ acrylic liposyn phantom containing India ink making the T:B contrast ratio 1:0, 1000:1, 100:1 and 10:1.where the black circle represents true target location.

Figure 44: 2D surface contour plots (Transillumination imaging) for a $0.45 \mathrm{cc}$ spherical target filled with a $0.8 \%$ by volume India ink. The target was placed in a $10 \times 10 \times 10 \mathrm{~cm}^{3}$ acrylic liposyn phantom containing India ink making the T:B contrast ratio 1:0, 1000:1, 100:1 and 10:1.where the black circle represents true target location.

Figure 45: 2D surface contour plots (Transillumination imaging) for a $0.45 \mathrm{cc}$ spherical target filled with a $0.08 \%$ by volume India ink. The target was placed in a $10 \times 10 \times 10 \mathrm{~cm}^{3}$ acrylic liposyn phantom containing India ink making the T:B contrast ratio 1:0, 1000:1, 100:1 and 10:1.where the black circle represents true target location.

Figure 46: 2D surface contour plots (Transillumination imaging) for a $0.45 \mathrm{cc}$ spherical target filled with a $0.008 \%$ by volume India ink. The target was placed in a $10 \times 10 \times 10 \mathrm{~cm}^{3}$ acrylic liposyn phantom containing India ink making the T:B contrast ratio 1:0, 1000:1, 100:1 and 10:1.where the black circle represents true target location.

Figure 47: Two-dimensional surface contour plots (via 5th degree polynomial fit) of reflectance imaging studies of a $0.45 \mathrm{cc}$ spherical target at 1:0 and 100:1 T:B India Ink contrast, located at various depths. The black hollow circle represents the true $2 \mathrm{D}$ target location. 
Figure 48: Two-dimensional surface contour plots (via 5th degree polynomial fit) of transilluminated reflectance imaging studies of a $0.45 \mathrm{cc}$ spherical target at 1:0 and 100:1 T:B India Ink contrast, located at various depths. The black hollow circle represents the true 2D target location.

Figure 49: Diagram of probe placement during the scanning of a gel phantom with reflectance (A) and transillumination (B) imaging. The target location was approximately $1 \mathrm{~cm}$ off from the curved surface, where the arrows indicate the direction of illumination (long arrows) and light collection (short arrows).

Figure 50: The breast mold (A) employed to produce a solid breast phantom (C) which has a major axis of $16 \mathrm{~cm}$ and a minor axis of $11.5 \mathrm{~cm}$ with a total volume of $\sim 700 \mathrm{ml}$. A $0.45 \mathrm{cc}$ India ink target was place $\sim 1 \mathrm{~cm}$ off the surface represented by the red circle.

Figure 51: Single probe reflectance imaging was performed on a gel phantom with shape and optical properties resembling human breast tissue. A $0.45 \mathrm{cc}$ India ink target $0.08 \%$ by volume - demarcated by the dotted circle on the 2D surface contour plots was inserted $1 \mathrm{~cm}$ from the detection surface. Scanning took place at three locations (top to bottom) on the gel phantoms shown by the dotted squares (probe locations) where the red circle represents the target position inside the phantom

Figure 52: Transillumination imaging was performed on a gel phantom with shape and optical properties resembling human breast tissue. A $0.45 \mathrm{cc}$ India ink target $0.08 \%$ by volume demarcated by the dotted circle on the 2D surface contour plots was inserted $1 \mathrm{~cm}$ from the detection surface. Scanning took place at three locations (right to left) on the gel phantoms shown by the dotted squares (the dotted squares represent the location of the two probe involved in transillumination) where the red circle represents the target position inside the phantom .. 105

Figure 53: A single subject with multi focal cancerous legions in the left breast at approximately 2 o'clock (of the nipple region). The X-ray and ultrasound images display two large distinct legions labeled L1 and L2, while a smaller third legion is labeled L3.

Figure 54: 2D surface contour plots of single probe reflectance imaging on breast tissue of three healthy volunteers. The volunteers were asked to place a $0.45 \mathrm{cc}$ India ink target superficially under the flap of the left (subjects left) breast tissue (infra-mammary fold) at the 6 o'clock position (under the nipple region) to simulate the endogenous contrast of a vascular tumor. A single probe was placed on three locations by the subject as to have the target location relative to the probe on the left, middle and right regions as demarcated by the yellow circles rectangles. The target was removed for the collection of the background image to enhance contrast......... 111

Figure 55: 2D surface contour plots of the effects of pressure on artifact generation. A single healthy human subject with a $0.45 \mathrm{cc}$ absorption target $(.08 \%$ by volume India Ink) placed in the infra-mammary fold of the left breast was asked to apply an incremental pressure for a series of 4 images. The pressure ranged from comfortable (P1), slight (P-2), noticeable (P-3), and uncomfortable (P-4) which were left up to the patient's interpretation. 
Figure 56: 2D surface contour plot of intensity distribution of progressive scans across human breast tissue with a $0.45 \mathrm{cc}$ absorption target embedded under left breast flap at 6'o clock position. Provided is the subtracted image prior to calibration, the calibration factor between the two probes , the adjusted subtracted image and the polynomial fitting of the adjusted subtracted intensity data. 115

Figure 57: The Gen-2 hand-held optical imager was employed (single probe reflectance imaging) to image a cancerous legion. The X-ray and ultrasound images display two large distinct legions labeled L1 and L2, while a smaller third benign legion is labeled L3 (Figure 53) in a 51 year old woman with invasive ductal carcinoma in the left breast. The imaging method involved a sequential top to bottom scan collecting 4 images $\sim(1-2) \mathrm{cm}$ spacing (4 images collected).

Figure A58: Mesh used during the simulation studies conducted for the Gen-2 hand-held optical imager, involving maximum recovery depth and investigation of polynomial fitting. The mesh is $5 \times 5 \times 10 \mathrm{~cm}^{3}$.

Figure B59: Simulation result of a target placed at $(2,2,1) \mathrm{cm}(\mathrm{x}, \mathrm{y}, \mathrm{z})$ from the detection surface employing single probe reflectance imaging.

Figure B60: Comparison of polynomial fitting of 7th and 5th degree. The figure shows several peaks for the 7th degree which negate its improved $\mathrm{R}^{2}$ value.

Figure C61: Simulation of a spherical $(0.95 \mathrm{~cm}$ diameter $)$ absorption contrast target at various depths at a background contrast of 1:0. The depths ranged from $1-3.0 \mathrm{~cm}$ at a $0.5 \mathrm{~cm}$ spacing with the center of the circle at location $2.5,2.5 \mathrm{~cm}-\mathrm{x}, \mathrm{y}$ as demarcated by a black circle.

Figure 62: Block diagram of frequency domain homodyne system. Reproduced from reference [53]. 138

Figure 63: The breast shaped mold (provided by Imaging Diagnostic Systems Inc.) showing the (a) top view, and (b) internal hollow structure 139 


\section{LIST OF TABLES}

TABLE

PAGE

Table 1: Advantages and Disadvantages of various imaging modalities commonly used for cancer prognosis and diagnosis [34-35].....

Table 2: List of sources applicable to NIR imaging ......................................................... 17

Table 3: List of detectors applicable to NIR imaging....................................................... 18

Table 4: Comparison of hand-held optical imagers developed to date by various research groups, the table is condensed to only significant changes within the development of the imagers.

Abridged from reference [7]. Acronynms found in the table for brevity: DOI- Diffuse optical imaging. FDOI- Fluorescence diffuse optical imaging, FDOT- Fluorescence diffuse optical tomography, US- Ultrasound, FD- Frequency Domain, CW- Continuous wave, APDAvalanche photodiode, PMT- Photon multiplier tube, CCD- Charge coupled device [2] ........... 22

Table 5: Comparison between existing Hand-held devices VS Gen-1 Hand-held optical imager

Table 6: The drawbacks of the Gen-1 optical imager and solutions on the instrumentation for the Gen-2 optical imager.....

Table 7: Quantitative details of the detected target location, size and measurement errors for different experimental cases using the Gen-1 and Gen-2 imager.

Table 8: Calculated T:B ratios (factoring in the absorption coefficient of liposyn)

Table 9: Experimental studies using reflectance imaging using a $.45 \mathrm{cc}$ India ink target (concentration of India ink included $0.8,0.08$ and $.008 \%$ by volume) on a $10 \times 10 \times 10 \mathrm{~cm}^{3}$ liposyn phantom (1:0,1000:1, 100:1, and 10:1 target to background contrast ratios). Cases where successful target localization (Within $1 \mathrm{~cm}$ of true target location) occurs are displayed by shaded gray.

Table 10: Experimental studies using transillumination imaging using a $.45 \mathrm{cc}$ India ink target (concentration of India ink included $0.8,0.08$ and $.008 \%$ by volume) on a $10 \times 10 \times 10 \mathrm{~cm}^{3}$ liposyn phantom (1:0, 1000:1, 100:1, and 10:1 target to background contrast ratios). Cases where successful target localization (Within $1 \mathrm{~cm}$ of true target location) occurs are displayed by shaded gray.

Table 11: Experimental cases for simultaneous bilateral reflectance and transillumination imaging studies. In all the studies 3\% Liposyn solution was used in the background phantoms and a $0.45 \mathrm{cc}$ target was with $0.08 \%$ India Ink $+3 \%$ Liposyn solution. In experimental studies with the a 100:1 contrast ratio the background contained $0.0008 \%$ India ink by volume. 
Table 12: Table 2. Quantitative details of the detected target location, size, recovered T:B contrast and measurement errors for different experimental cases using the Gen-2 imager towards reflectance imaging studies.

Table 13: Quantitative details of the detected target location, size, recovered T:B contrast and measurement errors for different experimental cases using the Gen-2 imager towards transillumination imaging studies.

Table 14: The calculated, true target location, target size, contrast, and measurement error for all experimental cases of reflectance performed.

Table 15: The calculated, true target location, target size, contrast, and measurement error for all experimental cases of transillumination performed 


\section{ACRONYMS}

NIR-Near infrared imaging

NIRS-Near infrared spectroscopy

DOI- Diffuse optical imaging.

DOT- Diffuse optical tomography

FDOI- Fluorescence diffuse optical imaging,

FDOT- Fluorescence diffuse optical tomography,

US- Ultrasound,

FD- Frequency Domain,

CW- Continuous wave,

APD-Avalanche photodiode,

PMT- Photon multiplier tube,

CCD- Charge coupled device

$\mathrm{T}: \mathrm{B}-$ Target to background

MRI-Magnetic Resonance imaging

US-Ultrasound

Gen-1 Generation 1 hand-held optical imager

Gen-2 Generation 2 hand-held optical imager 


\section{CHAPTER 1 -Introduction}

Near infrared imaging (NIR) (or spectroscopy) is a promising non invasive, non-ionizing technology that is being developed for brain (connectivity analysis, oxygenation, etc), breast (cancer diagnosis and prognosis, etc) and sports medicine. NIR imaging of breast tissue is based upon the endogenous absorption contrast due to the non-specific process of angiogenesis, allowing for distinction between normal and diseased tissue [1-6] (for theory see section 2.1-2.2).

In recent years, optical imagers were developed in an attempt to translate the nearinfrared (NIR) imaging technology to clinic breast imaging [7-28], with maximum patient comfort and portability. Two main types of optical imagers bed-based and hand-held were developed. Bed-based imagers are typically bulkier and have fixed detector spacing, while handheld imagers are smaller. However many hand-held imagers have not been adaptable to different tissues (limited tissue contact), focused on spectroscopic measurements and limited to two dimensional (2D) tumor detections demonstrating tomographic reconstructions when employed in conjunction with Magnetic Resonance Imaging (MRI) or ultrasound (US).

A novel (Generation-1 or Gen-1) hand-held optical imager was recently developed in our Optical Imaging Laboratory [29-30] (see section 2.8). The unique features of this hand-held imager include: (i) flexibility imaging both large surface areas $\left(5 \times 10 \mathrm{~cm}^{2}\right)$ and different tissue curvatures $\left(0^{\circ}-45^{\circ}\right)$ rapidly, (ii) performance of reflectance imaging from the handheld design, similar to ultrasound; and (iii) ability to perform 3D tomographic imaging (using the tracked positional information of the probe during imaging).

However, the Gen-1 optical imager was limited by: (i) contact on curved tissues (leading to false positives), (ii) A single probe head, although flexible to curve up to $45^{\circ}$ is not capable of collecting transilluminated optical signal. (iii) An aluminum based probe head is not patient 
comfortable (i.e. metal feels cold to skin) and (vi) An uneven source distribution prevented target recovery at all points on the probe.

Herein, a Generation-2 (Gen-2) hand-held optical imager is developed as a portable device with improved features appropriate towards (future) in-vivo breast imaging studies and clinical translation of the technology. In the following sections, the instrumentation development of the imager is described (see section 3.1). The effect of the improved instrumentation in enhancing target detectability is experimentally validated from tissue phantom and preliminary human subject studies (see chapters 4-7).

Objective

The objective of this thesis is the development of a second Generation portable handheld optical imager capable of reflectance and transillumination imaging on tissue phantoms and healthy human subjects. With the successful implementation of the technology at the laboratory level, there is potential for the clinical translation towards diagnostic as well as prognostic breast cancer imaging.

Specific task 1: (Chapter 3) Develop the second Generation hand-held optical imaging imager.

Experimental Study \#1: (Chapter 4). Comparison of the Gen-2 imager with the Gen-1 with regards to target detectibility in phantoms.

Experimental Study \#2: (Chapter 5) Comparison of reflectance and transillumination on phantoms to determine maximum depth at which targets can be recovered and at which contrast ratios 
Experimental Study \#3: (Chapter 6) Imaging of curved tissue phantoms using reflectance and transillumination

Experimental Study \#4: (Chapter 7) In-vivo imaging of human breast tissues.

Thesis Layout:

Chapter 2 contains the background work for this thesis, it includes the theory of optical imaging, comparison of optical with other imaging modalities, an overview of other hand-held optical imagers developed to date and a description of the Gen-1 imager complete with modifications for the Gen-2 imager. Chapter 3 contains the instrumentation of the second Generation hand-held optical imaging system. Chapters 4-7 experimental studies focus on the viability and testing of the Gen-2 imager, initially conducted on liquid phantoms and progressing to gel phantoms an finally human subject studies. 


\section{CHAPTER 2: Background}

The background chapter provides an explanation of the basics of near-infrared optical imaging, its theory, measurement approaches, and the description of various optical imaging devices to date. The hand-held optical devices developed to date by various researchers are also described, followed by the motivation to the development of the second Generation (or Gen-2) hand-held optical imager.

\subsection{Basic information on near-infrared optical imaging}

Optical wavelengths extend from about 300-1300 $\mathrm{nm}$. The energy found in these wavelengths is $<10$ electron volts $(\mathrm{eV})$ meaning the energy found in optical wavelengths is nonionizing. Within this optical range is the near infrared (NIR) region which is found from around 700-900 nm [31]. Wavelengths of light from 700-900 $\mathrm{nm}$ are minimally absorbed and preferentially scattered upon interaction with biological breast tissue (figure 1). These particular absorption and scattering coefficients allow for much deeper light penetration than possible at other optical wavelengths. The reason for low absorption is that the main absorbers in physiological tissue water, oxy- and deoxy-hemoglobin absorb less light than at other wavelengths [32]. 


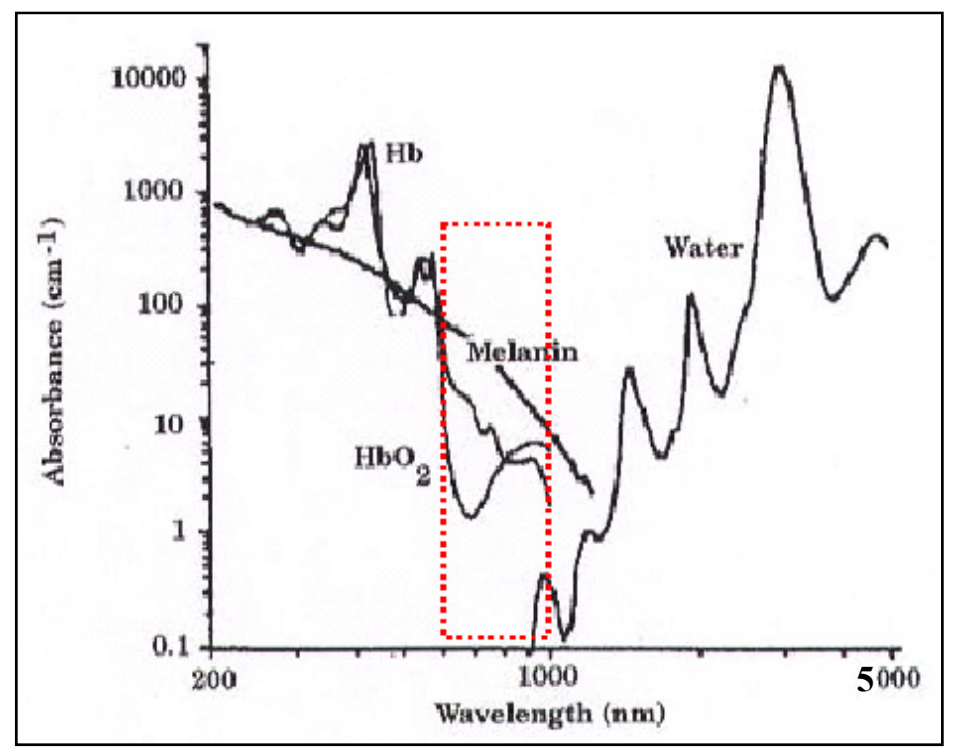

Figure 1: Spectrum of absorption from 200-5000 nm. The biological optical Imaging window allows deeper penetration of light from the wavelengths of around $700-900 \mathrm{~nm}$ due to minimal absorption by the tissue components, $\mathrm{HbO}, \mathrm{Hb}$ and $\mathrm{H} 2 \mathrm{O}$ in this wavelength range. Reproduced from [33]

Deeper tissue penetration allows optical imaging to be implemented as an imaging technology for breast cancer diagnosis and prognosis. Table 1 compares optical with other established imaging modalities [34-35]. The table displays that imaging modalities (with the exception of MRI, optical and ultrasound) submit the patient to radiation exposure to produce the image. Similar to the two extremes of radiation exposure the resolution provided by these imaging modalities ranges from poor (Nuclear, Ultrasound) to excellent (MRI) while providing different types of information. Nuclear, MRI (contrast aided) and optical can provide functional (metabolism and blood flow) information while the rest of the modalities are anatomical in nature (location of hard and soft tissue) including the standard MRI. Imaging time is also variable between the different techniques, with optical, ultrasound and standard x-ray requiring substantially less time than the other modalities. The imaging devices' price ranges are quite wide with optical and ultrasound near the bottom and MRI, and nuclear at the top. Optical 
imaging provides a functional imaging modality with decent resolution and contrast while requiring less imaging time than nuclear and MRI for a less expensive device. The combination of benefits offered by optical imaging in terms of imaging time, resolution, contrast, and cost suggest that optical imaging is a worthwhile technology for cancer diagnosis and prognosis. 


\begin{tabular}{|c|c|c|c|c|c|c|}
\hline & X-Ray & $\mathrm{CT}$ & Ultrasound & MRI & Nuclear & Optical \\
\hline Radiation & Ionizing & Ionizing & Non- Ionizing & Non- Ionizing & Ionizing & Non- Ionizing \\
\hline Resolution & Good & Good & Poor & Excellent & Poor & $\begin{array}{l}\text { Resolution varies } \\
\text { according to depth }\end{array}$ \\
\hline Contrast & Poor & Poor & Poor & Good & Good & Good \\
\hline Portability & Portable & Not Portable & Portable & Not Portable & Not Portable & Portable \\
\hline Expense & Inexpensive & Expensive & Inexpensive & Expensive & Expensive & Inexpensive \\
\hline Information & Structural & Structural & Structural & Structural & Functional & Functional \\
\hline Imaging time & $\begin{array}{l}\text { Fast (less than } 5 \\
\text { min) }\end{array}$ & $\begin{array}{l}\text { Medium (less than } \\
30 \text { min more than } 5 \\
\text { min) }\end{array}$ & $\begin{array}{l}\text { Fast (Less than } 5 \\
\text { min) }\end{array}$ & Slow (over $30 \mathrm{~min}$ ) & Slow (over $30 \mathrm{~min}$ ) & $\begin{array}{lll}\text { Fast (less than } 5 \\
\text { min) } & & \\
\end{array}$ \\
\hline Physics & $\begin{array}{l}\text { High energy } x \text {-rays } \\
\text { travel in a straight } \\
\text { path and are } \\
\text { attenuated by } \\
\text { interaction with } \\
\text { tissue }\end{array}$ & $\begin{array}{l}\text { 3D arrays of } \mathrm{x} \text {-rays } \\
\text { travel in a straight } \\
\text { path and are } \\
\text { attenuated by } \\
\text { interaction with } \\
\text { tissue }\end{array}$ & $\begin{array}{l}\text { Acoustic waves } \\
\text { (mechanical) are } \\
\text { introduced into the } \\
\text { body and are } \\
\text { reflected back } \\
\text { towards a receiver }\end{array}$ & $\begin{array}{l}\text { RF signal is used to } \\
\text { align water } \\
\text { molecules to a } \\
\text { changing magnetic } \\
\text { field and the } \\
\text { resultant RF signal } \\
\text { is collected }\end{array}$ & $\begin{array}{l}\text { High energy } \\
\text { radioactive isotopes } \\
\text { create gamma rays } \\
\text { that travel in a } \\
\text { straight line towards } \\
\text { detectors }\end{array}$ & $\begin{array}{l}\text { Optical light is } \\
\text { introduced into a } \\
\text { tissue and is } \\
\text { reflectance and } \\
\text { absorbed by tissue } \\
\text { interactions }\end{array}$ \\
\hline
\end{tabular}

Table 1: Advantages and Disadvantages of various imaging modalities commonly used for cancer prognosis and diagnosis [34-35]. 
2.2 Theory of near-infrared optical imaging

Biological tissue is a high scattering low absorption medium or high $\mu_{s}$ (probability of photon scattering by a medium per unit path length) low $\mu_{a}$ (probability of absorption by a medium per unit path length). When scattering occurs the photon is directed within a range of possible deflection angles $\mathrm{g}$ (average of cosine of scattering polar angle by single scattering). Figure 2 shows an input light and the possible paths that it can undergo as it travels through a tissue medium. Initially the light undergoes specular reflection, which is the reflection due to the refractive index (n) mismatch between the mediums that the tissue is propagating out of and into ( $\theta$ angle of incoming or reflected light). Snell's equation (1) is used to determine the angle of the reflected light due to the reflectance index mismatch.

$$
\text { [Snells equation } \left.n_{1} \times \theta_{1}=n_{2} \times \theta_{2}\right]
$$

The light transmitted into the tissue undergoes absorption and scattering due to molecules that compose the tissue. Scatterers are molecules that cause the deviation of a photon from the initial path. Examples of scatterers in the body include lipids and proteins. The number of scatterings interactions in tissue is so many that a very small percentage of NIR photons tend to travel straight. Photons are defined by the path taken and could be placed in three categories, ballistic, snake and diffuse [32]. While ballistic photons encounter no scattering interactions, a diffuse photon undergoes various scattering interactions before emerging out of the medium or being completely absorbed. 


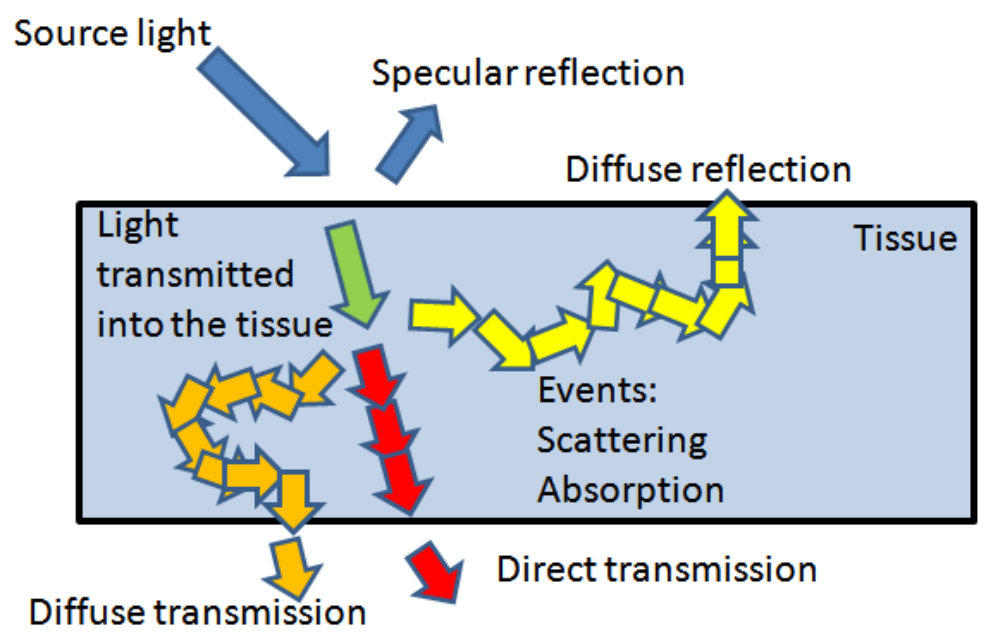

Figure 2: Photons traveling through tissue can reach the same destination using different paths. Ballistic photons travel without any scattering events. Snake photons are minimally scattered. Diffuse photons are heavily scattered. Collection of light at specific locations increases the likelyhood of detectiong one type of photon as opposed to another. Adapted from [32]

By definition a photon is considered diffuse when it has encountered at least 10 scattering events, where the average distance traveled for a single event is the transport mean free path denoted by equation (2).

$$
l_{t}^{\prime}=\frac{1}{\mu_{t}^{\prime}}
$$

where

$\left(l_{t}^{\prime}\right)$-- transport mean free path and,$\left(\mu_{t}^{\prime}\right)$-- is the transport interaction coefficient.

The transport interaction coefficient equation (3) is dependent on reduced scattering coefficient $\left(\mu_{s}^{\prime}\right)$ and absorption coefficient $\left(\mu_{a}\right)$.

$$
\mu_{t}^{\prime}=\mu_{a}+\mu_{s}^{\prime}
$$

The reduced scattering coefficient equation (4) is the probability of equivalent isotropic photon scattering by a medium per unit pathlength. $(g$ )-- anisotropy factor

$$
\mu_{s}^{\prime}=\mu_{s} \times(1-g)
$$


The values of $\mu_{s}^{\prime}$ and $\mu_{a}$ are found prior to an experiment (via a homodyne or heterodyne device) to ensure the values are similar to human breast tissue. The variable $\mu_{s}^{\prime}$ and $\mu_{a}$ represent the optical properties of a medium and can be used to generate the $\mu_{\text {eff }}$ equation (5) which is the exponential decay rate of fluence far from the source.

$$
\mu_{e f f}=\sqrt{3 \mu_{a}\left(\mu_{s}^{\prime}+\mu_{a}\right)}
$$

With the i) $\mu_{s}^{\prime}, \mu_{a}$ (optical properties of the given medium), the assumption of ii) an isotropic (and homogenous) source, iii) high scattering and low absorption medium the propagation of light can be modeled via the photon diffusion equation (Equation 6). Other methods to model the propagation of light included a microscopic approach as is provided using Maxwell's equations which although more fundamental and exact are computationally more expensive. The method of modeling chosen is using the radioactive transport equation, which with several assumptions that ease the computational requirements in biological tissue Ggnerate equation (6) the photon diffusion equation. The assumptions required for Diffusion equations are (i) isotropic source (light is uniform intensity and angles); (ii) $\left(\mu_{\mathrm{s}}^{\prime}>>\mu_{\mathrm{a}}\right.$ probability of scattering is much greater than probability of absorption) where the scattering length is much smaller than the mean free absorption length in the medium; and (iii) the light is diffuse, i.e. at least 10 scattering lengths between the point of illumination to detection

$$
S\left(\mathrm{r}^{\rightarrow}, \mathrm{t}\right)=\frac{\partial \Phi\left(\mathrm{r}^{\rightarrow}, \mathrm{t}\right)}{c \partial t}+\mu_{a} \Phi\left(\mathrm{r}^{\rightarrow}, \mathrm{t}\right)-\nabla \cdot\left[\mathrm{D} \nabla^{2} \Phi\left(\mathrm{r}^{\rightarrow}, \mathrm{t}\right)\right]
$$

where $(\mathrm{t})$-- time,$\left(\mathrm{r}^{\rightarrow}\right)$--direction vector), $\Phi\left(\mathrm{r}^{\rightarrow}, \mathrm{t}\right)$--fluence rate or intensity, $S\left(\mathrm{r}^{\rightarrow}, \mathrm{t}\right)-$-- isotropic source term.

$$
\mathrm{D}=\frac{1}{3}\left(\mu_{s}^{\prime}+\mu_{a}\right)
$$

In equation (7) $\mathrm{D}$ it is the coefficient linking the flow of photons to the gradient of fluence in Fick's law. 


\subsubsection{Boundary Conditions}

Boundary conditions were suggested to represent the tissue boundaries they include (i) reflective index matched boundary, (ii) reflective index mismatched boundary and (iii) zero boundary condition $[32,35]$.

(i) Reflective index matched boundary condition

In the case of a reflectance index matching boundary as with water and soft tissue, no light propagation into the scattering medium from the ambient medium occurs. Hence the fluence rate is set to zero at an extrapolated boundary located at distance $z_{b}$.

$$
\Phi\left(\mathrm{r}^{\rightarrow}, \mathrm{t}\right)-2 \mathrm{D} \frac{\partial \Phi\left(\mathrm{r}^{\rightarrow}, \mathrm{t}\right)}{\partial z}=0
$$

where $\Phi\left(\mathrm{r}^{\rightarrow}, \mathrm{t}\right)=0$ at $z_{b}=2 \mathrm{D}$

(ii) Refractive index mismatched boundary

In the case of a reflectance index mismatch as with tissue and air, the boundary condition is modified to follow Fresnel's reflections. Equation (9) adds the term $C_{R}$ to equation (8) where equations 9-15 display the progression of the terms required to find the term $\left(\mathrm{C}_{\mathrm{R}}\right.$ - index mismatch which is a function of the effective reflectance term $R_{\text {eff }}$ ).

$$
\begin{gathered}
\Phi\left(\mathrm{r}^{\rightarrow}, \mathrm{t}\right)-2 \mathrm{DC}_{\mathrm{R}} \frac{\partial \Phi\left(\mathrm{r}^{\rightarrow}, \mathrm{t}\right)}{\partial z}=0 \\
\mathrm{C}_{\mathrm{R}}=\frac{1+\mathrm{R}_{\mathrm{eff}}}{1-\mathrm{R}_{\mathrm{eff}}}
\end{gathered}
$$

The effective reflectance term (Reff) depends on the $\mathrm{R}_{\theta}$ and $\mathrm{R}_{\mathrm{j}}$ which are determined by the refractive index $\mathrm{n}_{\text {rel }}$.

$$
\begin{gathered}
\mathrm{R}_{\text {eff }}=\frac{\mathrm{R}_{\Phi}+\mathrm{R}_{\mathrm{J}}}{2-\mathrm{R}_{\Phi}+\mathrm{R}_{\mathrm{J}}} \\
\mathrm{R}_{\Phi}=\int_{0}^{\frac{\pi}{2}} 2 \sin \theta \mathrm{R}_{\mathrm{F}}(\cos \theta) \mathrm{d} \theta \\
\mathrm{R}_{\mathrm{J}}=\int_{0}^{\frac{\pi}{2}} 3 \sin \theta(\cos \theta)^{2} \mathrm{R}_{\mathrm{F}}(\cos \theta) \mathrm{d} \theta
\end{gathered}
$$




$$
\begin{gathered}
R_{F}(\cos \theta)=\frac{1}{2}\left(\frac{n_{r e l} \cos \theta^{\prime}-\cos \theta}{n_{r e l} \cos \theta^{\prime}+\cos \theta}\right)^{2}+\left(\frac{n_{r e l} \cos \theta^{\prime}-\cos \theta \prime}{n_{r e l} \cos \theta^{\prime}+\cos \theta}\right)^{2} \\
\theta=\cos ^{-1}\left(n_{r e l} \sin \theta\right) \\
\theta^{\prime}=\sin ^{-1}\left(n_{r e l} \sin \theta\right)
\end{gathered}
$$

$$
\text { where } \Phi\left(\mathrm{r}^{\rightarrow}, \mathrm{t}\right)=0 \text { when } z_{b}=-2 \mathrm{C}_{\mathrm{R}} \mathrm{D}
$$

(iii) Zero boundary condition

In this boundary condition the fluence $\Phi$ is set to zero on and after the boundary (equation 17), which is simpler (in comparison to the other boundary conditions) though it is not an accurate representation of a real system.

$$
\Phi\left(\mathrm{r}^{\rightarrow}, \mathrm{t}\right)=0 \text { at } \mathrm{z}=0
$$

\subsubsection{Forward and Inverse Problem}

In a forward problem, the optical properties for the entire tissue medium are assumed to be known and the fluence is evaluated analytically and numerically for the whole of the tissue medium (figure 3). The result is an output fluence upon the surface boundary where measurements were acquired. 


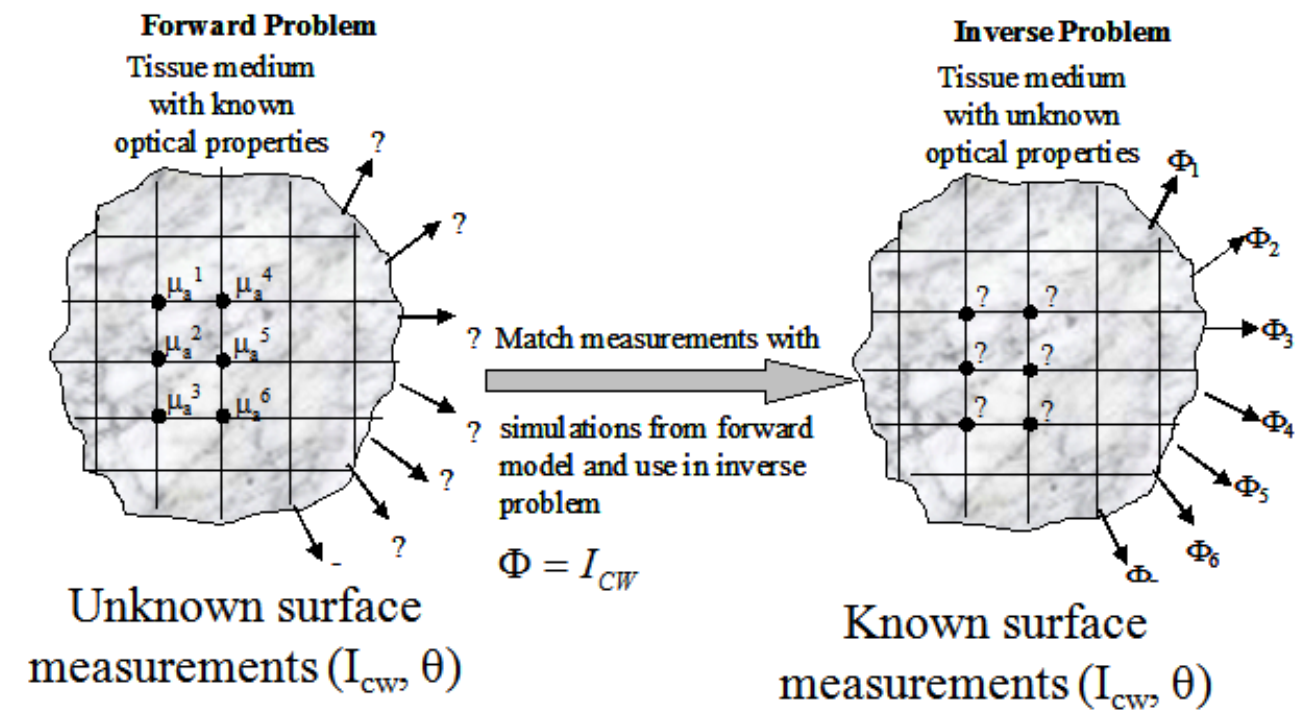

Figure 3: In the forward problem, initial source fluence and optical properties are known the resultant fluence is calculated. In the inverse problem the input (or source) and output (or detected) fluence are known (or measured) and the optical properties are calculated. Adapted from [35].

The inverse problem employs the fluence measured on the surface of the tissue geometry and estimates the optical property map of the entire tissue volume using appropriate light propagation models in tissue and inversion algorithms [32]. The result is a 3D tomographic map of the tissue optical properties that help differentiate between normal and abnormal tissue types in the tissue volume that is imaged.

\subsection{Methods of optical imaging}

Optical imaging requires a light source to be launched onto the tissue surface. As the light propagates, it undergoes interactions explained in section 2.2 until it is collected at the same or opposite tissue surface using appropriate detectors. The three most commonly used imaging methods are (a) projection-shadow, (b) circular tomography and (c) sub-surface imaging as seen on figure 4 [7]. Projection-shadow and circular tomography will be called transillumination and 
differentiated by the tissue geometry (compressed vs. uncompressed) and sub-surface imaging will be referred to as reflectance imaging.

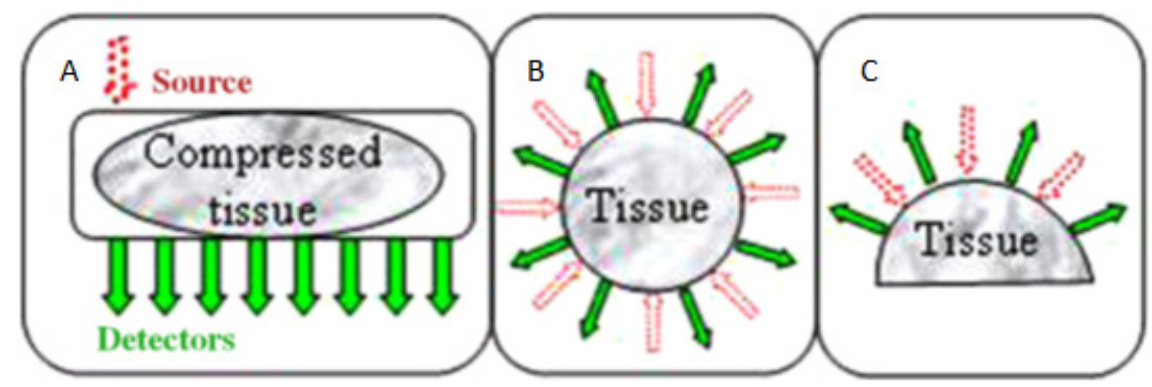

Figure 4: The 3 methods of applying optical imaging are (A) shadow projection (compressed transillumination), (B) circular tomography (uncompressed transillumination) and (c) sub-surface (reflectance) Reproduced from [7].

Reflectance imaging (Figure 4) captures photons that have been scattered in the direction that the light was initially launched. Reflectance imaging allows for a single probe design that can both illuminate the tissue and collect the returning signal.

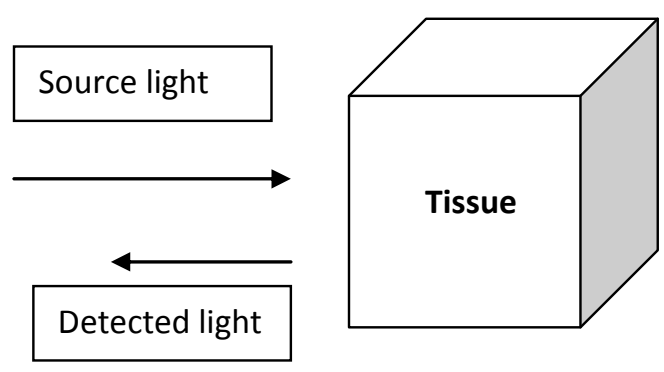

Figure 5: Reflectance imaging is when is light is launched and collected from the same side.

Transillumination imaging is when light is launched and propagates through thickness of the tissue and collected on another side from where it was launched (Figure 6). 


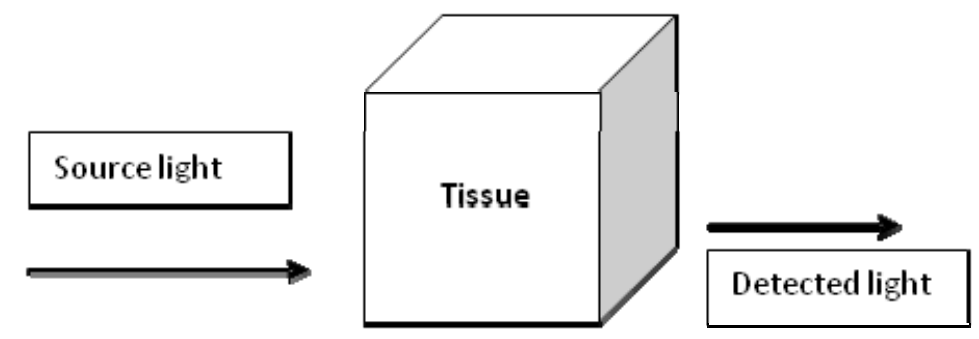

Figure 6: Transillumination is when light is launched and collected on different surface (example: opposite surface).

\subsection{Measurement Techniques of Optical Imaging}

There are three possible techniques in which optical imaging measurements are acquired. (a) Continuous Wave (CW): involves collection of steady state light. The source wavelength and intensity are constant (same $\mathrm{mW}$ output) throughout imaging and the resultant signal is captured using appropriate detectors. $\mathrm{CW}$ is the simplest and hence least expensive and fastest of the three techniques discussed. (b) Frequency Domain (FD): The source and detector are modulated at a given frequency by other instrumentation. The modulated light source then propagates through the tissue and as it travels attenuation will occur towards its amplitude, and its phase will be shifted the further it travels with respect to the reference signal. The modulated attenuated signal is collected by a modulated detector and the amplitude and relative phase shift are measured. The addition of the phase provides additional information resulting in greater image contrast at the expense of increasing imaging time when compared with CW. (c) Time Domain (TD): The source is pulsed and launched onto the tissue. As the pulsed signal propagates, it is attenuated and acquired at the detector with a time lag (that is proportional to the pathlength taken by the photons). The detector measures the attenuated intensity (abu) as well as the time lag with respect to the input pulse. Time domain provides the greatest image enhancement where ballistic photons could be separated out from diffuse at the expense of a much larger imaging time when compared with CW. For this master's thesis a continuous wave system is proposed 
and constructed. A CW system requires less instrumentation than a frequency domain system and does not require the expensive components associated with time domain imaging, thus reducing the expense. CW systems also require less time to collect images while frequency domain requires a moderate increase in time, while time domain requires a large increase $[30,32,35]$.

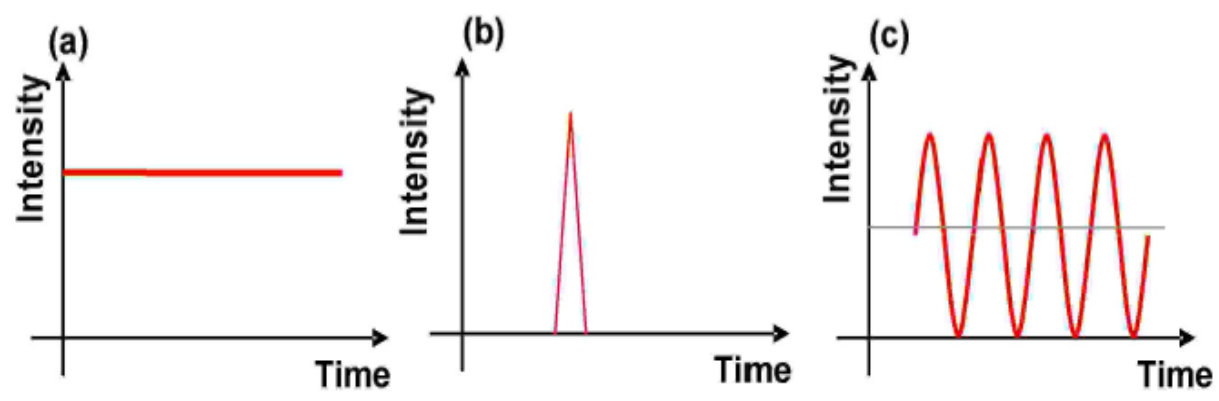

Figure 7: In Continuous Wave (CW) the source provides the same output over time. B) Time domain uses a short duration pulse of light. C) Frequency domain (FD) relies on modulating a source output like a sinusoidal wave. Reproduced from [30] 


\subsection{Light Sources}

NIR imaging can be performed with multiple or single wavelengths, provided by different choices in light sources. Below is a table listing the possible sources that could be employed in NIR imaging (www.Thorlabs.com),(www.edmundoptics.com).

\begin{tabular}{|l|l|}
\hline Sources & Description \\
\hline Lamps. i.e. broad spectrum sources & $\begin{array}{l}\text { Broadband light sources emit a large spectrum of light } \\
\text { that can then be reduced via appropriate filters to the } \\
\text { wavelength of choice. The wide spectrum makes this } \\
\text { the source of choice for spectrum analysis. }\end{array}$ \\
\hline Pulse Lasers & $\begin{array}{l}\text { Pulse lasers can be found at specific wavelengths and } \\
\text { produce a signal that pulses with a set period. }\end{array}$ \\
\hline Tunable lasers & $\begin{array}{l}\text { Tunable lasers provide an accurate, steady, } \\
\text { monochromatic signal. }\end{array}$ \\
\hline LED (light emitting diodes) & $\begin{array}{l}\text { LEDs are an inexpensive source that could be purchased } \\
\text { either monochromatic or broad band. Stability could be }\end{array}$ \\
\hline variable as with any diode device especially if from \\
different silicon wafers.
\end{tabular}

Table 2: List of sources applicable to NIR imaging

The lasers could be paired with various optical component choices depending on the requirements of the user. 


\subsection{Detectors}

Detectors for NIR imaging much like sources are dependent on the imaging technique employed. Table 3 below displays common types of detectors used in NIR imaging.

\begin{tabular}{|c|c|}
\hline Detectors & Description \\
\hline Photomultiplier Tubes (PMT) & $\begin{array}{l}\text { Photomultiplier tubes or (PMT) a vacuum tube } \\
\text { derived device is capable of capturing a light } \\
\text { signal and amplifying it after it has been } \\
\text { converted to an electrical signal. PMT are } \\
\text { applied towards single point detection such as a } \\
\text { single optical fiber. }\end{array}$ \\
\hline Avalanche Photodiodes (APD) & $\begin{array}{l}\text { Avalanche photodiodes use the photoelectric } \\
\text { effect to convert a light signal into an amplified } \\
\text { electrical signal. APD are applied towards } \\
\text { single point detection such as a single optical } \\
\text { fiber. }\end{array}$ \\
\hline Charge Coupled Devices (CCD) & $\begin{array}{l}\text { Collect incoming light converting it to a charge } \\
\text { in a well. The devices pixel values correspond } \\
\text { to the wells. CCDs are applied towards area } \\
\text { detection and can collect light signal from } \\
\text { multiple points. Various types of CCD are } \\
\text { available such as (ICCD-intensified), } \\
\text { (EMCCD-electron multiplying), etc }\end{array}$ \\
\hline
\end{tabular}

Table 3: List of detectors applicable to NIR imaging 


\subsection{Optical Imagers Available}

Optical imagers initially start as bench-top or research systems then during clinical translation, transition into what can be broadly classified into two categories: bed-based and hand-held. Bed-based imagers can use both transillumination and reflectance as the imaging method, while hand-held imagers employ reflectance based imaging method [7].

Bed-based imagers are a category of imagers which require a subject to lie down and place the tissue to be imaged in special imaging bins or enclosures. The advantages of these imagers are listed below: 1) These imagers may use more powerful lasers $(50 \mathrm{~mW})$ than hand-held imagers to increase tissue illumination to facilitate transmission through the length of the tissue [2]; 2) Some of the bed-based designs simplify acquiring and processing the collected data by using techniques such as tissue compression to reduce the thickness of the tissue similar to $\mathrm{x}$-ray mammography [6]; and 3) The built-in fixed source and detector locations help in 3D tomographic imaging, where 3D tumor localization and characterization is feasible. [2,35-37]. The drawbacks of the bed-based systems include: 1) The systems are typically less portable than hand-held imagers due to additional components such as the bed and breast enclosure which add to the overall size of the imager.; 2) The imaging method employed may cause exclusion of some patients populations. These excluded patients may include those with larger breasts than the imaging bins or enclosures employed in the bed-based imagers.

Hand-held imagers provide similar optical measurements as bed-based imagers, but have their own advantages and disadvantages. The advantages of the hand-held imagers are listed below: 1) These imagers can be employed to image a wide range of patient populations without any restrictions to breast volumes and/or sizes; and 2) These imagers are typically smaller in size 
than bed-based imagers and in some cases could be easier to transport and be moved through clinical sites without restrictions.

The hand-held imagers also have limitations, which include: 1) The inability to precisely track the location of the hand-held probe during studies limits the applicability of these imagers towards 3D tomographic imaging as well as accurate tumor tracking from multiple patient visits.

2) The current imaging method for hand-held imagers rely on technicians to acquire images at desired locations in the standardized manner. This would add error to scans by different technicians if the imaging location or method changes across patients. The reason for the difference is probe placement, leading to variations in the true location of the images on the tissue; which in turn can affect the accuracy of tomographic imaging [2,30].

Several bed-based and hand-held imagers have been developed, towards imaging of biological breast tissue. Table 4 lists the current hand-held optical imagers developed, the imaging method which the device employs and the research group. The table includes significant improvements or evolution of each group's imager. From the table it is seen that the imagers developed by several groups use CW-based diffuse optical (DOI) or frequency domain (FD) imaging techniques for quick acquisition of the optical signal. The employment of CW-based DOI or FD imaging techniques require avalanche photodiodes (APD) or photon multiplier tubes (PMT) for collection of optical signal from a point source as is used by the majority of imagers. Only 2 groups applied charge coupled devices (CCD) to facilitate acquisition of multiple point optical signals from various optical fibers. The additional optical fibers decrease the number of scans and imaging time required for optical signal acquisition from a tissue area. Diode lasers are typically implemented in these imagers to reduce cost, size while maintaining production of monochromatic light. One of the imagers displayed includes a second imaging modality using 
ultrasound, which is used to gain anatomical and positional information for the imager yielding enhanced results from only optical. However from the imagers below only FIU's optical imaging laboratory's (OIL) Gen-1 imager has displayed the ability to perform both fast 2D imaging and 3D tomographic imaging. FIU's optical imaging laboratory's (OIL) Gen-1 imager is the topic of the next section. 


\begin{tabular}{|c|c|c|c|c|c|c|}
\hline Device \# & Reference & Modality & $\begin{array}{l}\text { Meas. } \\
\text { Technique }\end{array}$ & Source Type & Detector Type & Clinical Application(s) \\
\hline \multirow[t]{3}{*}{1} & $\begin{array}{l}\text { Tromberg, } \\
1997\end{array}$ & DOI & $\begin{array}{l}\text { FD } \\
(300 \mathrm{kHz}- \\
1 \mathrm{GHz})\end{array}$ & $\begin{array}{l}\text { diode lasers } \\
(10-30 \mathrm{~mW})\end{array}$ & APD & $\begin{array}{l}\text { Compare optical properties of } \\
\text { normal and benign lesion- } \\
\text { containing breast tissue }\end{array}$ \\
\hline & Shah, 2004 & DOI & $\begin{array}{l}\text { FD } \\
(50-700 \\
\mathrm{MHz})\end{array}$ & $\begin{array}{l}\text { diode lasers } \\
(5-25 \mathrm{~mW})\end{array}$ & APD & $\begin{array}{l}\text { Measure optical \& } \\
\text { physiological properties of } \\
\text { healthy breast tissue }\end{array}$ \\
\hline & $\begin{array}{l}\text { Bevilacqua, } \\
2000\end{array}$ & DOI & $\begin{array}{l}\text { CW \& FD } \\
(100-700 \\
\mathrm{MHz})\end{array}$ & $\begin{array}{l}\text { FD - diode lasers } \\
(<20 \mathrm{~mW}) \\
\mathrm{CW}-150 \mathrm{~W} \text { haloGen } \\
\text { lamp }\end{array}$ & $\begin{array}{l}\text { FD - APD } \\
\text { CW - fiber- } \\
\text { coupled } \\
\text { spectrograph w/ } \\
\text { linear CCD } \\
\text { detector }\end{array}$ & $\begin{array}{l}\text { Measure the absorption } \\
\text { spectra of normal human } \\
\text { breast tissue }\end{array}$ \\
\hline \multirow{3}{*}{2} & $\begin{array}{l}\text { Cerussi, } \\
2007\end{array}$ & DOI & $\begin{array}{l}\text { CW \& FD } \\
(50-1000 \\
\mathrm{MHz})\end{array}$ & $\begin{array}{l}\text { FD - diode lasers }(20 \\
\text { mW) } \\
\text { CW - white-light source }\end{array}$ & $\begin{array}{l}\text { FD - APD } \\
\text { CW - fiber- } \\
\text { coupled } \\
\text { spectrograph w/ } \\
\text { linear CCD } \\
\text { detector }\end{array}$ & $\begin{array}{l}\text { Predict postsurgical } \\
\text { pathological response to } \\
\text { neoadjuvant chemotherapy in } \\
11 \text { breast cancer subjects }\end{array}$ \\
\hline & No, 2005 & DOI & $\begin{array}{l}\text { FD } \\
(10 \mathrm{MHz}- \\
1 \mathrm{GHz})\end{array}$ & $\begin{array}{l}8 \text { laser diodes } \\
(50 \mathrm{~mW})\end{array}$ & APD & (Phantom study) \\
\hline & No, 2007 & DOI & $\begin{array}{l}\text { FD } \\
(50-300 \\
\mathrm{MHz})\end{array}$ & $\begin{array}{l}4 \text { laser diodes } \\
(20 \mathrm{~mW})\end{array}$ & APD & $\begin{array}{l}\text { Detect the presence of breast } \\
\text { cancer in a human subject }\end{array}$ \\
\hline 3 & $\begin{array}{l}\text { Chance, } \\
2005\end{array}$ & DOI & $\mathrm{CW}$ & $\begin{array}{l}\text { light emitting diodes } \\
(10-15 \mathrm{~mA})\end{array}$ & $\begin{array}{l}\text { silicon diode } \\
\text { detector }\end{array}$ & $\begin{array}{l}\text { Determine sensitivity and } \\
\text { specificity of detecting breast } \\
\text { cancer in } 116 \text { human subjects }\end{array}$ \\
\hline 4 & $\begin{array}{l}\text { Chance, } \\
2006\end{array}$ & DOI & $\begin{array}{l}\text { FD } \\
(3 \mathrm{kHz})\end{array}$ & $\begin{array}{l}2 \text { light emitting diodes } \\
(20 \mathrm{~mA})\end{array}$ & $\begin{array}{l}\text { silicon diode } \\
\text { detector }\end{array}$ & $\begin{array}{l}\text { Detection and 2D localization } \\
\text { of brest cancer lesion in a } \\
\text { human subject }\end{array}$ \\
\hline 5 & Liu, 2004 & FDOI & $\begin{array}{l}\text { FD } \\
(3 \mathrm{kHz})\end{array}$ & 4 light emitting diodes & PMT & (Phantom study) \\
\hline \multirow[t]{2}{*}{6} & Cheng, 2003 & DOI & $\mathrm{CW}$ & $\begin{array}{l}\text { laser diodes } \\
(0.15 \mathrm{~W} / \mathrm{cm} 2)\end{array}$ & PMT & $\begin{array}{l}\text { Determine sensitivity and } \\
\text { specificity of detecting breast } \\
\text { cancer in } 50 \text { human subjects }\end{array}$ \\
\hline & $\mathrm{Xu}, 2007$ & DOI & $\mathrm{CW}$ & $\begin{array}{l}\text { laser diodes } \\
(0.15 \mathrm{~W} / \mathrm{cm} 2)\end{array}$ & PMT & $\begin{array}{l}\text { Detect tissue optical, } \\
\text { mechanical, and physiologic } \\
\text { changes under dynamic } \\
\text { loading }\end{array}$ \\
\hline 7 & $\begin{array}{l}\text { Durduran, } \\
2005\end{array}$ & DOI & $\mathrm{CW}$ & long coherence laser & APD & $\begin{array}{l}\text { Measure blood flow contrast } \\
\text { between tumor-containing and } \\
\text { normal breast tissues }\end{array}$ \\
\hline \multirow[t]{4}{*}{8} & Zhu, 1999 & $\begin{array}{l}\text { DOT } \\
\& U S\end{array}$ & $\begin{array}{l}\text { FD } \\
(200 \mathrm{MHz})\end{array}$ & laser diodes & APD & $\begin{array}{l}\text { Phantom study towards 3D } \\
\text { tomography of breast cancer }\end{array}$ \\
\hline & Chen, 2001 & $\begin{array}{l}\text { DOT \& } \\
\text { US }\end{array}$ & $\begin{array}{l}\text { FD } \\
(140 \mathrm{MHz})\end{array}$ & laser diodes & PMT & $\begin{array}{l}\text { Phantom study towards 3D } \\
\text { tomography of breast cancer }\end{array}$ \\
\hline & Zhu, 2003 & $\begin{array}{l}\text { DOT \& } \\
\text { US }\end{array}$ & $\begin{array}{l}\text { FD } \\
(140 \mathrm{MHz})\end{array}$ & laser diodes & PMT & $\begin{array}{l}\text { Image benign and malignant } \\
\text { breast lesions in human } \\
\text { subjects }\end{array}$ \\
\hline & Zhu, 2010 & $\begin{array}{l}\text { DOT \& } \\
\text { US }\end{array}$ & FD & laser diodes & PMT & $\begin{array}{l}\text { Differentiate benign and } \\
\text { malignant lesions at early- } \\
\text { stage in breast tissue }\end{array}$ \\
\hline \multirow[t]{2}{*}{9} & $\mathrm{Ge}, 2008$ & FDOT & $\begin{array}{l}\mathrm{FD}(100 \\
\mathrm{MHz})\end{array}$ & laser diode $(<5 \mathrm{~mW})$ & $\mathrm{CCD}$ & $\begin{array}{l}\text { Phantom towards 3D } \\
\text { tomography of breast cancer }\end{array}$ \\
\hline & $\begin{array}{l}\text { Erickson, } \\
2010\end{array}$ & FDOI & $\mathrm{CW}$ & laser diode $(<5 \mathrm{~mW})$ & $\mathrm{CCD}$ & $\begin{array}{l}\text { Fast 2D imaging of } \\
\text { fluorescent targets in breast } \\
\text { tissue of healthy subjects }\end{array}$ \\
\hline 10 & $\begin{array}{l}\text { Solomon, } \\
2010\end{array}$ & FDOT & $\mathrm{CW}$ & $\begin{array}{l}\text { laser diodes }(15-30 \\
\mathrm{mW})\end{array}$ & $\mathrm{CCD}$ & $\begin{array}{l}\text { Phantom \& small animal } \\
\text { study towards sentinel lymph } \\
\text { node mapping }\end{array}$ \\
\hline
\end{tabular}

Table 4: Comparison of hand-held optical imagers developed to date by various research groups, the table is condensed to only significant changes within the development of the imagers. Abridged from reference [7]. 
Acronynms found in the table for brevity: DOI- Diffuse optical imaging. FDOI- Fluorescence diffuse optical imaging, FDOT- Fluorescence diffuse optical tomography, US- Ultrasound, FD- Frequency Domain, CW-

Continuous wave, APD-Avalanche photodiode, PMT- Photon multiplier tube, CCD- Charge coupled device [2] 


\subsection{Optical Imaging Laboratory's First Generation Hand-held Optical Imager}

Translating NIR imaging systems from the bench-top to bedside (either bed-based or portable) requires improvements in patient comfort, portability, ease of use and function. Currently the hand-held imaging systems (hand-held) available have only flat probe heads (Probe head: section of the optical imaging system that contacts the tissue) causing inherent limitations as given in Table 5 [2,38-39].

\begin{tabular}{|l|l|}
\hline $\begin{array}{l}\text { Existing Hand-held optical imagers } \\
\text { (by other research groups) }\end{array}$ & Our Hand-held optical imager \\
tissue curvatures & curvatures \\
\hline $\begin{array}{l}\text { Obtains only reflectance measurements which } \\
\text { limits depth information }\end{array}$ & Obtains reflectance and transillumination \\
measurements from the flexible probe head design, \\
for surface imaging of small areas & towards greater depth information \\
\hline Provide 2D images with no positional & Provides 2D/3D coregistered images in real time \\
coregistration onto tissue & using a motion tracker \\
\hline $\begin{array}{l}\text { Demonstrated 2D/3D tomography only when } \\
\text { employed along with ultrasound }\end{array}$ & Demonstrated 3D tomographic imaging on tissue \\
\hline $\begin{array}{l}\text { Provides only spectroscopic information } \\
\text { phantoms (to date) without a } 2^{\text {nd }} \text { imaging modality }\end{array}$ \\
\hline
\end{tabular}

Table 5: Comparison between existing Hand-held devices VS Gen-1 Hand-held optical imager 
A novel first Generation hand-held optical imaging system was recently developed in our Optical Imaging Laboratory. Figure 8 (red numbers display the major components that will be discussed further in the text) and on Table 3 the advantages over other hand-held optical imaging systems that use flat probes are given.

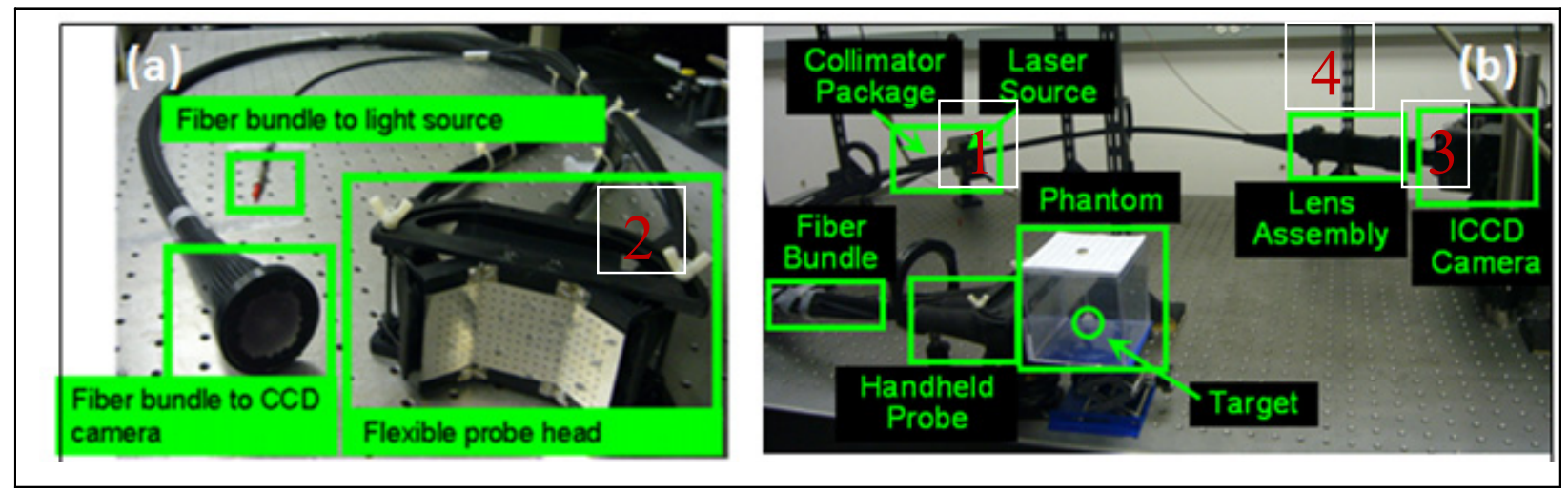

Figure 8: First Generation hand-held optical imaging system, with the (a) flexible 3-part hand-held probe, and (b) the imaging system set-up as a bench-top unit. Reproduced from [30].

2.9 Optical Imaging lab Gen-1 hand-held optical imager Instrumentation

\subsubsection{Gen-1 hand-held optical imager Source: 1}

The first Generation hand-held optical imaging system uses a single laser mount with a laser diode that emits light at $785 \mathrm{~nm}$. The laser mount is driven and cooled by a Laser current controller and temperature controller. A collimator-diffuser package is coupled to the laser diode (and mount) in order to provide homogenous (uniform) source light to six optical fibers that are connected to the diffuser package via a single SMA connector. The source optical fibers are appropriately placed at 6 discrete locations on the probe face. The laser mount also used an RF input that could be modulated via an oscillator, when operating the imaging system in the frequency domain. [30] 


\subsubsection{Gen-1 hand-held optical imager Detector: 2}

The detector employed on the first Generation hand-held optical imaging system is an intensified charge coupled device (ICCD) camera. The first Generation hand-held optical imaging system had the intensifier added (modified in-house after purchase) providing the ability to take low light images. The first Generation hand-held optical imaging system could be operated to acquire either $\mathrm{CW}$ or FD measurements, as described in the principal of imaging (section 2.3). The NIR signals collected from the tissue surface are imaged by the ICCD camera via the fiber bundle (containing 165 collection optical fibers). These intensity images are further post-processed to obtain the actual image of the collected NIR signal at the tissue surface. [30] 

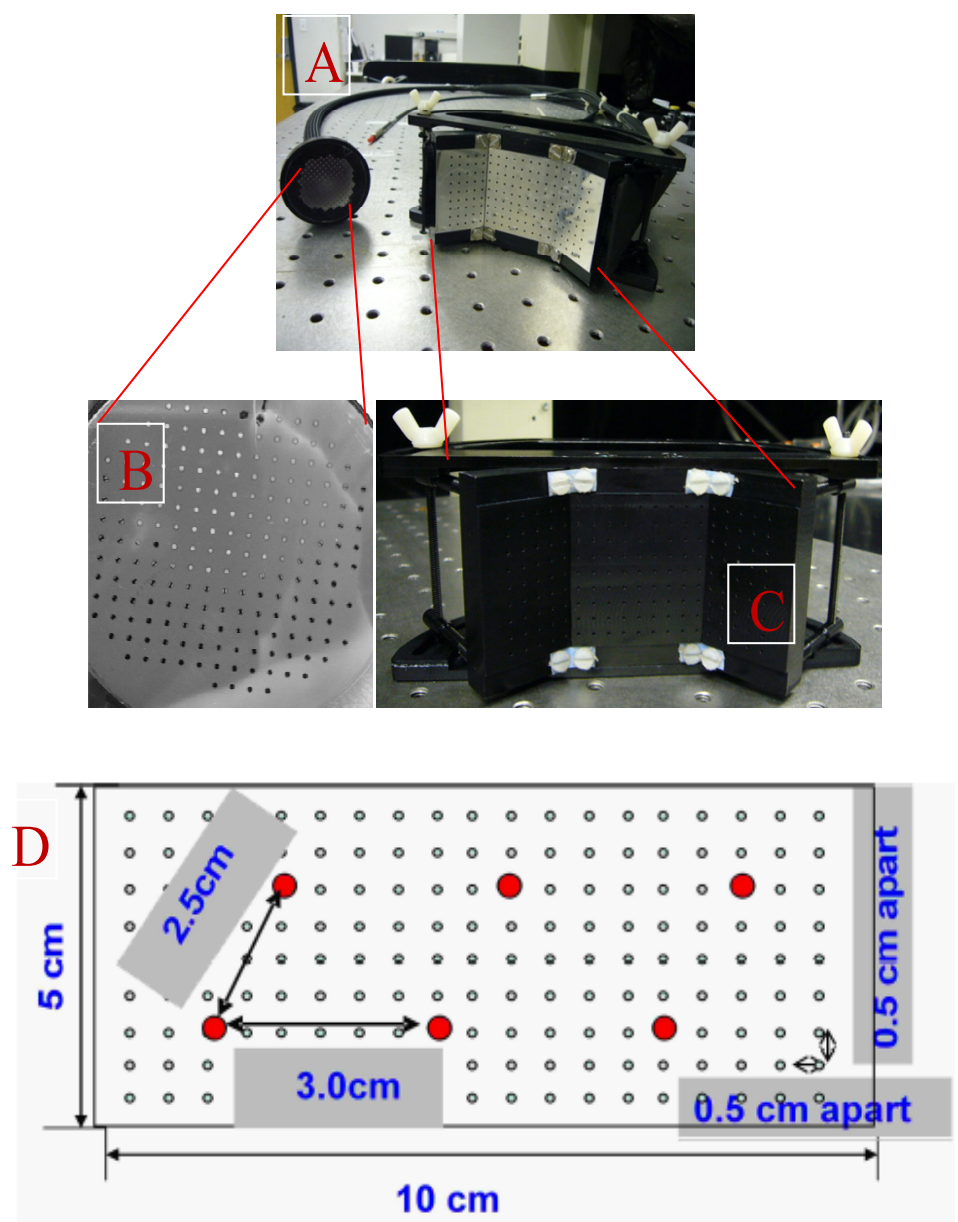

Figure 9: A) Full length view of the hand-held probe of the Gen-1 imager. B) Bundle of optical detection fibers. C) Probe face of the Gen-1 hand-held optical imager D) Source detector layout of the Gen-1 hand-held optical imaging probe. Adapted from [30].

\subsubsection{Gen-1 hand-held optical imager Probe: 3}

The hand-held optical imaging probe makes contact with the tissue surface to acquire NIR intensity signals. The optical probe consists of optical fibers bundled together where one end (Figure 9B) is placed on a circular plate (camera end) and the other is the imaging surface (Figure 9C). The hand-held probe is composed of 165 optical collection fibers and 6 source fibers mounted onto the probe. The hand-held probes imaging surface is capable of flexing each 
of the joints of its three plate design from 0-45 degrees. The spacing between the optical fibers is $0.5 \mathrm{~cm}$. All the details of probe face dimensions are visible in figure $9(\mathrm{~d})$. The material chosen for the probe face was aluminum due to its ease of machining and durability[29,30].

\subsubsection{Gen-1 hand-held optical imager Lens Assembly: 4}

An appropriate lens assembly system is placed between the ICCD detector and the fiber bundle (consisting of the 165 collection fibers). Apart from housing a focusing lens required for a focused image of the fiber bundle, the lens assembly also houses appropriate filters required to acquire NIR signals at same or higher wavelengths (e.g. excitation or fluorescence signals) [30].

\subsection{Principal of Imaging of the Gen-1 hand-held optical imager}

NIR light at $785 \mathrm{~nm}$ is launched from the laser diode, which is split to 6 source fibers after it propagates through the collimator-diffuser package. The six source fibers simultaneously illuminate the tissue surface in contact. The launched source light attenuates and scatters as it propagates through the tissue. The extent of attenuation and scattering depend on the optical properties (absorption and scattering) of the tissue medium. Some of the light is scattered back towards the hand-held probe, which is collected by the 165 collection fibers on the probe face. The NIR signals from the fiber bundle (containing 165 collection fibers) is imaged using the ICCD camera. The steady-state intensity signals are further processed to obtain the true intensity distribution of the tissue surface being imaged. The system could be operated in either $\mathrm{CW}$ or FD mode. The principal of images changes between the two modes. The main difference being that, with $\mathrm{CW}$ mode the lasers and the images are collected at a steady state with no modulation involved. With FD mode, the lasers and the camera are modulated at a specific frequency (100 $\mathrm{MHz}$ in our studies) and the phase between them is changed. Detailed description of the instrumentation for CW and FD mode are provided elsewhere [30].. 
2.11 Drawbacks and solutions for the Gen-1 hand-held optical imager

The first Generation imager was originally designed to test how effective a hand-held optical probe could be used towards target detection. The design proved to be very viable but it has drawbacks as in table $6[38,39]$.

The solutions to the drawbacks of the Gen-1 imager are displayed on table. The overall goal to address specific task 1 (development of the improved hand-held optical imager) was to improve various aspects of imaging using a hand-held imager (improve target detectibility, patient comfort, and increase target depth recovery), thus creating an imager that was more suited towards the clinical environment. The improvements and modifications consisted of (i) improve flexibility and tissue surface contact, (ii) implement a forked design applicable towards bilateral and transillumination imaging, (iii) improve patient comfort, (iv) improve image quality and reduce artifact creation, (v) enhance portability and ease travel within a clinical environment, and (vi) allow for the implementation of a optical tracking system for probe positioning. 


\begin{tabular}{|c|c|}
\hline Gen-1 Drawbacks & Gen-2 Solutions \\
\hline $\begin{array}{l}\text { 1) The hand-held imaging probe is composed up of } 3 \text { segmented flat plates, } \\
\text { in an attempt to allow curve tissue imaging. However having three plates } \\
\text { caused the loss of potential contact leading to a noisier signal which could } \\
\text { lead to false positives in human cancer patients due to specularly reflected } \\
\text { signal at the edges of these } 3 \text { segmented plates. }\end{array}$ & $\begin{array}{l}\text { 1) The hand-held imaging probe was reduced in size and made up of more } \\
\text { than three plates leading to improved flexibility. The potential benefits } \\
\text { include such as conforming to tissue surface areas with minimal } \\
\text { compression and potentially reducing the specularly reflected signal due to } \\
\text { loss of contact with the tissue surface. }\end{array}$ \\
\hline $\begin{array}{l}\text { 2) The hand-held imaging probe is composed of a single probe head, which } \\
\text { was not effective in collecting transillumination images. A single probe } \\
\text { design also causes stress on the optical detection fibers when flexed, thus } \\
\text { preventing it from flexing to contour to tissue geometries. The design was } \\
\text { chosen to image large areas at once but it was determined to be difficult to } \\
\text { place and hold on tissue surfaces for the length of the imaging studies. }\end{array}$ & $\begin{array}{l}\text { 2) The hand-held imaging probe was split from a single face to a forked } \\
\text { probe design which would reduce the size of each probe while keeping the } \\
\text { total imaging area the same. Leading to high quality images due to better } \\
\text { position, and ease of use. The forked design also requires less optical } \\
\text { fibers per probe causing less stress on the optical fibers during positioning. }\end{array}$ \\
\hline $\begin{array}{l}\text { 3) The hand-held imaging probe is composed of an aluminum probe face, } \\
\text { which is machine able and easy to work with in terms of fiber attachment. } \\
\text { However, the thermal transfer properties of the aluminum are not patient } \\
\text { friendly; aluminum feels cold to the touch and could cause discomfort } \\
\text { especially when placed on more sensitive tissues. The technician holding } \\
\text { the device would suffer from fatigue during an experiment, due to the } \\
\text { weight of the Hand-held probe }(\sim 2 \mathrm{lbs}) \text {, thus negatively impacting the } \\
\text { imaging results. }\end{array}$ & $\begin{array}{l}\text { 3) The hand-held imaging probe was manufactured from materials that } \\
\text { prevent quick thermal transfer, and thereby increasing patient comfort with } \\
\text { the imaging process. Also a change of material would also reduce the } \\
\text { weight of the probe possibly reducing technician fatigue associated with } \\
\text { having to place and hold the optical probe during imaging. }\end{array}$ \\
\hline
\end{tabular}


4) The hand-held imaging probe is constructed with a source light derived from a single laser mount that was split into six source optical fibers. However, the collimator-diffuser package used for the source system creates a non uniform intensity distribution, thus impacting the target detectability in cases when the target is small, deep, or of low target:background contrast ratio.

5) The hand-held imager is constructed on an optical bench making an immobile setup, which prevents the imaging process from taking place in a clinic environment.
4) The hand-held imaging probe benefits from the addition of a homogenous source distribution will ensure that the probe detects targets evenly along the length of the probe. A homogenous source distribution. i.e. all sources provide same intensity which ensures that there are no area's on the probe not getting illuminated. Reducing the likelihood of missing a target due to insufficient or uneven illumination

5) The hand-held imager was made to facilitate transition to the clinic specifically by simplifying positioning. The modifications will include an increase in portability such as using rack mountable or devices which can be bolted to racks.

6) The hand-held imager will feature a positional tracking system to determine the position of the probe during imaging. This tracking system must fit inside the portable rack. The initial work and eventual completion is the subject of another students Master's Thesis.

Table 6: The drawbacks of the Gen-1 optical imager and solutions on the instrumentation for the Gen-2 optical imager 


\subsection{Summary}

The drawbacks of the first Generation imaging system were addressed by the modifications mentioned in table 6 . In the materials and methods section of this proposal the instrumentation is listed and explained. The resulting system is labeled the Gen-2 hand-held optical imager. The system is portable, capable of $\mathrm{CW}$, transillumination via two probe head design and able to contour to tissues effectively with its 5 plate design. 
CHAPTER 3: Materials and Methods:

The Gen-1 hand-held optical imager has been previously described in chapter 2, section 2.8-2.11. Section 2.11 described the drawbacks of the Gen-1 hand-held optical imager along with several modifications (table 6). The advantages and disadvantages of the Gen-2 hand-held optical imager are related to the choice of material and the probe design. It should be noted that the Gen-1 was not very appropriate to fit curved tissue geometries with maximum contact, since it was developed primarily towards tissue phantom studies. The following sections describe the development of the main components of the instrumentation (sources, imaging probe, and detector) and the processing method employed to create the $2 \mathrm{D}$ surface plots while the experimental studies performed to date will be discussed in chapter 4-7.

\subsection{Instrumentation}

The instrumentation section below directly corresponds to specific task \# 1, which was to develop a Gen-2 hand-held optical imager. Figure 10 illustrates the instrumentation that was assembled within the portable enclosure. The Gen-2 hand-held optical imager includes light sources (provide illumination), hand-held probe (contacts imaging surface), and detector (measures optical signal). 


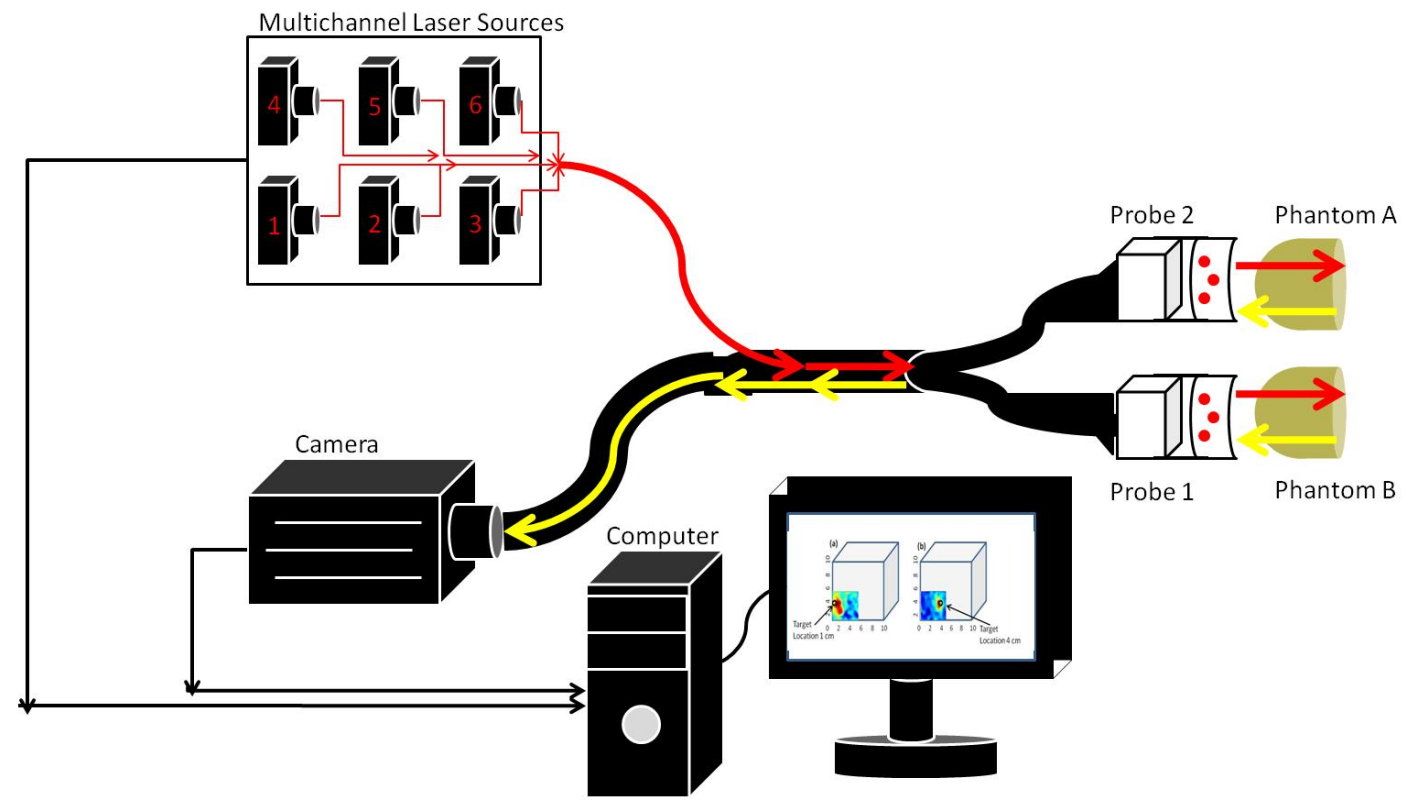

Figure 10: Schematic of the Gen-2 hand-held optical imager. The three main components of the imager are shown (source, detector and hand-held probes)

\subsubsection{Gen-2 sources:}

The Gen-2 sources are composed of two main components the multichannel controller and laser mount. The multichannel controller drives the laser mounts with an integrated laser diode driver and thermal electric cooler driver. The Gen-2 sources provide a homogenous stable distribution of monochromatic light onto the surface of the handheld optical imager. The laser system is composed of six independent diode laser mounts which are controlled by a multichannel controller and contain an input for frequency modulation (towards future modification of the system to operate in FD mode). The system provides a solution towards the demands of drawback number 4 in table 4 , which was that light from a single laser mount split into six optical fibers does not provide a homogenous source distribution. 


\subsubsection{Multichannel controller}

The Gen-2 hand-held optical imager's sources are driven and cooled by a Newport Irvine CA 92606, USA 8008 multichannel controller (Figure 11). The Newport 8008 features 8 independent channels and a large range of current values. These 8 independent channels were optimized individually to provide a homogenous source distribution. The probe has six sources leaving 2 channels for future expansion.

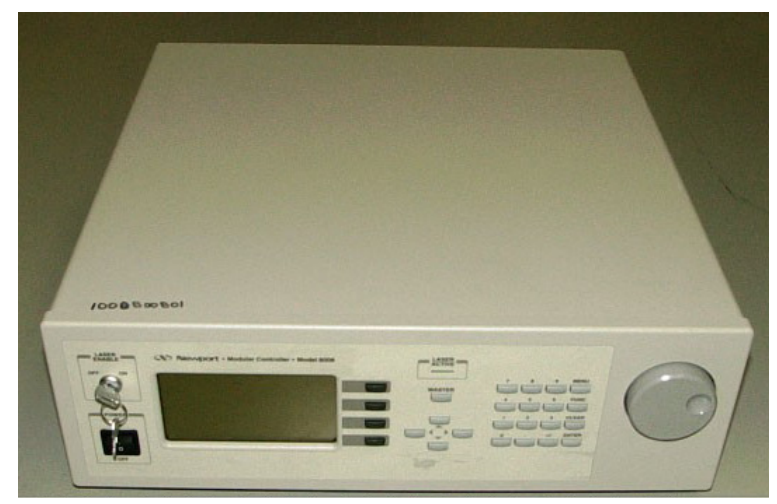

Figure 11: The source of the Gen-2 imager is driven by the multichannel controller that is a combination laser driver (LD) and thermoelectric cooler driver (TECD), which allows for the control of up to 8 independent laser mounts.

\subsubsection{Laser mount}

The TCLDM9 (Thorlabs, Newton, New Jersey, TCLDM9) is a sturdy temperature stable laser mount accepting a $785 \mathrm{~nm}$ laser diode and RF input for modulation. The diode employed (Thorlabs, Newton, New Jersey, 785P100) emits light at $785 \mathrm{~nm}$ and can produce up to $100 \mathrm{~mW}$ of optical power in CW (Figure 12). The laser mounts are used in conjunction with the parts below which serve as a coupling point with the optical fibers. A coupling point is a location where the laser diode emission and the optical fiber are attached on the same optical axis. The aspheric lens collimates the light from the laser diode onto the optical fiber and also maximizes the light entering into the optical fiber. Custom cables are required to operate the laser mount 
with a Newport 8008 multi-channel laser controller. In the appendix there is a table describing as to which pins are required to be connected.

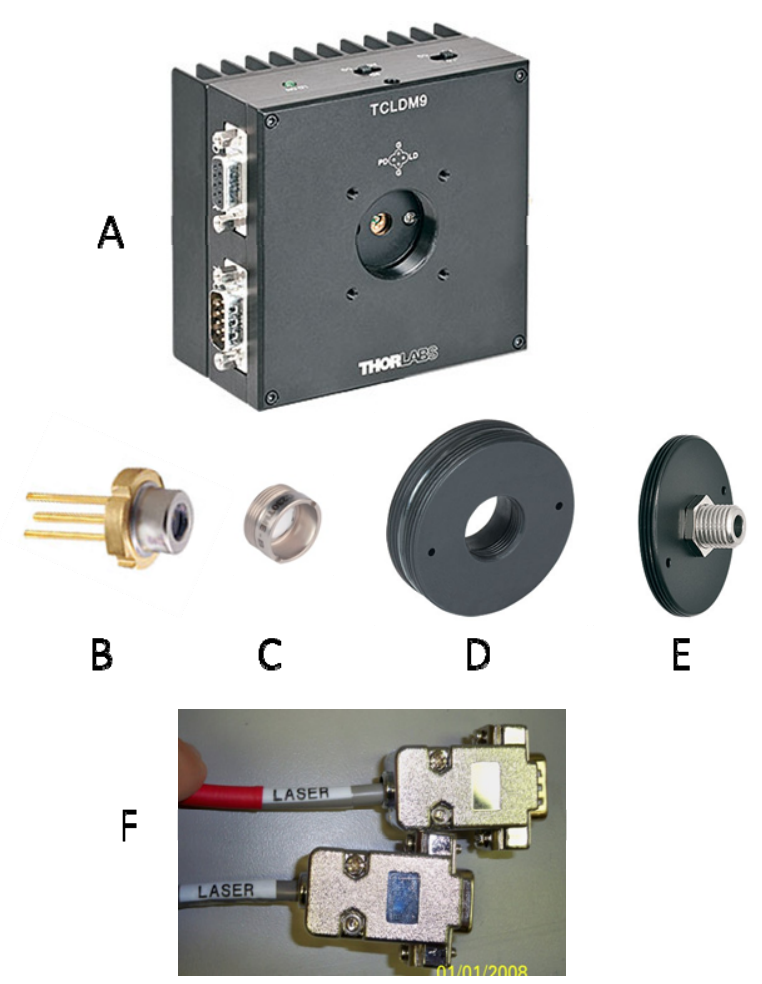

Figure 12: (A) laser mount TCLDM9,(B) laser diode 785P100,(C) C230TME-B - f=4.51 mm, NA=0.55, mounted Geltech aspheric Lens, AR: 600-1050 nm,(D) S1TM09 - SM1 to M9 x 0.5 lens cell adapter, (E) SM1SMA - SM1 (1.035"-40) to SMA fiber connector adapter plate,

The hardware components of the source system are the multichannel controller and the laser mount with added attachments. With these items chosen, the next section discusses optimization of the components to provide homogenous illumination. 


\subsubsection{Optimization of the source system}

Optimization of the source system i.e. ensuring homogenous and stable operation (minimal variability over time) is improvement $\# 4$ on table 6 , which is required for imager performance on target detectibility as will be discussed in chapter 4. Testing was performed using a Thorlabs, Newton, New Jersey P100, S121B an optical power meter with a detector bandwidth including $785 \mathrm{~nm}$ (see figure 13).

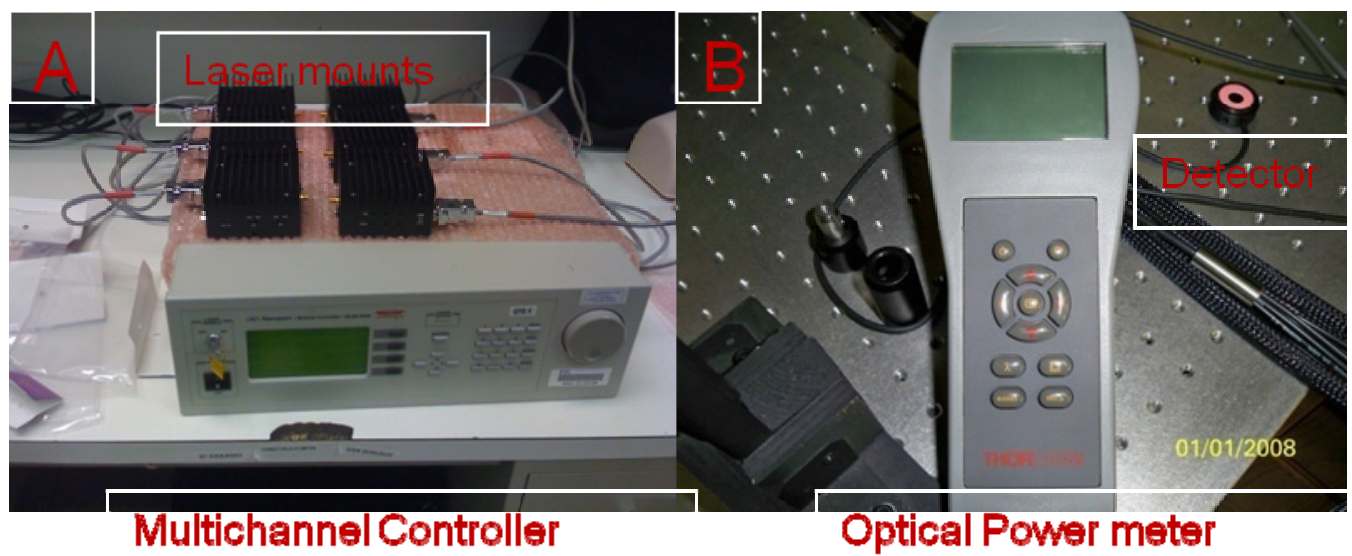

Figure 13: Multichannel controller and the laser mounts prior to stability testing with thermal electric driver active.

B) Optical power meter (can measure optical power from the laser mounts).

To ensure homogenous output of the laser investigation of the following topics was performed:

i) input currents vs. output optical power of the laser diodes; ii) stability in the temperatures over time.

The output optical powers from the laser diodes on their respective channels of the multichannel controller were recorded at different current values (Figure 14). The results were then used to find at which current a diode would provide a specific optical power. Each point on the graph is the average of 5 values taken after a 5 minute wait period for optimal stability. The current vs. optical power curves are different for every laser diode illustrating how diverse diodes from 
different silicon batches can be, and why six independent laser drivers are required to ensure homogenous output.

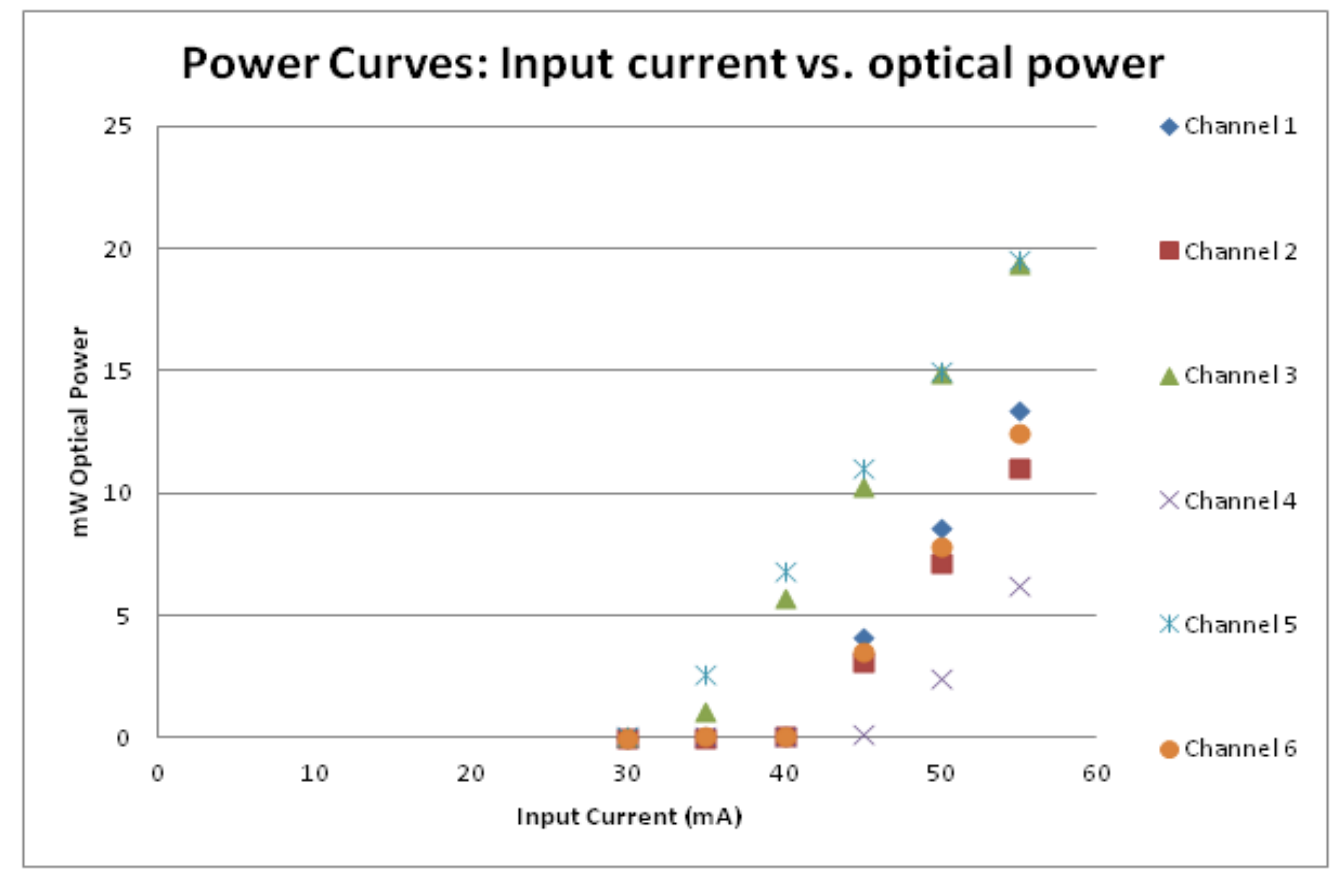

Figure 14: Input current vs. optical power for the six independent channels with their respective laser diodes

The power curve was then used to find the approximate value of current required for each laser diode channel pair to output $4 \mathrm{~mW}$ of optical power. The purpose was to establish a baseline output power from which temperature fluctuations could be observed. It had been observed that temperature fluctuations impacted optical power output creating non-homogenous illumination, hence reducing the variation in temperature would also reduce the variation on output optical power of the laser mounts.

To address the heat buildup an extractor fan was added to each mount and stability was tested after a 30 minute wait period. An optical power of $4 \mathrm{~mW}$ was chosen to test the cooling solution as it was close to the values of 2 and $5 \mathrm{~mW}$ that would have been used on experimental studies. Output powers were recorded over a 150 minute period to quantify the fluctuation on optical 
power. The mean and standard deviation without a fan was $(3.7 \mathrm{~mW} \pm 0.26 \mathrm{~mW})$, while with a fan the values were $(4.06 \mathrm{~mW} \pm 0.17 \mathrm{~mW})$. A t-Test: (Paired Two Sample for Means) was used to determine if the addition of the fan made a significant difference. The $p$-value found was 0.0002 and hence there is a significant difference between the removal of heat between the two designs tested.

Due to the significant increase in homogeneity of output light $(\mathrm{mW})$, the addition of extractor fans for each mount was implemented. The power supply for the fans is an Athena micro ATX (Athena power City of Industry, CA) and the fans employed are $90 \mathrm{~mm}$ (Masscool, City of Industry, CA). Figure 15 shows the final rack mountable design. The arrangement chosen provided maximum airflow while allowing a user to access to locations to connect cables for optical fibers, laser driver power, thermoelectric cooler power and RF input signal.

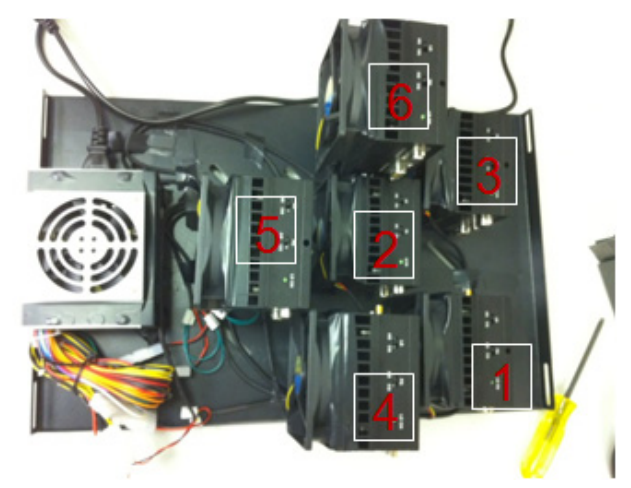

Figure 15: The final configuration of the laser mounts, on a rack mountable stage. The mounts are labeled with their channel number (1-6)

The next section employs the GPIB connectivity of the multichannel controller to provide automated control to the laser mounts, allowing for operation of the Gen-2 imager from a single software. 


\subsubsection{Computer controlled multichannel controller.}

The multichannel controller manual and automated Labview controls (Figure 16) were determined to be slow to activate the system and difficult to operate. To reduce the number of steps required for operation, (such as setting each LD or TEC independently- increasing complexity and time) automated controls via a Labview program (Figure 17) were generated by modifying an existing Newport supplied automation control. The implemented features were (i) individually or sequentially turn on and set current values for each laser mount (ii) software limited safety feature allowing for a maximum of $70 \mathrm{~mA}$ from the LD (iii); and reduced the time from manual operation of the system (from $50 \mathrm{~s}$ to $15 \mathrm{~s}$ ). 


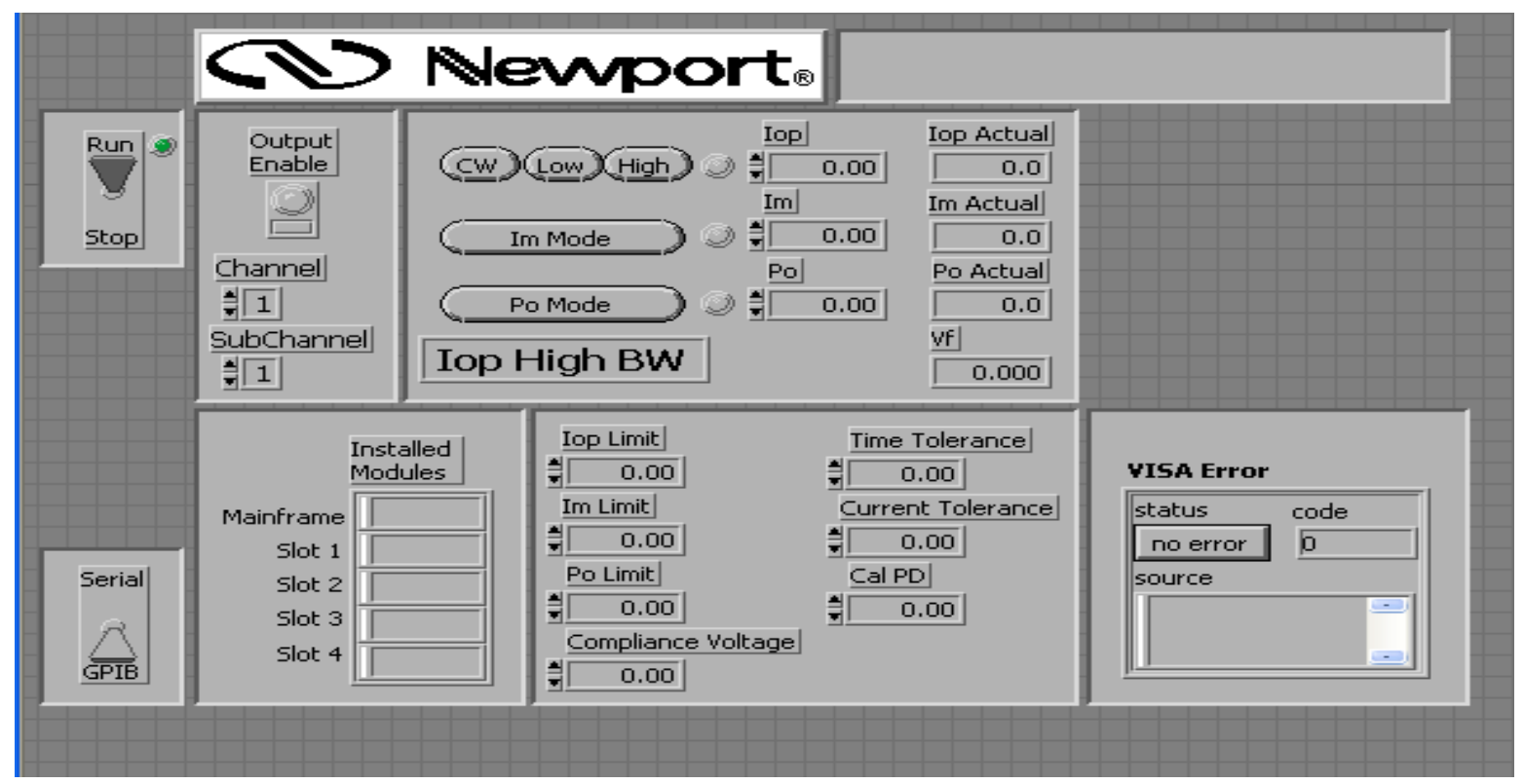

Figure 16: Newport 8008 Labview driver computer control

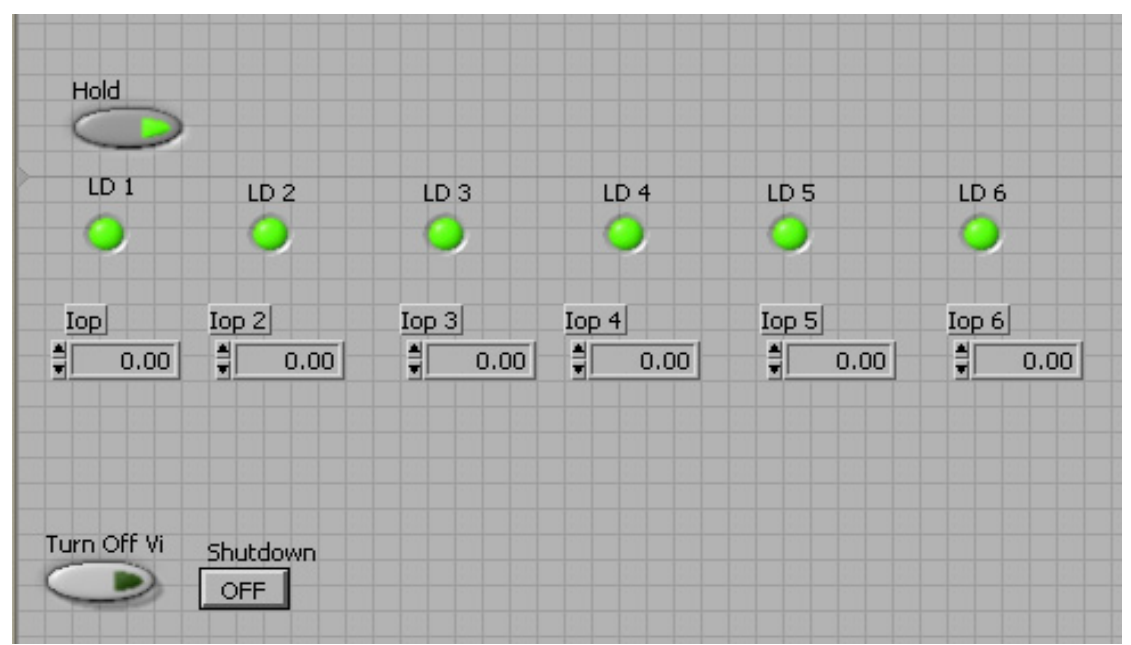

Figure 17: Final Automation result (Front Panel) of the computer controlled multichannel controller

With the hardware and software of the source system completed the hand-held imager which contacts the tissue surface is discussed in the next section. 


\subsubsection{Gen-2 probe construction.}

The Gen-2 probe is a forked hand-held optical imaging probe composed of an imaging surface or probe face which contains optical source and detection fibers. The optical source fibers are connected to the laser mount discussed previously, while the optical detection fibers travel from the probe face to the detector where they are bundled together.

(Table 4 (i) The Gen-2 hand-held imaging probe was reduced in size and possesses improved flexibility (ii) The Gen-2 hand-held imaging probe features a forked design to allow for bilateral simultaneous as well as transillumination imaging (iii) The Gen-2 hand-held imaging probe was produced from acrylic and silicon not aluminum (patient discomfort by reducing heat transfer). The Gen-2 handheld probe has 192 detection fibers and 6 source fibers placed on the imaging face. The probes are split into two sections $4 \times 5 \mathrm{~cm}^{2}$ containing 96 detection fibers and 3 sources fibers (Polymicro Technologies Phoenix, Arizona). The following sections will discuss the source fibers, detection fibers and the probe face.

\subsubsection{Source fibers}

The source fibers were cut to a length of 2 meters and polished with different grades of "sandpaper". The polishing process is included in the appendix. One end of the source optical fibers was attached to the probe face while the other was fitted with an SMA connector and attaches to the laser mount. The source distributions for the first and Gen-2 hand-held optical imager are shown in Figures 18. The total area of the two probe heads (each probe head is $4 \times 5$ $\mathrm{cm}^{2}$ Gen-2 hand-held optical imager) is similar to the total probe head area of Gen-1 hand-held optical imager design $\left(4.5 \times 9.5 \mathrm{~cm}^{2}\right)$. Hence, the source fiber layout (i.e. 6 sources on the Gen-1 hand-held optical imaging probe) was maintained for the Gen-2 hand-held optical imaging probe ( 3 sources on each of the two probe heads). The sources are arranged to maximize the total area illumination of the Gen-2 probe $[29,30,38,39]$. The optical source intensity distribution of the 
Gen-1 and Gen-2 optical fibers were compared with an optical power meter (Thorlabs, Newton, New Jersey P100, S121B) after optimization of the source output to $2 \mathrm{~mW}$. The Gen-1 handheld optical imager's source distribution varied widely, with the strongest source at $2.1 \mathrm{~mW}$ and the weakest at $0.54 \mathrm{~mW}$ with a mean and standard deviation of $(1.02 \pm 0.937) \mathrm{mW}$, respectively. The Gen-2 hand-held optical imager through its independent laser sources had a mean and standard deviation of $(2.05 \pm 0.060) \mathrm{mW}$.

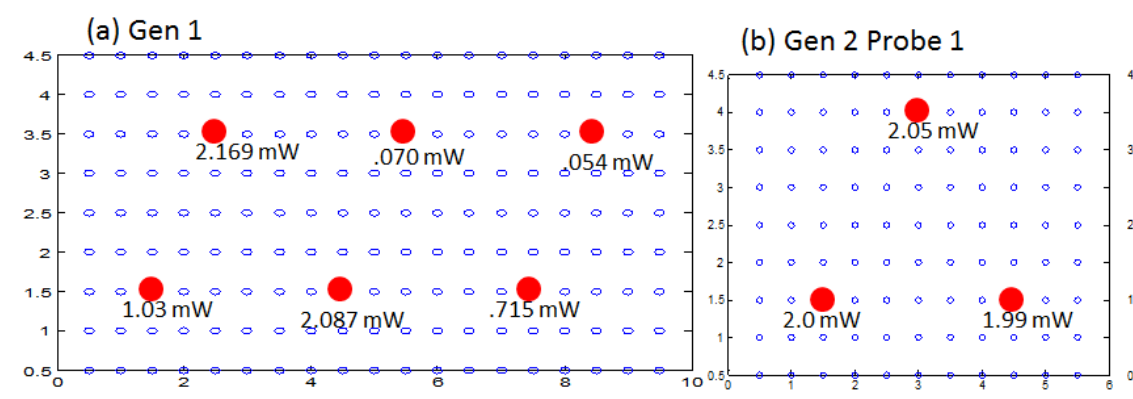

(c) Gen 2 Probe 2

Figure 18: The source detector layout of the hand-held optical imagers (a) Gen-1 (source mean and std $1.02 \pm 0.937$ $\mathrm{mW}$ ) (b) Gen-2 (source mean and std $2.05 \pm 0.060 \mathrm{~mW}$ )

The next section discusses the optical detection fibers which collect the optical signal from the tissue after it has been illuminated by the source.

\subsubsection{Detection fibers}

The same polishing process done for the source fibers was also done for the detection fibers. The optical detection fibers were to be bundled together into a camera adaptor piece previously produced by a senior design team and modified by laboratory members (Figure 19). 


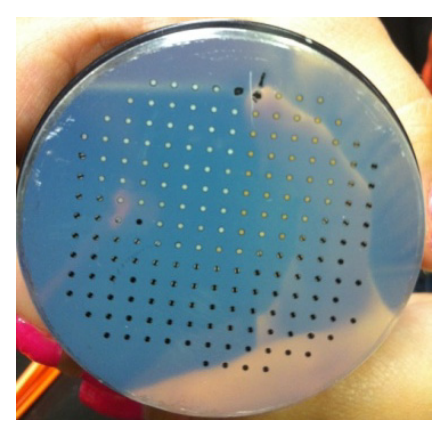

Figure 19: Optical detection fiber bundle of the Gen-2 imaging probe.

The camera adaptor part has 192 fibers holes drilled in $0.25 \mathrm{~cm}$ spacing. The adaptor fits into lens array and the lenses focus the image onto the ICCD detector. The bundle has a diameter of $4 \mathrm{~cm}$. The fibers were numbered and correspond to numbers on the probe face. A soft cloth and sleeve was placed over the optical fibers to prevent scratch damage along the length. The optical detection fibers had one end glued to the optical bundle while the other end was positioned on the probe faces. Polishing the surface of the camera adaptor piece was contracted out to Romack (Williamsburg, VA) that had equipment better suited for what was required. The task involved cutting and polishing the surface of the camera adaptor piece without disturbing the optical fibers on the Gen-2 hand-held optical imaging probe.

\subsubsection{Optical probe face}

The imaging face or probe is the part of the equipment that makes contact with the tissue surface. The Gen-2 hand-held optical imaging probe was designed as a forked probe allowing for simultaneous bilateral and transillumination imaging. The Gen-2 hand-held optical imaging probe is constructed out of a plastic (acrylic) and a silicon mix. The plastic backing provides rigidity, while the silicon adds flexibility to the Gen-2 hand-held imager. The fibers are threaded through the holes on the optical probe surface and are fixed flush to the imaging surface. Figure 20 below displays the initial plastic backing of the probe with the pre cut holes for the fibers. The 
Gen-2 hand-held optical imaging probe was design to be capable of flexing which enhances the surface contact when compared with the previous Gen-1 hand-held optical imaging probe. A 5part probe head with flexible silicone interlocking allows the Gen-2 probe to contour to breast curvatures (Figure 20) [37,38, 39]. 


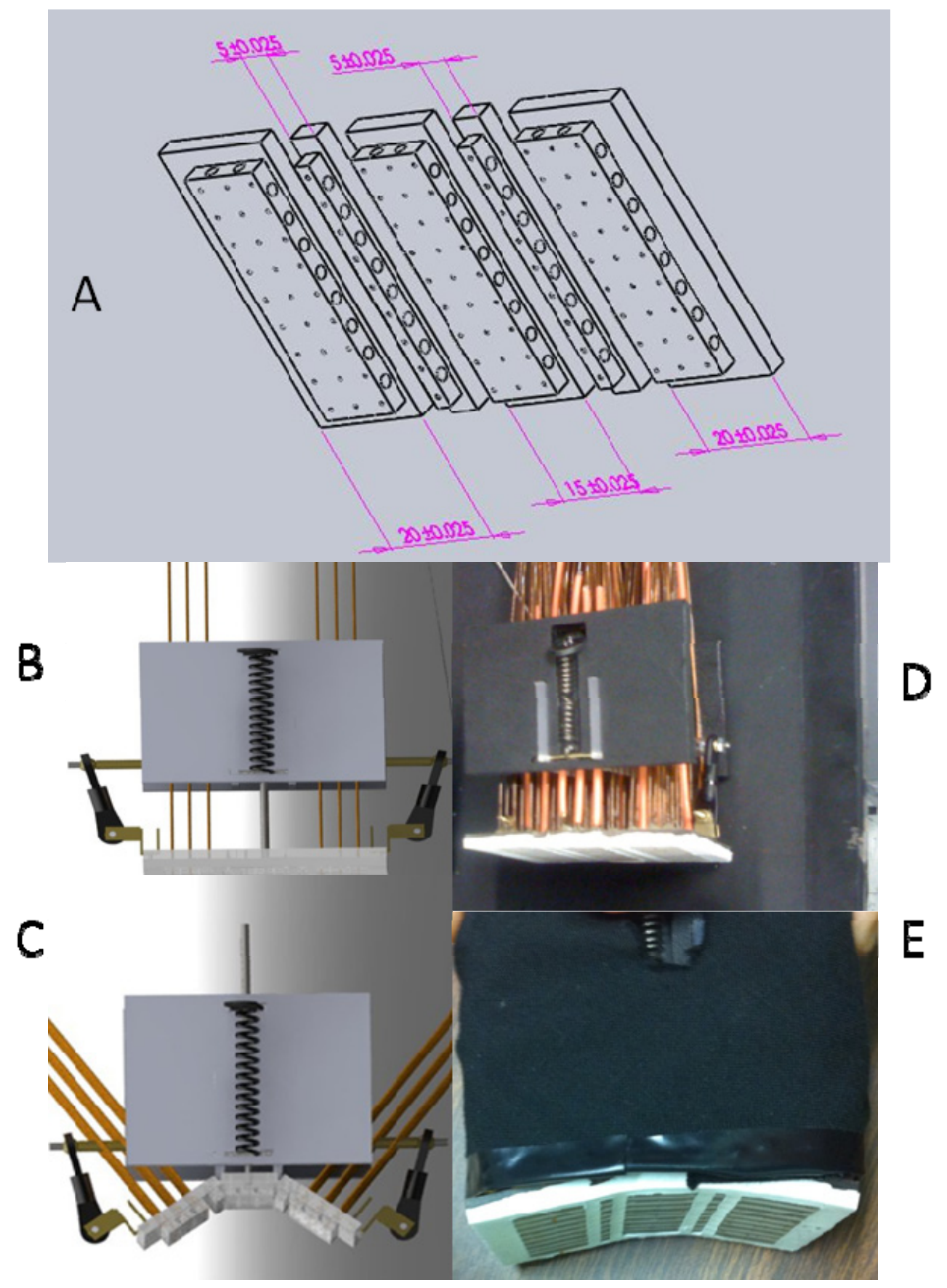

Figure 20: (A) Gen-2 hand-held probe face (5 part design). (B) Model of the Gen-2 hand-held probe flat (aerial view), (C) Model of the Gen-2 hand-held probe curved (aerial view), (D) The Gen-2 hand-held probe flat, (E) The Gen-2 hand-held probe curved 


\subsubsection{Probe surface contact}

Experimental measurements of surface area contact with the Gen-1 and Gen-2 probe heads was determined to be $\sim 30 \%, 86 \%$ respectively. The experiments were performed using a $13 \mathrm{~cm}$ diameter solid cylindrical phantom. The probes were adjusted to their maximum allowable curvature as appropriate for the cylindrical phantom and placed in contact to its cylindrical surface. A white light digital image of the set-up was acquired from the top view (see Figure 21).

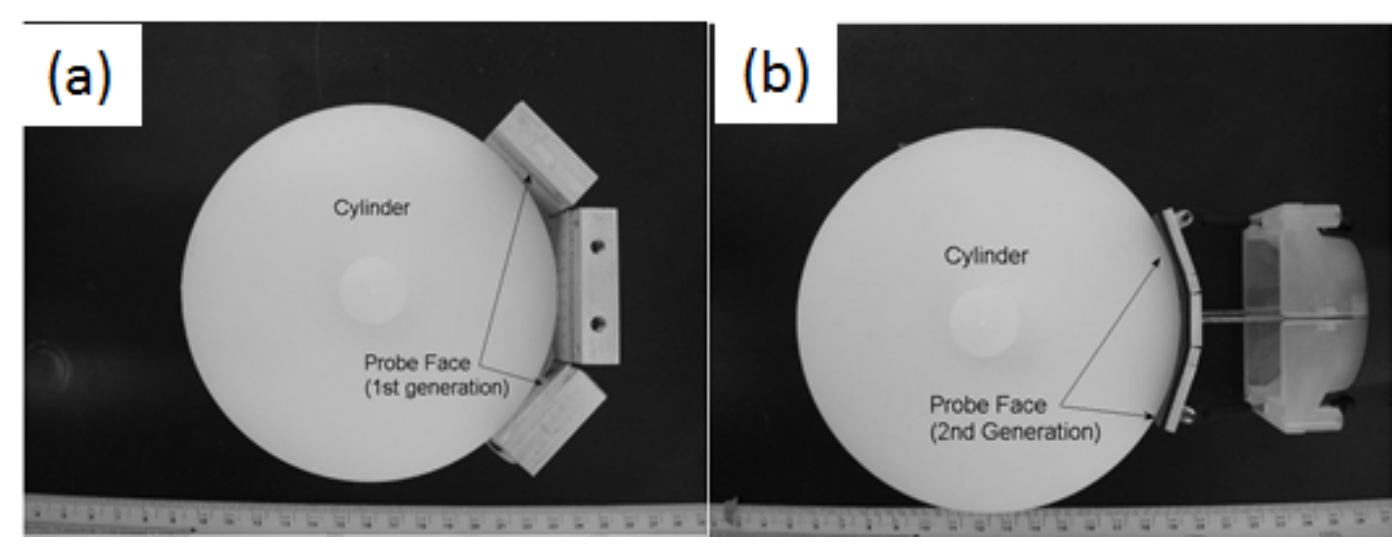

Figure 21: The experimental measurements of curvature for the Gen-1 (a) and 2 (b) hand-held optical probes resulted in values of $\sim 30,86 \%$ using a $13 \mathrm{~cm}$ solid cylindrical phantom respectively.

The digitized image was converted to grayscale and a Sobel filter was applied in order to display only the edge information. Three points (or pixel locations) on each large probe plate (i.e. 3 probe plates each for Gen-1 and Gen-2's single probe head) was determined and fitted to a circle. The curvature $\left(C_{\text {probe }}=1 / R\right)$ of each fitted circle is estimated from the radius of the circle (R), and the surface contact area $\left(A_{\text {contact }}\right)$ for a single probe plate is estimated using the equation below.

$$
A_{\text {contact }}=\frac{C_{\text {probe }}}{C_{\text {phantom }}} \times A_{\text {probe }}
$$


where $C_{\text {probe }}$ and $C_{\text {phantom }}$ are the radius of curvatures of the each large probe plate and cylindrical phantom (in $\mathrm{cm}$ ), respectively. The cylindrical phantom's curvature $C_{\text {phantom }}$ (i.e. $1 / R_{\text {phantom }}$ ) is $0.154 \mathrm{~cm}^{-1}$, based on its $6.5 \mathrm{~cm}$ radius $\left(\mathrm{R}_{\text {phantom }}\right) . A_{\text {probe }}$ is the surface area of the respective probe plate that is imaged $\left(\mathrm{cm}^{2}\right)$. The total surface area contact is the summation of three probe plate's contact area (for Gen-1 and Gen-2 probes).

From this experimental approach, the total surface contact areas for Gen- 1 and Gen-2 probes are $\sim 30 \%$ and $\sim 86 \%$, respectively. In additional to the above procedure, a template of the Gen-1 and Gen-2 probes was placed on an ink pad, then placed on a $13 \mathrm{~cm}$ cylinder which contained a sheet of paper wrapped around its circumference. The resulting ink blot was measured with Vernier calipers. The surface contact was calculated by dividing the area of the ink blot by the total surface contact area of the probe (equation 18).

$$
\text { Surface contact }(\%)=\frac{\text { Area of ink blot }}{\text { Total area of the probe }}
$$

This approach also provided similar values of effective surface contact area (i.e. $\sim 26 \%$ for Gen-1 and $\sim 84 \%$ for Gen-2 probe, respectively). On an average, the Gen-2 probe has almost 3 times greater surface contact on a cylindrical phantom over the Gen-1 probe. Effective surface contact area was not obtained from human breast tissues, since the probe contact can change on the nonconforming soft tissues. This can cause inconsistency in the experimental data based on pressure applied onto the tissue. Although, the above study on a cylindrical phantom clearly demonstrates that Gen-2 probe has an improved surface contact over Gen-1 probe.

\subsubsection{Detector}

The detector is composed of the PI-Max II produced by Princeton Instruments (Figure 22) and the series of lenses, lens tube and a ring attachment for the optical fiber bundle. The detector is CCD based, since collection of light signal from the 196 optical detection fibers (probe contains 
196 optical detection fibers) is important due to the probe face design (large surface area imaging). The PI-Max II produced by Princeton Instruments (Figure 22) offers a built in intensifier which can be software controlled via Labview or Winview. The ICCD camera will be able to collect the signal intensity (in a.b.u.) on the fiber bundle and save the image as a file which allows access to the data. The probe fibers are attached to the camera via a series of lens tubes. The lens tubes or assembly are coupled to the ICCD camera via a Nikon focusing lens (Nikon Melville, NY US branch, Nikon $50 \mathrm{~mm} \mathrm{f} / 1.8 \mathrm{D}$ AF) that is C-mounted to the ICCD camera.

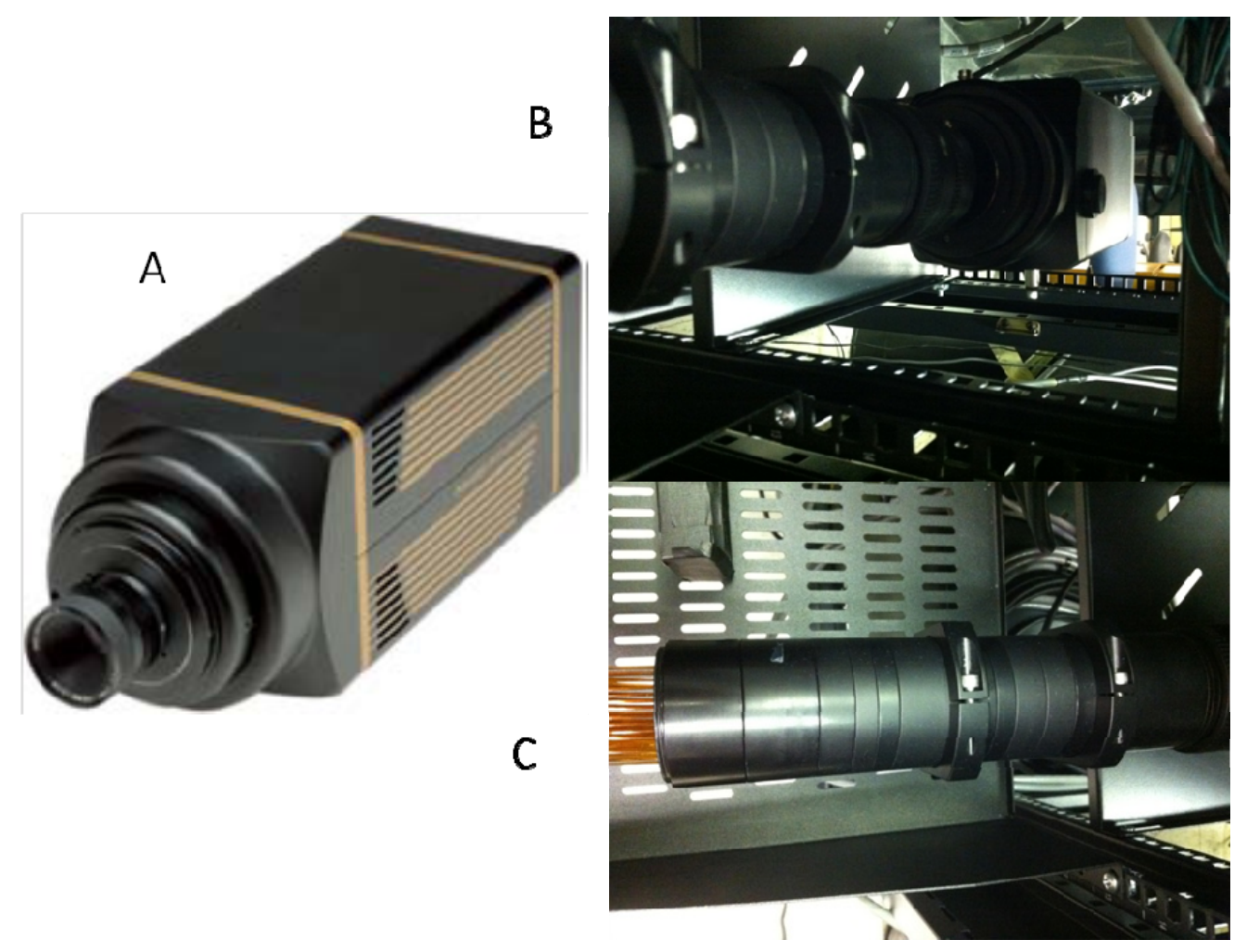

Figure 22: (A)The PI-Max II is a ICCD camera produced by Princeton instruments towards the detection of low intensity light, (B) the Gen-2 camera within the enclosure for the hand-held optical imager, (C) Optics employed in conjunction with PI-Max II, such as lens tubes and other lens adapters 
The lens assembly for the Gen-2 hand-held optical imager focuses the image of the optical fiber bundle onto the ICCD of the camera (Figure 22). The distance between the camera's intensifier and the optical detection fiber bundles were adjusted based on equation (19) thin lens equation

$$
\frac{1}{S_{1}}+\frac{1}{S_{2}}=\frac{1}{f}
$$

where the focus of the lens $f, S_{1}$ is the distance from the lens to the hand-held probe and $S_{2}$ is the distance from the lens to the image. The equation could be solved for either $S_{1}(67 \mathrm{~mm})$ or $S_{2}$ $(106 \mathrm{~mm})$. However the distance between the surface of the camera and the image intensifier cannot be changed in the Gen-2 hand-held optical imager and must be added to $S_{2}$.

\subsubsection{Portable rack mounting}

The portable enclosure allows for portability while protecting and facilitating use of the current and future components of the Gen-2 imager (figure 23). The portable enclosure addresses advantage 5 on table 6 (i.e. portability) and with the inclusion of the system of a portable cart the $\mathrm{CW}$ component of the Gen-2 handheld imager is complete. The portable enclosure (Bud industries Willoughby, $\mathrm{OH}$ ) is a rack mounting system allowing for the placement of parts to be optimized to reduce heat by placing high heat generating equipment upwards near exhaust fans. 


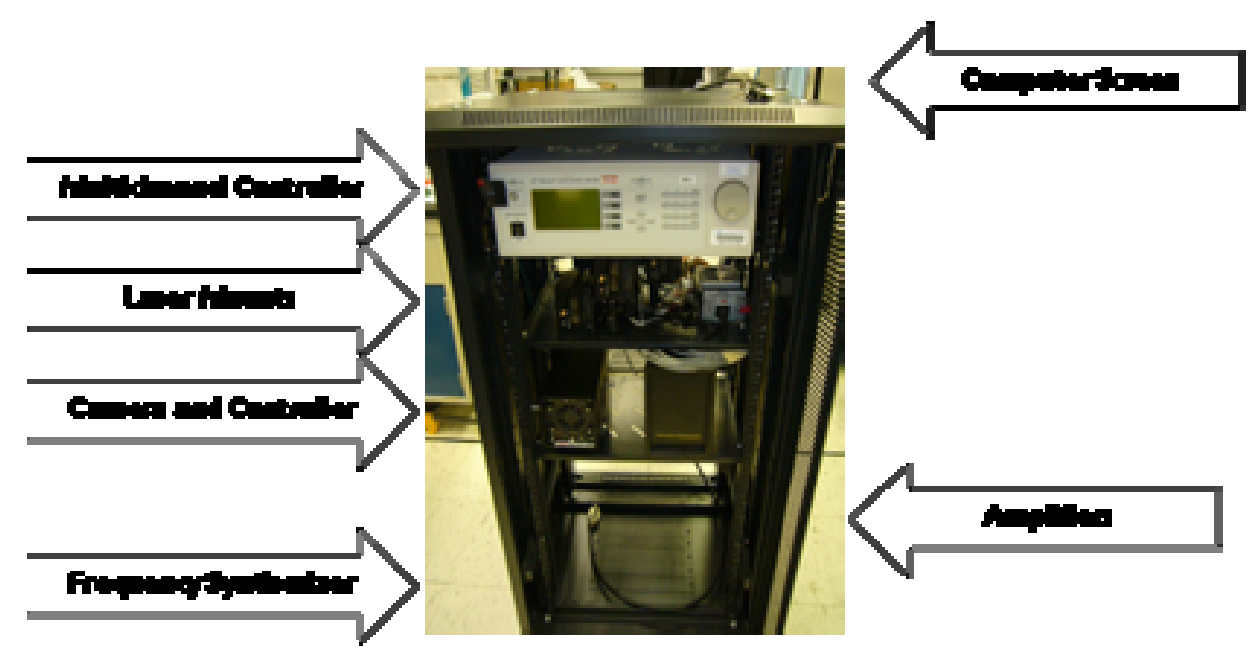

Figure 23: The Gen-2 hand-held optical imager is constructed inside of a portable cart (labels indicate the location of hardware).

The system above is an efficient portable rack that can keep the components of the Gen-2 handheld optical imager under appropriate temperatures for improved image quality. The next section will discuss the component selection of how the component selection found on the Gen-2 hand-held optical imager operates (Principle of Operation).

\subsubsection{Principle of operation}

The probe face composed of 192 optical detection fibers and six source fibers placed upon the surface to be imaged. The source fibers relay the light at a wavelength of $785 \mathrm{~nm}$ from the laser mounts powered by the multichannel controller. The source system is operated at a $\mathrm{CW}$ mode while providing a homogenous illumination upon the imaging surface. The light from the sources illuminates the tissue and propagates through the tissue. A small portion of the light returns to the probe surface as specular reflection but the light is not collected by the detectors. The majority of the light propagates through the tissue where it is attenuated and scattered due to interactions with scattering or absorbing molecules such as hemoglobin (absorbers) and lipids 
(scatterers). In the case of reflectance imaging the optical detection fibers on the probe collect the diffuse reflection signal. The collected signal travels back via the optical detection fiber bundle. The signal from the detector fiber bundle is focused on to the ICCD camera. Where the light signal hits a photocathode generating a voltage signal. The voltage signal is amplified (Micro channel plate) and accelerated towards the phosphor screen which converts the electrons to photons. The optical intensity signals from the optical fiber is collected via the ICCD and is then processed in the computer to generate a 2D surface contour plot of the intensity distribution of the imaged surface. The processing required to produce the final version of the $2 \mathrm{D}$ surface contour plot will be discussed in the next section.

\subsection{Data Processing and Image Analysis}

The Gen-2 hand-held optical imager was developed towards visualization of optical tissue contrast (Generates images) with a focus on target (tumors when referring to cancer) detection. To date the Gen-2 system depends on endogenous tissue contrast created by the pooling of blood in an area as CW measurements can create a 2-D contour plot of areas of high and low absorption. The image processing involves (1) collection of optical signal, (2) averaging of the collected optical signal, (3) extraction of the optical signal, (4) normalization to account for instrument effects, (5) subtraction to enhance contrast, (6) removal of detectors $0.5 \mathrm{~cm}$ around source, (7) normalization, and (8) polynomial fitting (as shown in Figure 24). 


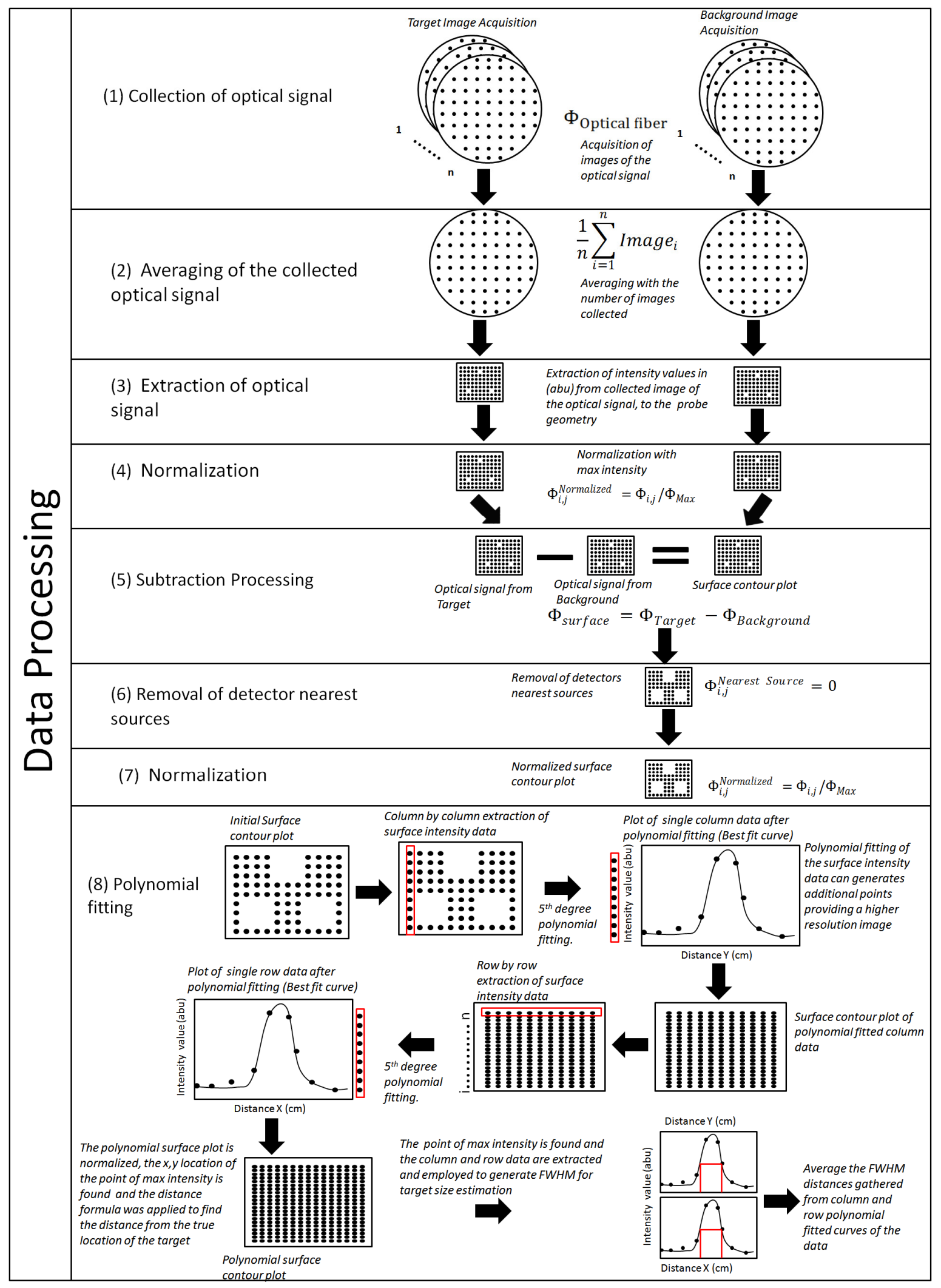

Figure 24: Flowchart of the steps of data processing. 


\subsection{1 (1) Collection of optical signal}

The collection of optical signal involves the acquisition of the target and background optical data. In this step the camera controls such as exposure time, and the number of repeated images captured is set. The optical signal is collected with an ICCD camera in terms of a.b.u., which corresponds to the detected intensity signal from the various detection points on the probe surface.

\subsection{2 (2) Averaging of the collected optical signal}

The collected optical signal is then averaged across the number of repeated images captured, generating a single image from which the optical intensity measurements from the probe surface will be extracted.

\subsection{3 (3) Extraction and placement of optical intensity measurement.}

Initially, an alignment between the location of the detectors in the acquired image and detector placement image must be performed. The detector placement image has the locations of each of the optical fibers and must be made to match the scale and rotation of the acquired image. The rotation requires 2 calibration points with the respective $x, y$ values (2 pixels) to find the angle of rotation $(\theta)$ and scaling factor (calibration factor for image size as seen on the detector) (as shown in Figure 25). The alignment allows for extraction of the detected intensity signal w.r.t to the detector fiber location on the circular mount imaged by the camera. This is followed by projection of the detected intensity signal (corresponding to each detector fiber) onto its respective $x-y$ locations on the imaging surface (or the probe face). In Figure 25 the rotation $(\theta)$ would be angle associated between detector 1 and 161 . 


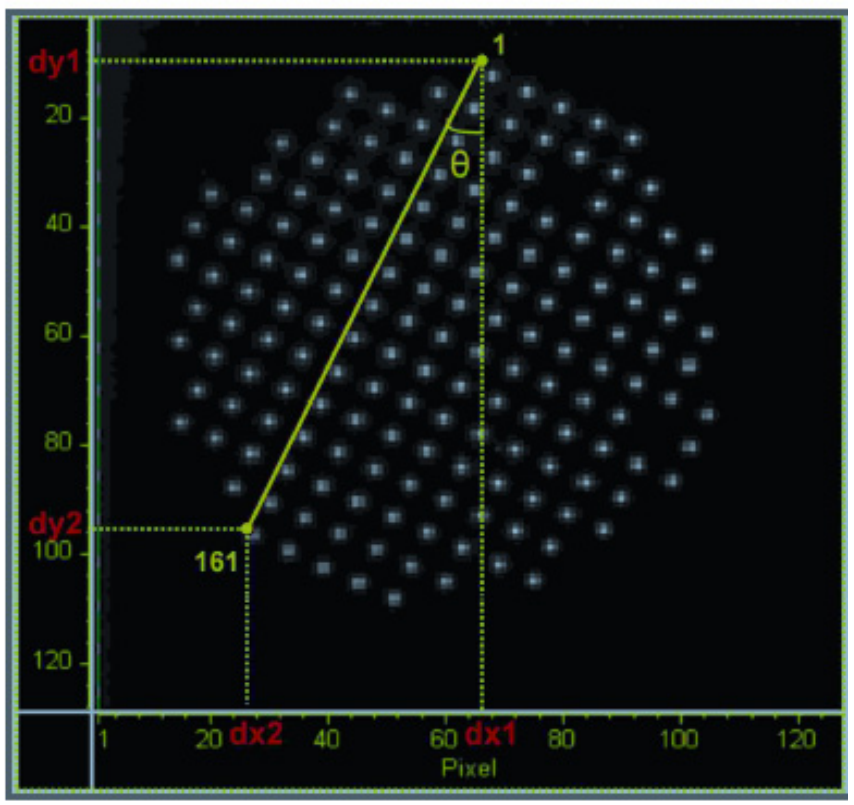

Figure 25: Illuminated optical fiber bundle of the Gen-2 imager as captured via the ICCD (detector). The points selected are calibration points required to match the location of the optical fiber on the fiber bundle with the corresponding end on the probe face.

\subsection{4 (4) Normalization}

The data is normalized with regards to the max intensity of each probe's averaged intensity image (for a given experimental case). Normalization is performed in order to eliminate instrument effects arising from detector in-homogeneities and source fluctuations. The current normalization approach relies on the assumption that all the detector fibers connected to a given probe head are of equal length, and are thus not the cause for variations in the intensities across the different detector fibers. 


\subsection{5 (5) Subtraction Technique}

In all experimental cases, a subtraction technique is employed to remove the background noise and improve the optical contrast between the target(s) and the background (figure $26 \mathrm{~A}, \mathrm{~B}$ ) (True target location is represented by a black circle). The figure displays 2D surface plots of the total processing performed on 1 experimental case. A) presents the unsubtracted image while B) the subtracted image. In the unsubtracted image there is not enough contrast between the absorption and the total optical signal collected by the Gen-2 imager. (C,D are removal of detectors and polynomial fitted data are explained in later sections).

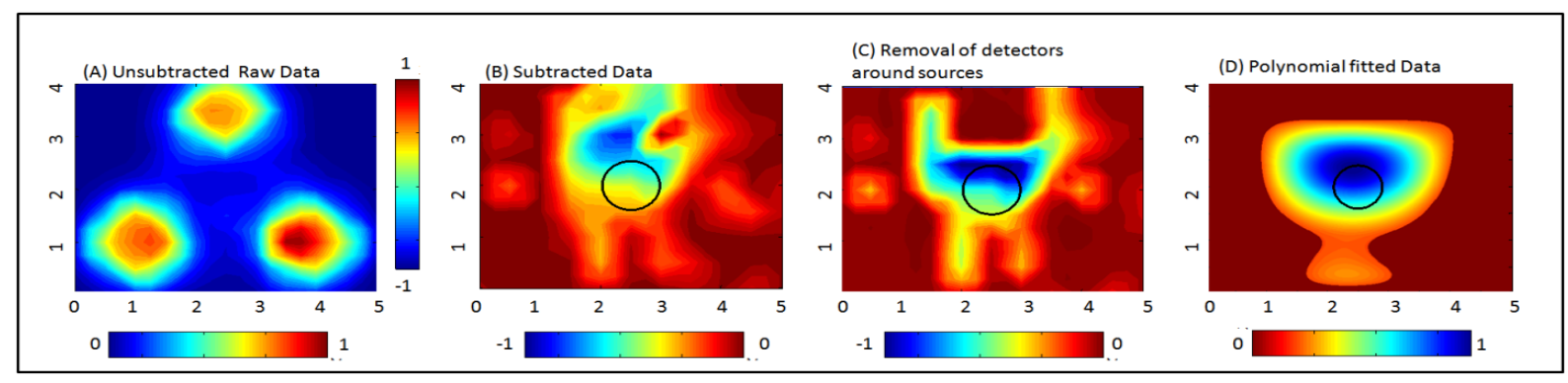

Figure 26: 2D surface contour plots of a $0.45 \mathrm{cc}$ spherical target placed inside of a liquid liposyn phantom generated through the various steps of the data processing procedure. (A)The unsubtracted (A) and subtracted (B), (C) Subtracted data with the detectors nearest the sources removed and (D) polynomial fitted data of subtracted data.

For reflectance imaging studies three possibilities could occur i) Target removal with a single phantom (as explained above for transillumination), ii) simultaneous imaging, and iii) sequential imaging. For transillumination imaging studies the forked probe design is placed on a slab phantom $5.5 \times 5.5 \times 10 \mathrm{~cm}^{3}$ where each probe is oriented on opposite sides, however only a single probe is employed as a detector where the background is acquired via target removal. The next sections will explain the two distinct methods of subtraction, which is target removal employed for single probe reflectance and transillumination and bilateral subtraction (dual probe imagingreflectance). 


\subsubsection{Target Removal single probe detection reflectance and transillumination:}

Acquisition involves placing and removing the target as required for subtraction i.e. (target background) (figure 27).

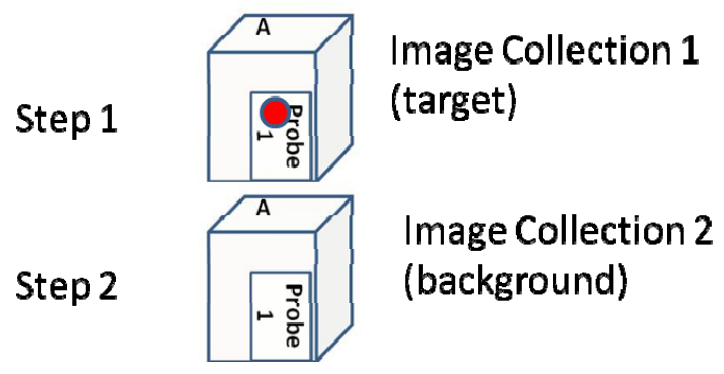

\section{Step 3 Subtract image 1(target) and 2 (background)}

Figure 27: Subtraction is an optical processing technique to enhance image contrast that centers around the removal of the target to generate a difference between the target (step 1) and background (step 2) images.

\subsubsection{Bilateral imaging (Simultaneous and Sequential):}

Bilateral imaging (simultaneous and sequential) requires two identical phantoms, one with a target and another without. Subtraction technique is performed by subtraction of the normalized averaged intensity data obtained from the phantoms with and without targets i.e. (target background). A second normalization is performed after subtraction, where the subtracted signals at each detector point are normalized w.r.t the maximum subtracted intensity for the given probe image (and the experimental case).

Bilateral simultaneous imaging involves the acquisition of the optical signal from two independent phantoms with the dual probe design and subtracting the two images (one image from each probe), (Figure 28). Bilateral simultaneous imaging could be employed in cases where the target is not removable. Using two phantoms one with a target and the other without it 
mirrors a patient with unilateral breast cancer. The technique depends on being able to generate a consistent pattern image relating the two probes.

- (Step 1) Place probe 1 on phantom A and probe 2 on phantom B and collect an image

- (Step 2) Place probe 2 on phantom A and probe 1 on phantom B and collect an image

- (Step 3) Subtraction of the image of probe 1 on phantom A from probe 2 on phantom B yields the variability within the phantoms plus the variability of the probes.

- (Step 4) Subtraction of the image of probe 1 on phantom A from probe 2 on phantom A yields the variability within the probes. The variability between the two probes is a pattern image relating the two probes.

- (Step 5) Subtract the image result of Step 3 from the image result of Step 4. The new image is the variability of the phantoms minus the variability of the probes and hence the real target image.

Bilateral simultaneous imaging has the potential to be a single scan imaging technique if a consistent pattern or related image (variability between the probes) could be developed between the two probes; thus reducing the placement error, imaging time and also increasing image quality.

Bilateral sequential imaging involves the acquisition of the optical signal from two independent phantoms, then switching the position of the probes and re-acquiring the optical signal. The two images are then subtracted (Figure 28). Bilateral sequential imaging could be employed when the target is not removable.

- (Step 1) Place probe 1 on phantom A and probe 2 on phantom B and collect an image - (Step 2) Place probe 2 on phantom A and probe 1 on phantom B and collect an image

- (Step 6) Subtraction of the image of probe 1 on phantom A from probe 1 on phantom B yields only variability within the phantoms and hence the target image. 


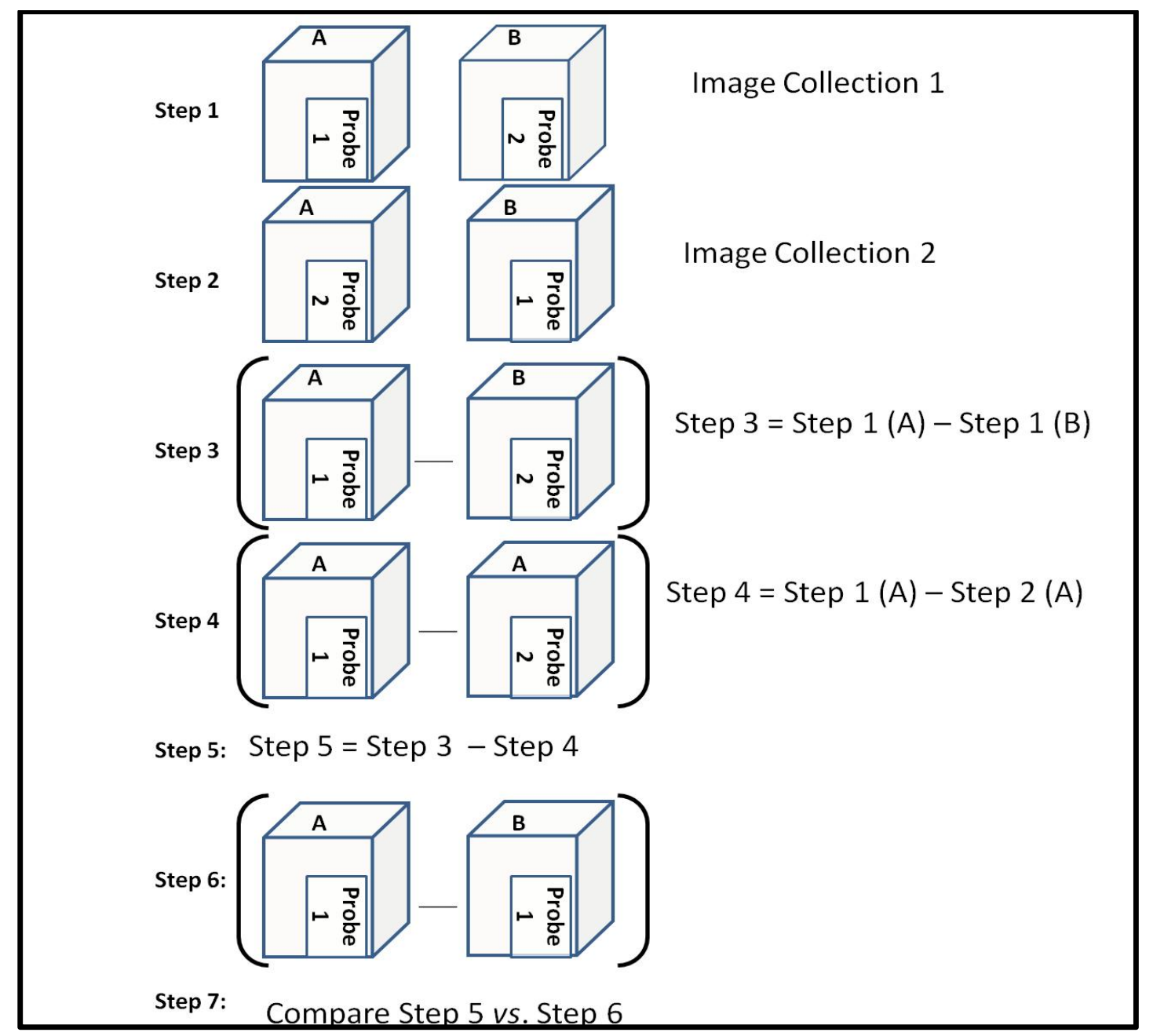

Figure 28: Data processing for bilateral imaging. Simultaneous and sequential bilateral imaging apply subtraction processing using the contralateral breast as a background.

\subsection{6 (6) Removal of detectors nearest sources}

Despite the application of the subtraction technique, artifacts typically exist at the detection points close to the points of illumination, say $\sim 0.5 \mathrm{~cm}$. Hence to remove any residual excitation leakage of the sources close to the detection points, the detection points located $0.5 \mathrm{~cm}$ around the points of illumination was removed (Figure 29). A comparison of figure 26 subtracted (B) and subtracted with detectors removed $(\mathrm{C})$ visually suggests that the localization of the target would be enhanced with the removal of the detectors nearest the points of illumination. 

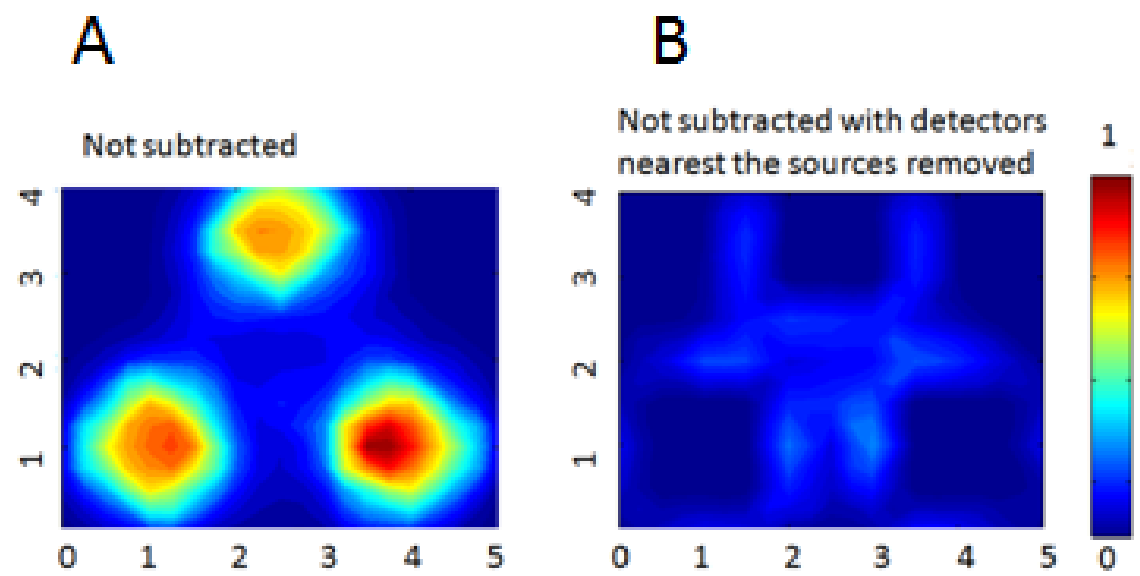

Figure 29: (A) 2D surface contour plots of the excitation leakage of the Gen-2 hand-held optical imager. (B) Removal of the detectors nearest the points of illumination $\sim 0.5 \mathrm{~cm}$ reduces the excitation leakage from the sources to the detector points and increases homogeneity of the points submitted to the next processing step polynomial fitting.

\subsubsection{Normalization:}

Normalization is performed again to retain a scale that ranges from 1 to 0 .

\subsection{8 (8) Polynomial fitting}

Previously surface contour plots such as (A), (B), (C) on figure 26 depended on linear interpolation (as built in Matlab functions) of the spacing between the detection fibers $(0.5 \mathrm{~cm}$ spacing w.r.t each other) on the probe surface. Linear interpolation at times created multiple artifacts with no distinct target. Hence higher order polynomial fittings were attempted to enhance the representation of the 2D surface images. Using simulation studies involving phantoms of $5.5 \times 5.5 \times 10 \mathrm{~cm}^{3}$ with single targets, it was observed that 5 th degree polynomials yielded the best fit and localization of the target location (Appendix A,B). The procedure is performed by initially extracting the data of the first column of probe data and fitting the data with the 5th degree polynomial, and continue the same process for all the remaining columns of the probe data. This entire process is repeated row-wise for the entire probe data, using the 
column-wise fitted data. The procedure is then repeated for the row data for all the newly generated 1-n rows. Hence the polynomial fitting is a double regression algorithm implementing a 5th degree polynomial applied to the experimental data (after subtraction and removal of detectors nearest the sources). Implementation of the polynomial fitting in order to generate 2D contour surface images reduces the number of artifacts. Polynomial fitting reduces the number of artifacts by mitigating the spurious effects by single detectors (i.e. reduces the intensity value of single detectors surrounded by detectors with intensity values much lower).

\subsubsection{Target location and size}

To determine target location and size, the point of max intensity or centroid on the $2 \mathrm{D}$ surface contour plot is located based upon the polynomial fitting (figure $30 \mathrm{~A}$ ). The size of the target is estimated by the Full-width half max (FWHM) values which are calculated for both $\mathrm{x}$ - and $\mathrm{y}$ axis of the polynomial fitted experimental data and averaged (diameter $\mathrm{cm}$ ) (Figure $30 \mathrm{~B}, \mathrm{C}, \mathrm{D}$ ). The original target size of a $0.45 \mathrm{cc}$ sphere is $\sim 0.95 \mathrm{~cm}$ in diameter. With the diffusion of light signal it is expected that the calculated full width at half max is larger than this value at shallow depths and increase as target depth increases. A 0.45 cc spherical target was employed in experimental cases (with targets) as the typical tumor size which conventional X-ray mammography can detect consistently, $(1 \mathrm{~cm}$ diameter) where smaller lesions are detected through palpation [40]. 


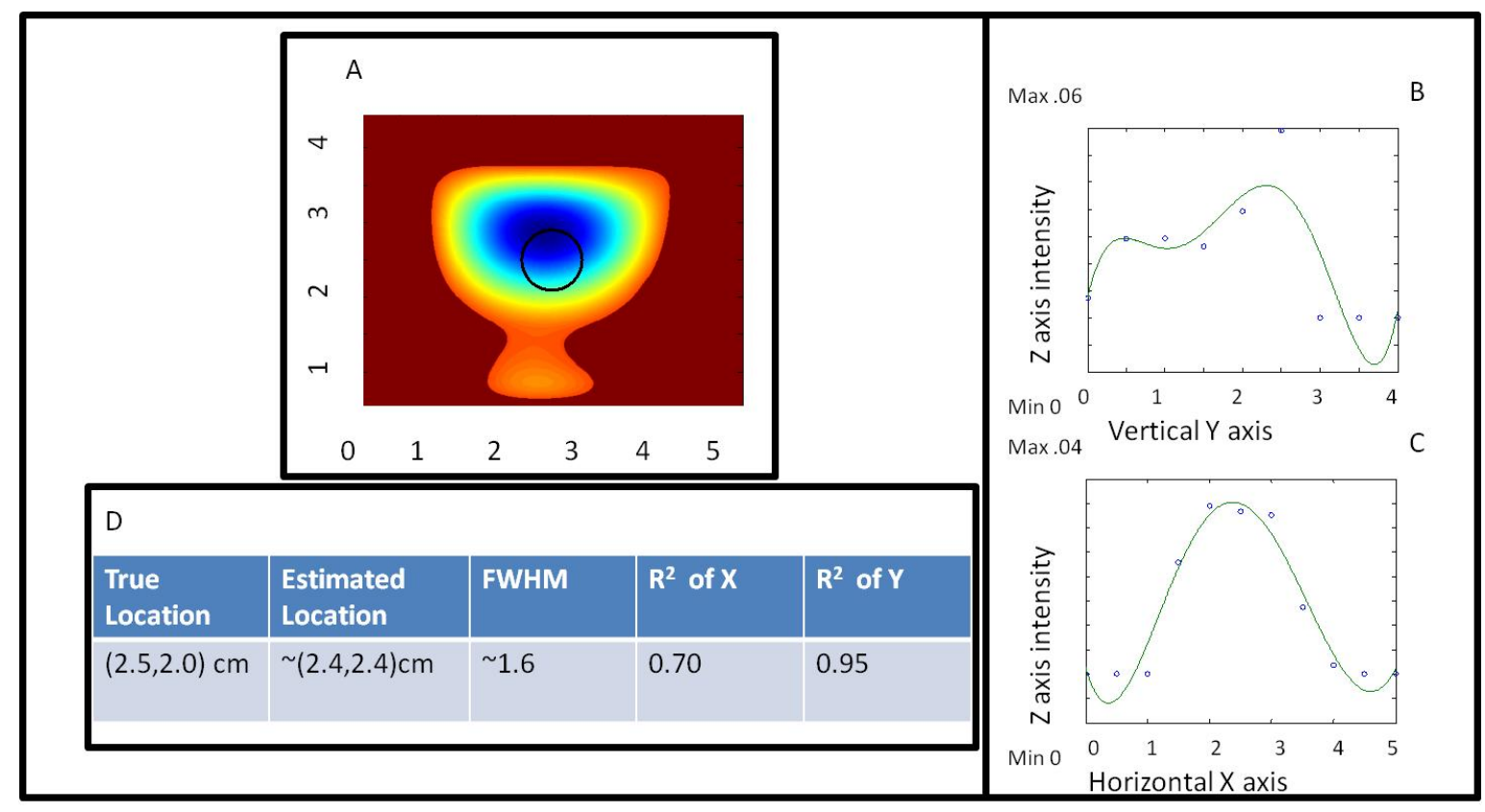

Figure 30: (A) 2D surface contour plot of a $0.45 \mathrm{cc}$ target embedded into a liposyn solution after processing. (B,C) The column and row of the 2D surface contour plot based on the centroid or the point of maximum intensity. (D) The data generated from the column and row data in $(\mathrm{cm})$ is the estimated location, Full width at half maximum or estimated size and the $r$ squared when comparing the original row and column data to the 2D-surface contour plot.

When true target location is known (as in all tissue phantom cases, and less so in breast phantom and in-vivo breast tissues as will be described in later chapters), the estimated target location is approximated by using the $\mathrm{x}, \mathrm{y}$ location of the maximum point of the polynomial plot. Additionally a target is labeled as localized when the estimated target location is within a distance of $1 \mathrm{~cm}$ of the true target location. A distance of $1 \mathrm{~cm}$ is employed as a cut-off value for target detection. The Gen-2 imaging probe has detectors spacing of $0.5 \mathrm{~cm}$ where the target employed was $0.95 \mathrm{~cm}$ in diameter. In order to compensate for possible human error during the positioning of the target a cut-off value less than 2 detector spacing ( diameter of the target or 1 $\mathrm{cm}$ ) is employed. The cut-off is employed systematically for all relevant experimental cases where the true target location is known and undisturbed during the study. 
When true target location is not available as is the case with solid phantoms and human subject studies, the criteria for localization is to see an area of absorption moving in the opposite direction of scanning. The absorption following such an event was thought to be only from the target, as artifacts would tend to either be fixed in a certain region w.r.t hand-held probe or random.

Localization of the target or region of high absorbance will eventually have a direct effect in patient diagnosis. A region of absorbance detected $1 \mathrm{~cm}$ off from the true location could potentially influence the decision of the medical professional employing the device and technology. Future work for this device will include implementation of a multi-scanning procedure and development of $3 \mathrm{D}$ tomographic reconstruction in conjunction with spatial tracking of the probes. Implementation of those features will reduce the likelihood of incorrect localization of the region of high absorption enhancing the efficiency of the device in diagnosis and prognosis. 


\subsubsection{Measurement error}

The measurement errors for each experimental case were evaluated from the average standard deviation across the given number of repeated measurements (typically 5 or 10) across all the detector points (figure 31).

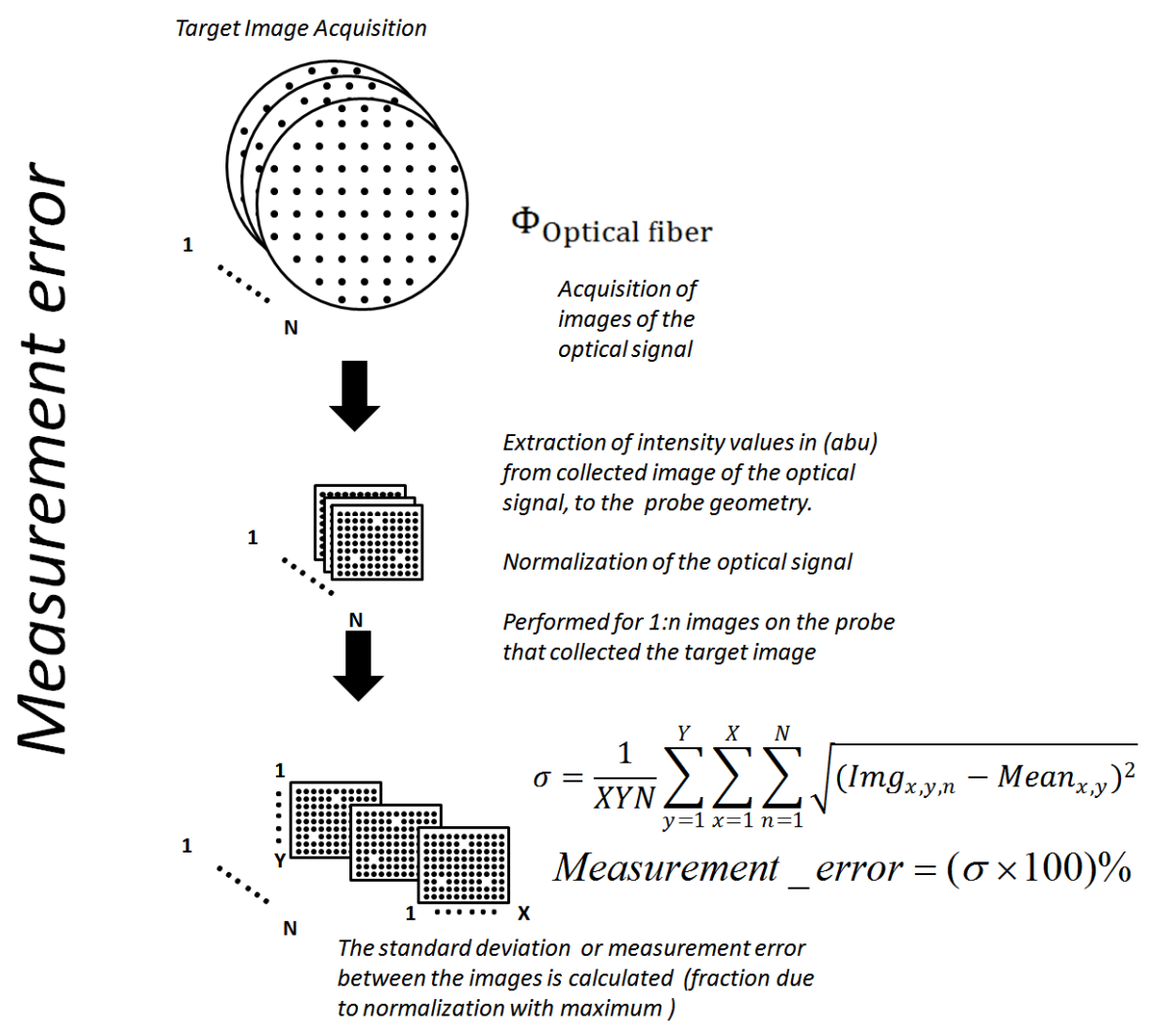

Figure 31:Flowchart for the calculation of measurement error

Measurement error is calculated by collecting the target image ( $\mathrm{n}$ - repetitions), then extracting the data to the proper detector location on the probe face and normalizing the data with the highest intensity (normalization makes the data a fraction). The standard deviation for each detector is calculated through the $\mathrm{n}$ repetitions (number of images acquired), and summed. The summed is then averaged dividing by the total number of detectors. The fraction is converted to a percent and employed as a metric of system stability. 


\subsubsection{Contrast calculation}

Contrast is calculated using processed data (subtracted data) from step 5 in figure 28 i.e. prior to polyfitting. The subtracted optical signal from detectors within $0.70 \mathrm{~cm}(1 \mathrm{~cm}$ in total distance from a center point since target diameter is $0.95 \mathrm{~cm}$ ) of the true target location are averaged and divided by the average of all the other detectors not included within $0.70 \mathrm{~cm}$. The contrast provides a quantification of the difference between target and background.

\subsection{Feasibility Studies:}

Experimental studies were performed to assess the feasibility of the Gen-2 imager towards optical signal collection and image generation. The studies conducted included dual probe imaging of a fluorescence target, single probe imaging of an absorption target, and dual probe bilateral imaging of an absorptive target.

\subsubsection{Feasibility of dual probe imaging using a fluorescence target}

Single phantom imaging (both probe on one phantom) (Figure 32) simplifies acquiring the calibration points, ensures that the target is located within the imaging surface and that an equivalent amount of light is collected by the optical detection fibers of each probe. Hence, single phantom imaging is a simple probe/phantom orientation which increases the return light collection and target detectibility. Single phantom imaging was the imaging setup chosen as to facilitate target detection to determine the feasibility of imaging a fluorescence target with the Gen-2 hand-held optical imaging system.

The two probes were placed on a $10 \times 10 \times 10 \mathrm{~cm}^{3}$ phantom side by side. A target was inserted into the $1 \%$ liposyn phantom at around $(8.5,2,2) \mathrm{cm}$ and later at around $(2.5,2,2,2) \mathrm{cm}$. 


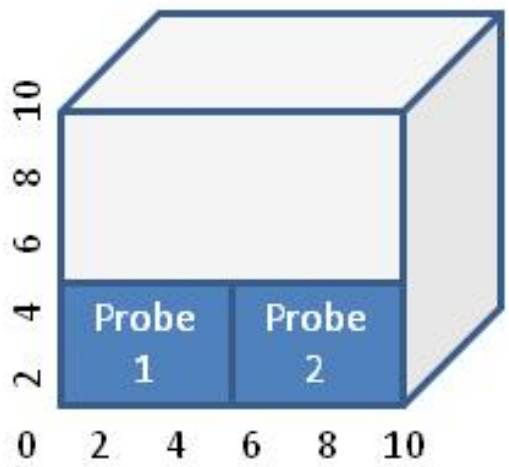

Figure 32: Dual probe imaging setup on a single phantom

The target $(0.45 \mathrm{cc})$ was filled with Indocyanine green (ICG) a FDA approved fluorescence contrast agent. A concentration of $0.1 \mu \mathrm{M}$ was employed. ICG has an excitation peak around $785 \mathrm{~nm}$ and emission peak around $830 \mathrm{~nm}$ hence fluorescence filters were employed to collect the emission. The camera collected 5 images per acquisition at a $0.2 \mathrm{msec}$ exposure time. The laser sources were optimized to output an optical power of $2 \mathrm{~mW}$ with a mean and standard deviation of around $2.05 \pm 0.060 \mathrm{~mW}$. The images were collected and processed with the subtraction imaging technique (target - background) (see section 3.2).

In Figure 33, a 2D contour plot processed via subtraction imaging (section 3.3) contains a black circle added to help locate the target $(8.5,2,2) \mathrm{cm}$ on a $10 \times 10 \times 10 \mathrm{~cm}^{3}$ cubical phantom. The experiment confirmed that second Generation hand-held optical imaging system could localize a target, in a similar case as was performed with the first Generation hand-held optical imaging system (previously). 


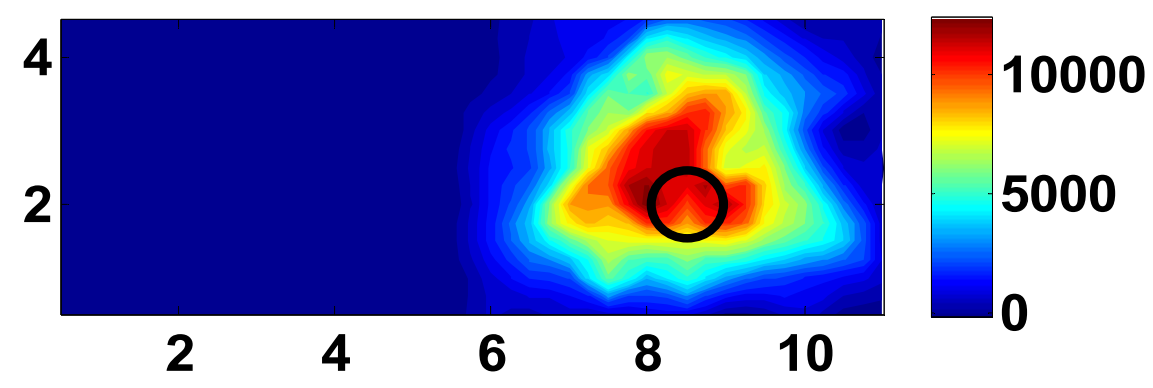

Figure 33: 2D surface contour plot of the detected fluorescence intensity of a cubical tissue phantom containing a $0.45 \mathrm{cc} 1 \mu \mathrm{M}$ ICG target (fluorescence) target at $(8.5,2,2) \mathrm{cm}$ of the liposyn phantom

After successfully locating a target filled with ICG, the focus of the experiments shifted from fluorescence based imaging to endogenous absorption contrast (as caused by angiogenesis).

\subsubsection{Feasibility of single probe imaging of an absorption target}

Single probe imaging (Figure 34) was employed to image an absorbing contrast agent, $(0.08 \%$ India Ink (Chartpak, Leeds, MA.)) which was used to simulate endogenous tissue contrast found on tumors with the second Generation hand-held optical imaging system. Single probe imaging was chosen as to later be able to directly compare the results from the first and second Generation hand-held optical imaging systems (section 4.3).

A 0.45 cc spherical target was employed with $0.08 \%$ India Ink (absorbing contrast agent) and positioned at various locations within the phantom. The camera was set to collect 10 continuous wave images per acquisition at a $0.2 \mathrm{msec}$ exposure time. A single probe was placed on the $10 \times 10 \times 10 \mathrm{~cm}^{3}$ cubical phantom, while the other probe is left covered on the side and hence not used in the imaging studies (Figure 35). Images were collected locating the target from $1 \mathrm{~cm}$ to $4 \mathrm{~cm}$ at $0.5 \mathrm{~cm}$ increments on the desired axis while leaving the others fixed. The $\mathrm{Z}$ axis position was shifted so that the target position resembles $(3,1.5, Z) \mathrm{cm}$, where $\mathrm{Z}$ is the only variable and the $\mathrm{X}, \mathrm{Y}$ position of the target is always $(3,1.5)$ respectively. 


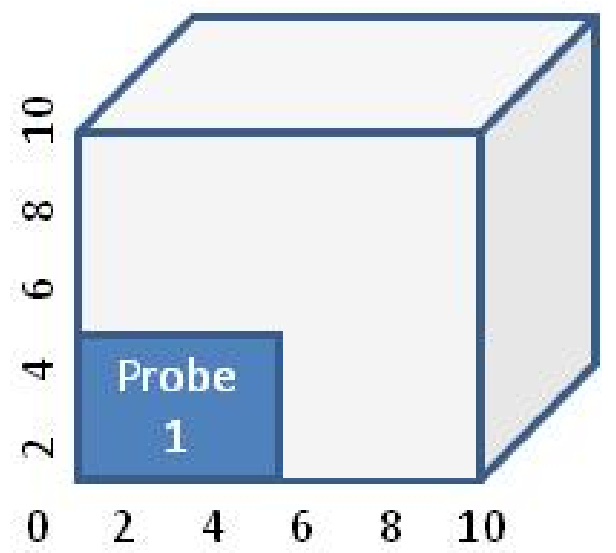

Figure 34: Single Probe imaging setup on a single phantom

The experiment determined if an India Ink target of different concentrations could be localized. The Figure 35 is a $2 \mathrm{D}$ contour plot of detected optical intensity, generated using subtraction image processing that shows two cases where depth placement was 1.5 and $2 \mathrm{~cm}$.

(A)

Target position

$(3 \cdot 0,1.5,1.5) \mathrm{cm}$

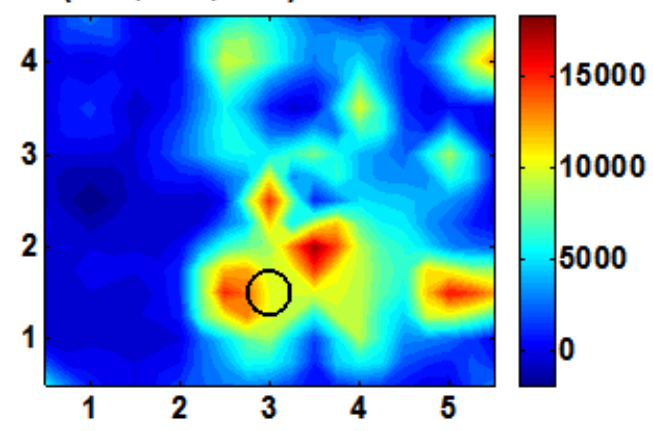

(B)

\section{Target position}
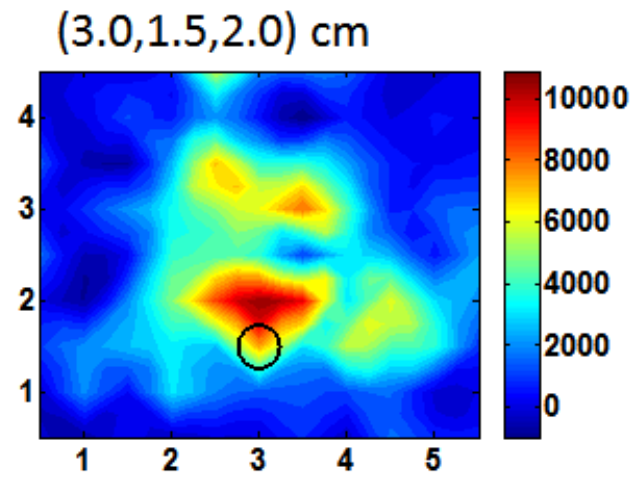

Figure 35: 2D surface contour plot of detected optical intensity of a cubical phantom containing a India Ink target ( $0.08 \%$ by volume) inside a liposyn solution, where the true trarget location is demarcated by the black circle is located at (A) $1.5 \mathrm{~cm}$ deep target, (B) $2.0 \mathrm{~cm}$ deep target. 
A $2 \mathrm{~cm}$ depth collection was similar to what had been detected by the first Generation hand-held optical imaging system [30]. The images collected are subject to artifacts that could have been due to an instrument effect such as the lens assembly sliding which results in a change of the calibration points, thus introducing the artifacts during processing the images. Other factors possibly affecting the artifacts on figure 35 are liposyn variability (liposyn settles after some time which for a single image would not be a factor but a background image is also acquired) and target variability. Target variability such as placing and replacing the target over the course of an experiment could lead to placement errors and also mixes the liposyn in the area where the target was placed introducing liposyn variability. Thus it is difficult to conclude that the maximum target detectability depth is $2 \mathrm{~cm}$, regardless which target localization is possible using absorption contrast agents.

\subsubsection{Feasibility of dual probe bilateral imaging of an absorptive target (Bilateral Imaging:}

Simultaneous vs. Sequential)

Bilateral imaging (Figure 36) fully utilizes the dual probe design on the second Generation handheld optical imaging system and is applicable to both phantom and in-vivo studies. Bilateral imaging allows for a comparison between two distinct tissue regions given that breast cancer is typically unilateral. Hence, bilateral imaging allows for comparison of the diseased breast tissue with the contralateral breast (healthy breast) [41]. Comparison with the contralateral breast allows the patient to serve as both the target (diseased breast tissue) and the background (healthy breast tissue) since both breasts should have similar optical properties for healthy breast tissue. Hence the major benefit of bilateral imaging is having each patient serve as their own comparison. In the current thesis, bilateral imaging results will only be presented for bilateral phantom imaging. A absorbing contrast agent (0.02\% India Ink Chartpak, Leeds, MA) was used 
to simulate endogenous tissue contrast found on tumors. The two methods that will be used to process the images collected are simultaneous and sequential bilateral imaging (section 3.3), which are modified processing techniques based on subtraction that do not require the target to be removed (which is not possible in-vivo).

The experimental set up involved two cubical phantoms with dimensions $10 \times 10 \times 10 \mathrm{~cm}^{3}$ filled with $1 \%$ liposyn to simulate human breast tissue (Figure 55). A $0.45 \mathrm{cc}$ spherical target containing $0.02 \%$ India Ink (absorbing contrast agent Chartpak, Leeds, MA) The exposure time of the camera was again set to 0.2 seconds and collected a total of 10 images per acquisition. Each probe is placed on a separate phantom and imaging is performed. The experimental setup simulates the two breast tissues (phantoms) with a unilateral tumor (a target placed in one of the phantoms). Section 3.2 describes the processing methods for bilateral imaging.
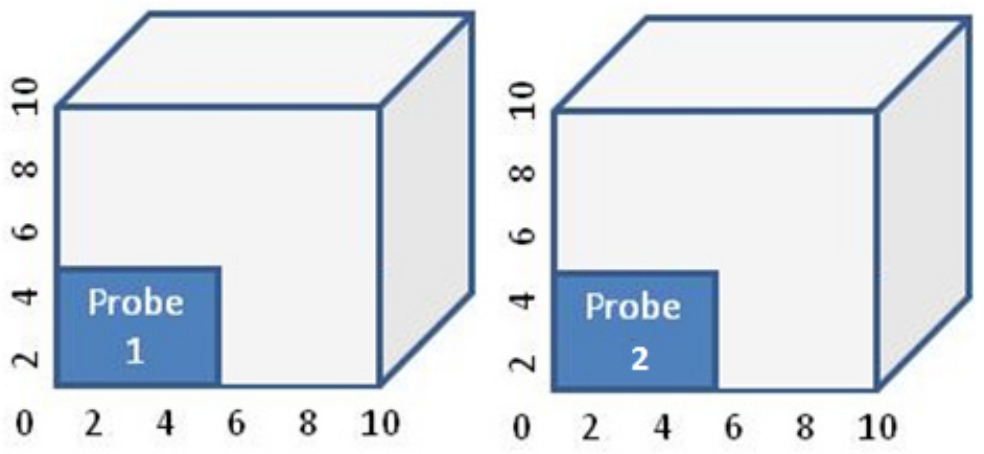

Figure 36: Bilateral imaging setup on two phantoms

Figure 37 contains the images at each step described in section 3.2 (Figure 28). Step 1 and 2 are the images collected from the tissue surface (without processing); the images are the signal intensity distribution of the probe face. The images from Step 1 and 2 are processed (as explained in section 3.4,3.5) to gather Step 5 and 6, which are the results from simultaneous and 
sequential bilateral imaging respectively. Step 4 (variability within the probes, Figure 37) displays the pattern that is subtracted from Step 3(variability of the phantoms and variability of the probes, Figure 28). The pattern which if proven to be consistent (same for all images after normalization) would allow simultaneous bilateral imaging to require only step 1 (figure 28) and not step 2 to produce a calibrated (the pattern) subtracted image (step 6). Step 7 (Figure 37) is a (side by side) comparison of simultaneous and sequential bilateral imaging yielding results similar for both images. 


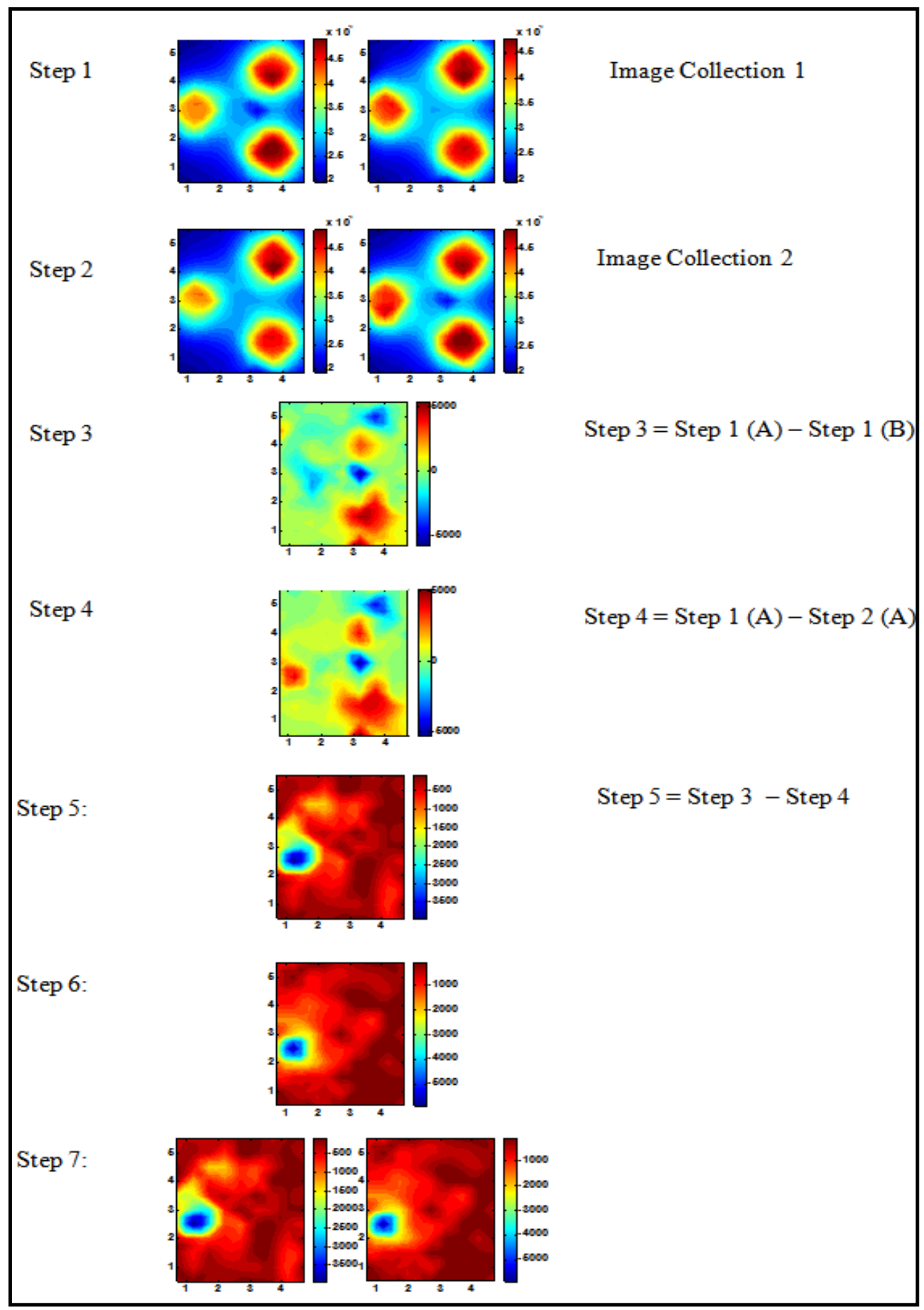

Figure 37: Bilateral Imaging of two identical tissue phantoms with embedded India Ink $0.02 \%$ target filled with $1 \%$ liposyn solution. The images are 2D contour plots of NIR detected signal which result from simultaneous and sequential processing. 
The target is localized in the same location for both bilateral imaging techniques (simultaneous and sequential) with a minimum of artifacts. It suggests that both imaging techniques are equivalent and hence both viable towards unilateral optical imaging of breast tissue, as shown inFigure 37, Step 7. Since breast tumors are generally unilateral (almost $75 \%$ of the the cases) this study suggests that either simultaneous or sequential processing would be successful in localizing tumors in-vivo.

\subsection{Summary}

Chapter 3 has described the devices and products chosen in the development of a Gen-2 imager (section 3.1). Along with the instrumentation that makes up the Gen-2 imager the processing, image acquisition and target detection criteria of the imager were also described. The entire process of image acquisition and processing is displayed on figure 24. While optical signal collection, image generation and processing were shown to be feasible using both probes on fluorescence and absorption based targets. The next chapter introduces the first series of experimental results which compare the Gen-1 and Gen-2 hand-held optical imagers. 


\section{CHAPTER 4: Gen-1 and Gen-2 comparison}

\subsection{Introduction}

The Gen-1 imager was not only bulky and non-patient comfortable, but was also challenging for in-vivo clinical applications being limited by non-homogenous illumination resulting in the creation of artifacts. Herein, a Generation-2 (Gen-2) hand-held optical imager is developed as a portable device with improved features appropriate towards (future) in-vivo breast imaging studies and clinical translation of the technology. The effect of the improved instrumentation in enhancing target detectability is experimentally validated from tissue phantom studies and the potential clinical applications of the device are highlighted.

\subsection{Materials and Methods}

\subsubsection{Instrumentation}

A Generation-2 or Gen-2 hand-held probe has been developed with features that would overcome the limitations of Gen-1 hand-held probe (described in chapter 2 and shown in Table 4). The instrumentation associated with the Gen-2 imager is found in section 3.1 where the sources, probe and detectors are discussed in detail.

\subsubsection{Experimental studies}

Experimental tissue phantom studies were performed in order to demonstrate the effectiveness of the Gen-2 hand-held optical imager over the Gen-1 imager in terms of its target detectability (that may be impacted from the uniformity or non-uniformity of source strength distribution). A cubical phantom of $10 \times 10 \times 10 \mathrm{~cm}^{3}$ was filled with $1 \%$ Liposyn solution (Hospira, Lake Forest, IL) with optical properties mimicking that of a breast tissue. A $0.45 \mathrm{~cm}^{3}(\sim 0.95 \mathrm{~cm}$ diameter $)$ tumor-simulating target filled with $.08 \%$ (by volume) India Ink (an absorption contrast agent) in 
$1 \%$ Liposyn solution was placed at various locations and a depth of $1.0 \mathrm{~cm}$ from the imaging surface. The absorption $\left(\mu_{\mathrm{a}}\right)$ and scattering coefficient $\left(\mu_{\mathrm{s}}^{\prime}\right)$ of $1 \%$ Liposyn as measured using a homodyne single-pixel based frequency domain system was $0.1 \pm 0.17 \mathrm{~cm}-1$ and $\sim 2.52 \pm 0.23 \mathrm{~cm}$ 1 , respectively $\left(\right.$ where $\mu_{\mathrm{s}}{ }^{\prime}>>\mu_{\mathrm{a}}$ ). The absorption coefficient of $0.08 \%$ India Ink was estimated using a spectrophotometer (originally attempted in a homodyne device Appendix E) as $\sim 0.07$ $\mathrm{cm}-1$ at $785 \mathrm{~nm}$ wavelength). The scattering coefficient $\left(\mu_{\mathrm{s}}^{\prime}\right)$ of India Ink can vary between 4 to $20 \mathrm{~cm}-1$ as reported by various researchers [42-44], and was challenging to estimate in our studies. The optical properties of India ink were difficult to quantify due containing a high amount of absorption and non-negligible amount of scattering at the concentrations employed $(0.8,0.08$, and 0.008 by volume $)$ distorting the measurements performed by a homodyne device. Other research groups such as Madsen et all reported similar effects while trying to determine the optical properties of India ink which could lead to overestimation of absorption and underestimation of scattering [44]. The typical optical properties of human breast tissues are $\mu_{\mathrm{a}}=$ $0.04 \pm 0.02 \mathrm{~cm}-1, \mu_{\mathrm{s}}^{\prime}=8 \pm 4 \mathrm{~cm}-1$ [45-48]. Hence, the phantoms used in the current studies have similar optical properties of a human breast tissue. Simultaneous multiple point illumination was carried out using both Gen-1 and Gen-2 imagers. Laser diodes at $785 \mathrm{~nm}$ wavelength were used at an output optical power of $2 \mathrm{~mW}$ in each source. Figure 18 displays the intensity distribution that was observed for the Gen-1 and Gen-2 imagers where the mean and

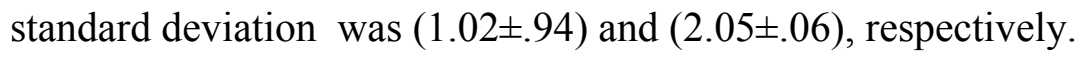

Continuous wave measurements of attenuated near-infrared signals were acquired by the ICCDbased detector in both the imagers. In Gen-1 and Gen-2 imager, the exposure time was set to 0.2 sec and 5 repeated measurements were acquired (and averaged) at each probe location on the tissue phantom surface. Since the focus of this study was only to evaluate the effect of uniform 
source distribution on target detectability, only one probe head of the two heads was used during imaging studies in the Gen-2 imager. Prior to performing the imaging studies, the source strengths of the simultaneous sources were measured using an optical power meter (Thorlabs Inc., Newton, NJ).

The CW-based raw intensity signals acquired by the detectors in both the imagers was postprocessed in order to determine the $2 \mathrm{D}$ intensity distribution map of the imaged surface (as $2 \mathrm{D}$ surface contour plots). Experimental studies were performed with the target placed at various locations, w.r.t to the source locations. For consistency, the target's location was shifted horizontally in the x-axis, keeping its vertical position (y-axis) and depth (z-axis) constant, and optical signals were acquired at each of the target's location.

\subsubsection{Data analysis}

Subtraction and removal of detectors around the sources was applied to the optical signals as explained in chapter 3 sections. A measure of FWHM was included as measured from the detected target's centroid. Measurement errors for each of the experimental cases are shown as the average standard deviation of the 5 repeated measurements obtained across all the detectors of the Gen-1 and Gen-2 probes.

\subsection{Results and Discussion}

The improvement of the Gen-1 hand-held optical imaging system in terms of the hand-held design, source illumination system, and the compactness of the system (from a bench-top setup) has led to the evolution of a more clinically applicable and portable Gen-2 hand-held optical imager. The effectiveness of the Gen-2 over Gen-1 imager towards improved 2D target detectability is experimentally evaluated. The output powers of the simultaneous illuminating sources in both generations of the hand-held imager are shown in Figure 5. The source 
intensities in Gen-1 probe vary significantly across the 6 sources with a $94 \%$ standard deviation and an average power of $1.02 \mathrm{~mW}$. In comparison, the Gen-2 probes have significantly consistent source intensities across the 3 sources in each probe head, with a $3 \%$ standard deviation and an average power of $2.01 \mathrm{~mW}$.

Figure 38 shows the 2D surface contour plots of the detected signals using the Gen-1 imager for two experimental cases, where the target was placed $\mathrm{z}=1.0 \mathrm{~cm}$ deep, but at different $\mathrm{x}-\mathrm{y}$ locations in the cubical phantom.
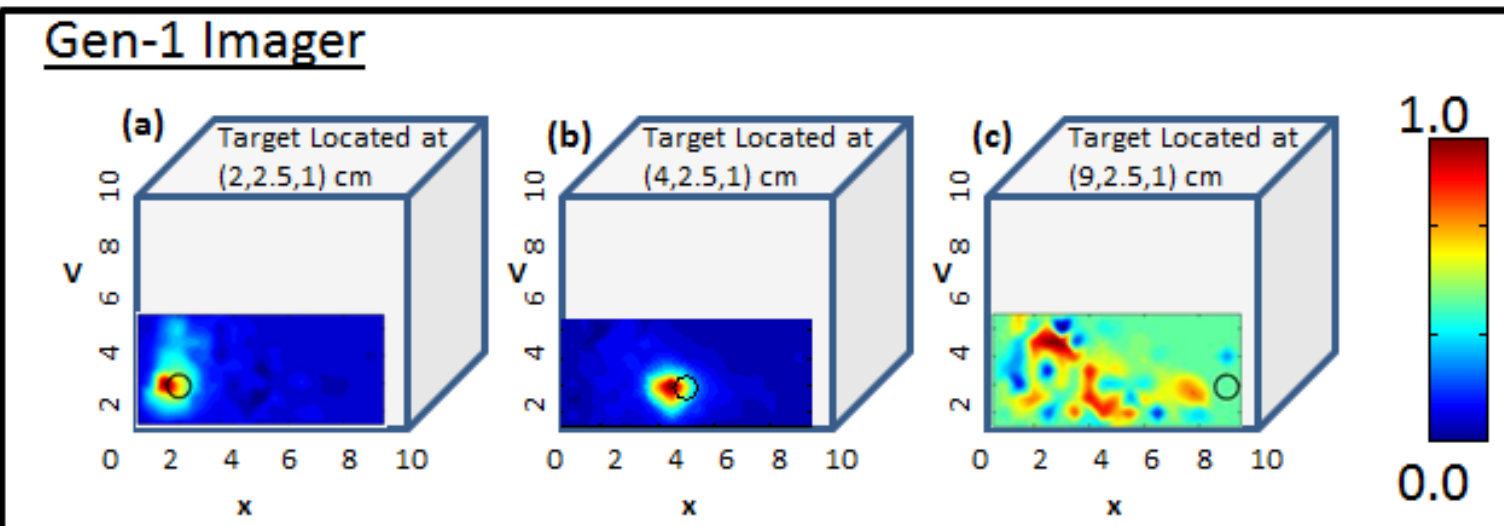

Figure 38: The Gen-1 imager produced 2D surface plots after processing of the optical signal collected during an experimental study involving a $0.45 \mathrm{cc}$ India ink target placed in a cubical $10 \times 10 \times 10 \mathrm{~cm}^{3}$ phantom filled with $1 \%$ liposyn. The target was placed at a) $(2,2.5,1) \mathrm{cm} \mathrm{b})(4,2.5,1) \mathrm{cm}$ and c) $(9,2.5,1) \mathrm{cm}$.

Although experiments were performed at various target $\mathrm{x}$-locations along the same $\mathrm{y}-\mathrm{z}$ plane, only three experimental cases are shown here for brevity. When the target was located at $(2,2.5,1)$ or $(4,2.5,1) \mathrm{cm}$, it was closer to two relatively strong sources $(2.169 \mathrm{~mW}, 1.03 \mathrm{~mW}$, or $2.087 \mathrm{~mW}$ ). The detected (and subtracted) optical signals were able to detect the target distinctly and closer to its true location (see Figures 38a, 38b and Cases 1 and 2 of Table 7). 


\begin{tabular}{|c|c|c|c|c|c|}
\hline Imaging System & $\begin{array}{l}\text { True Target Location } \\
(\mathrm{x}, \mathrm{y})(\mathrm{cm})\end{array}$ & $\begin{array}{l}\text { Detected } \\
\text { location }(\mathrm{x}, \mathrm{y}) \\
(\mathrm{cm})\end{array}$ & $\begin{array}{l}\text { Distance off } \\
\text { from true } \\
(\mathrm{x}, \mathrm{y}) \text { location } \\
(\mathrm{cm})\end{array}$ & $\begin{array}{l}\text { Estimated } \\
\text { target } \\
\text { diameter }(\mathrm{cm})\end{array}$ & $\begin{array}{l}\text { Measurement } \\
\text { error }\end{array}$ \\
\hline \multicolumn{6}{|l|}{ Gen-1 $(\operatorname{Exp} \#)$} \\
\hline 1 & $(1.0,2.0)$ & $(1.0,1.5)$ & 0.5 & 1.2 & 0.0022 \\
\hline 2 & $(4.0,2.0)$ & $(4.0,1.5)$ & 0.5 & 1.3 & 0.0019 \\
\hline 3 & $(7.0,2.0)$ & $(1.5,2.5)$ & 5.7 & 1.7 & 0.0024 \\
\hline 4 & $(8.0,2.0)$ & $(5.0,3.5)$ & 3.6 & 0.34 & 0.0026 \\
\hline 5 & $(9.0,2.0)$ & $(1.5,3.5)$ & 6.6 & 1 & 0.0028 \\
\hline \multicolumn{6}{|l|}{ Gen-2 (Exp \#) } \\
\hline 1 & $(1.0,2.5)$ & $(0.5,2.0)$ & 0.5 & 1.8 & 0.0098 \\
\hline 2 & $(2.0,2.5)$ & $(1.5,2.0)$ & 0.7 & 1.3 & 0.014 \\
\hline 3 & $(2.5,2.5)$ & $(2.0,2.5)$ & 0.5 & 0.76 & 0.0060 \\
\hline 4 & $(3.0,2.5)$ & $(2.5,2.5)$ & 0.5 & 0.99 & 0.015 \\
\hline 5 & $(4.0,2.5)$ & $(3.5,2.5)$ & 0.5 & 1.1 & 0.021 \\
\hline
\end{tabular}

Table 7: Quantitative details of the detected target location, size and measurement errors for different experimental cases using the Gen-1 and Gen-2 imager.

On the contrary, when the target was located at $(9,2.5,1) \mathrm{cm}$, it was closer to two relatively weaker sources $(0.053 \mathrm{~mW}$ and $0.715 \mathrm{~mW})$. The detected (and subtracted) optical signals could not detect the target (as seen from Figure 38c and Case 38 of Table 7). This is possibly because the source signals attenuate exponentially and weaker sources may eventually lead to insensitive NIR signals from the targets. Hence, in spite of subtracting the background excitation leakage signals, the optical signals from the absorption contrasted target are too weak to be detected by the ICCD camera.

Figure 39 shows the 2D surface contour plots of the detected signals using Gen-2 imager (only one probe head used) for two experimental cases, where the target was placed $\mathrm{z}=1.0 \mathrm{~cm}$ deep, but at different $x-y$ locations in the cubical phantom. 


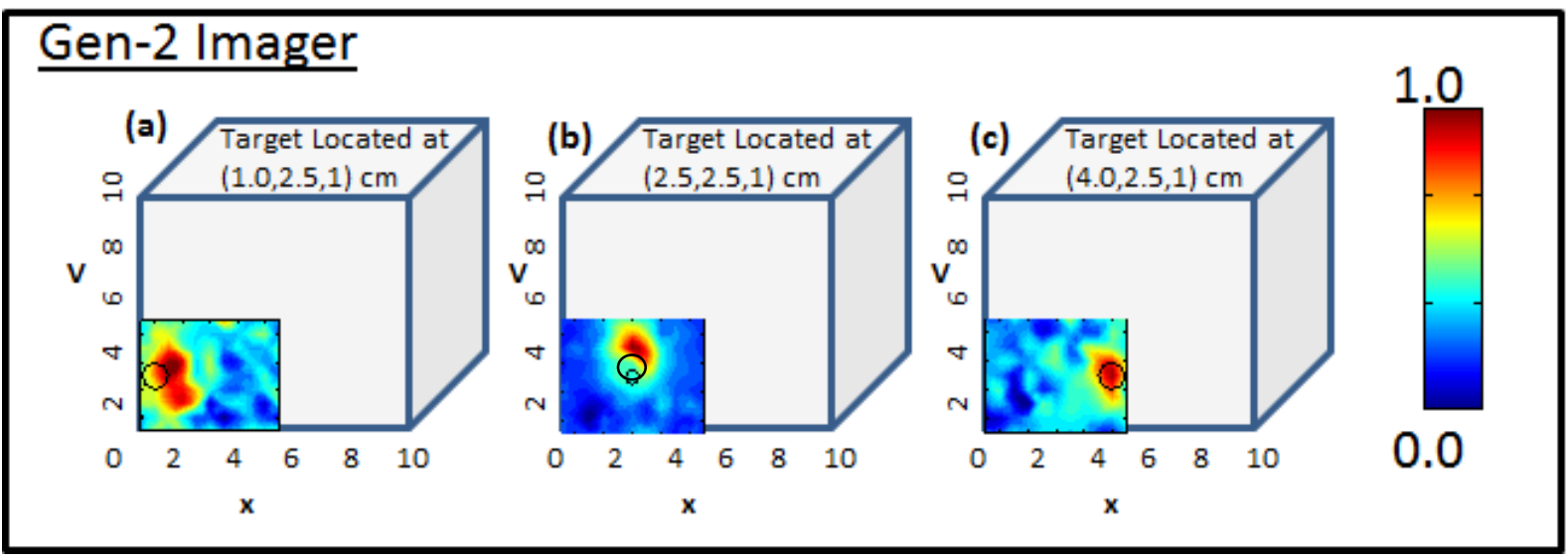

Figure 39: The Gen-2 imager produced the 2D surface plots after processing of the optical signal from an experimental study involving a $0.45 \mathrm{cc}$ India ink target placed in a cubical $10 \times 10 \times 10 \mathrm{~cm}^{3}$ phantom filled with $1 \%$ liposyn. The target was placed at a) $(1,2.5,1)$ b) $(2.5,2.5,1)$, and c) $(4,2.5,1)$.

In this study, the detected (and subtracted) optical signals were able to distinctly detect the target in 2D, at any target location w.r.t the source layout (as quantitatively shown from Cases 4-6 of Table 7). This is possibly because of the presence of strong and uniform-strength source illumination in the Gen-2 imager unlike in Gen-1, that Generate signals sensitive enough to be detected by the ICCD camera.

From Table 7, it is observed that the estimated target size (i.e. average FWHM measurements) was consistently larger than the true target size $(0.95 \mathrm{~cm}$ diameter $)$ in most experimental cases and independent of the imager used (Gen-1 or Gen-2). This is probably from the diffused optical signal, whose detected size from 2D images is impacted by the depth of the target as well. The the Gen-1 imager was an order of magnitude smaller with regards to measurement error than the Gen-2 imager at any given target location with respect to the source. This is because the measurement error is dependent on the stability of the input source light, and sensitivity of the detector (here ICCD camera) during repeated measurements. From vast experimental studies and experience, it was observed that the sensitivity of the ICCD camera played a significant role 
in impacting the measurement errors. The Gen-1's ICCD camera was a highly sensitive Gen-III image intensifier (FS9910, ITT Night Vision) optically coupled to a 16-bit CCD camera (PISCX, Princeton Instruments) and the Gen-2's ICCD camera was a customized PI-MAX II (Princeton Instruments). From the specifications of the camera and their performance, the Gen1's ICCD camera was highly sensitive and consistently demonstrated lesser measurement errors over Gen-2's camera, independent of the probe it was coupled to (i.e. Gen-1 or Gen-2 probe). In other words, while the ICCD camera affects the overall measurement errors during experimental studies, the effectiveness in improved target detectability is predominantly due to the nature of the source strength distribution from the probes.

Thus the above studies have demonstrated the effectiveness of uniform source strength distribution on target detectability during near real-time surface imaging using the Gen-2 imager. Additionally, the smooth curvable Gen-2 probe head has improved surface area contact w.r.t to a curved phantom (e.g. cylindrical) when compared to the 3-piece Gen-1 probe head. The images of Gen-1 on curved phantoms yielded excessive excitation leakage due to poor surface contact, causing direct leakage of excitation light from the sources to the detector fibers at the edges of the 3-pieace probe head.

\subsection{Conclusions}

The above studies have demonstrated the effectiveness of uniform source strength distribution on target detectability during near real-time surface imaging using the Gen-2 imager. The next chapter will discuss how reflectance and transillumination will be applied to gain deeper target detection. 
CHAPTER 5: Bilateral and Transillumination imaging on cubical phantoms

\subsection{Introduction}

The focus of the chapter is to demonstrate the feasibility of performing both reflectance and transillumination imaging using this Gen-2 hand-held optical imager. The improvement in deep target detection using transillumination measurements over reflectance is assessed. In addition, new data post-processing techniques results will be displayed and the improvement to target detectability and from surface optical measurements (both reflectance and transillumination) will be discussed.

\subsection{Materials and Methods}

\subsubsection{Instrumentation}

A Gen-2 hand-held probe has been developed with features that would overcome the limitations of Gen-1 hand-held probe (described in chapter 3 and shown in Table 4) including the ability to perform reflectance and transillumination measurements (see section 3.2:-3.3).

\subsubsection{Experimental Studies}

Diffuse optical imaging studies were performed on cubical and slab phantoms filled with 3\% Liposyn solution (Hospira, Inc. Lake Forest, IL). Spherical targets of $0.45 \mathrm{cc}$ (0.95 cm diameter) filled with $0.08 \%$ (by volume) India Ink (absorbing agent) (Chartpak, Leeds, MA.) and 3\% Liposyn solution were employed. Reflectance (simultaneous bilateral) and transillumination experiments were performed under different experimental conditions of target depth $(1-5 \mathrm{~cm})$, and India Ink target: background (T:B) contrast ratio 1:0 1000:1 100:1 and 10:1, with a India ink target of $0.8,0.08$ or $0.008 \%$ by volume (table 9,10 ) (reflectance imaging was performed up to $2.5 \mathrm{~cm}$ Appendix A-C). 
Background contrast ratios of 1:0,1000:1, 100:1 and 10:1 by volume were employed to compare reflectance and transillumination studies at different T:B ratios. In table 8 it is shown that contrast ratios do not represent true 1:0,1000:1, 100:1 and 10:1 cases since absorption contrast is employed. The background liposyn contains some absorption lowering the contrast ratios to the calculated T:B (equation 20).

$$
\text { Calculated } T: B=\frac{\mu_{\text {a Liposyn }}+\mu_{\text {a Liposyn+India ink }}}{\mu_{\text {a Liposyn }}}
$$

\begin{tabular}{|c|c|c|c|c|}
\hline & Absorption Con & t Ratios & & \\
\hline $\begin{array}{l}\text { Target }_{\text {India }} \text { Ink (vol } \\
\%)\end{array}$ & $T: B_{\text {India Ink }}=1: 0$ & 1000:1 & 100:1 & $10: 1$ \\
\hline $0.8 \%$ & 65.6 & 56.1 & 37.4 & 8.68 \\
\hline $0.08 \%$ & 7.58 & 6.79 & 6.45 & 4.31 \\
\hline $0.008 \%$ & 1.75 & 1.58 & 1.57 & 1.49 \\
\hline
\end{tabular}

Table 8: Calculated T:B ratios (factoring in the absorption coefficient of liposyn)

The combination of the targets and background contrast ratios shown as the calculated T:B range from 65:1 to 1.5:1. Every contrast ratio could not be performed. However, the chosen T:B ratios cover a wide range of possible contrast ratios. Chernomordik et al list the optical properties for 2 breast cancer cases of invasive ductile carcinoma (at $785 \mathrm{~nm}$ ), where the calculated T:B was found to be 2.95:1 $(2.6 \mathrm{~cm})$ and 2.75:1 $(2.3 \mathrm{~cm})$ after application of the equation 20 [45]. These absorption contrast ratios in actual breast tumors are within the range of absorption contrast ratios tested during our phantom studies (as shown in Table 8). 


\begin{tabular}{|c|c|c|c|c|}
\hline \multicolumn{5}{|c|}{ Reflectance: } \\
\hline \multirow{2}{*}{$\begin{array}{l}\text { Depth } \\
\text { z (cm) }\end{array}$} & \multicolumn{4}{|c|}{ Contrast ratios } \\
\hline & $1: 0$ or $(0.8 \%: 0)$ & $\begin{array}{l}\text { 1000:1 or } \\
(0.8 \%):(0.0008 \%)\end{array}$ & $\begin{array}{l}\text { 100:1 or } \\
(0.8 \%):(0.0008 \%)\end{array}$ & $\begin{array}{l}10: 1 \text { or } \\
(0.8 \%):(0.0008 \%)\end{array}$ \\
\hline 1 & Expla & Explb & Exp1c & Exp1d \\
\hline 1.5 & Exp2a & Exp2b & Exp2c & Exp2d \\
\hline 2 & Exp3a & Exp3b & Exp3c & Exp3d \\
\hline 2.5 & Exp4a & Exp4b & Exp4c & Exp4d \\
\hline $\begin{array}{l}\text { Depth } \\
\text { z (cm) }\end{array}$ & $1: 0$ or $(0.08 \%: 0)$ & $\begin{array}{l}\text { 1000:1 or } \\
(0.08 \%):(0.00008 \%)\end{array}$ & $\begin{array}{l}\text { 100:1 or } \\
(0.08 \%):(0.00008 \%)\end{array}$ & $\begin{array}{l}10: 1 \text { or } \\
(0.08 \%):(0.00008 \%)\end{array}$ \\
\hline 1 & Exp5e & Exp5f & Exp5g & Exp5h \\
\hline 1.5 & Exp6e & Exp6f & Exp6g & Exp6h \\
\hline 2 & Exp7e & Exp7f & Exp7g & Exp7h \\
\hline 2.5 & Exp8e & Exp8f & Exp8g & Exp8h \\
\hline $\begin{array}{l}\text { Depth } \\
\text { z (cm) }\end{array}$ & $1: 0$ or $(0.008 \%: 0)$ & $\begin{array}{l}\text { 1000:1 or } \\
(0.008 \%): \\
(0.000008 \%)\end{array}$ & $\begin{array}{l}100: 1 \text { or } \\
(0.008 \%): \\
(0.000008 \%)\end{array}$ & $\begin{array}{l}10: 1 \text { or } \\
(0.008 \%): \\
(0.000008 \%)\end{array}$ \\
\hline 1 & Exp9i & Exp9j & Exp9k & Exp91 \\
\hline 1.5 & Exp10i & Exp10j & Exp10k & Exp101 \\
\hline 2 & Exp11i & Exp11j & Exp11k & Exp111 \\
\hline 2.5 & Exp12i & Exp12j & Exp12k & Exp121 \\
\hline
\end{tabular}

Table 9: Experimental studies using reflectance imaging using a $.45 \mathrm{cc}$ India ink target (concentration of India ink included $0.8,0.08$ and $.008 \%$ by volume) on a $10 \times 10 \times 10 \mathrm{~cm}^{3}$ liposyn phantom $(1: 0,1000: 1,100: 1$, and 10:1 target to background contrast ratios). Cases where successful target localization (Within $1 \mathrm{~cm}$ of true target location) occurs are displayed by shaded gray. 


\begin{tabular}{|c|c|c|c|c|}
\hline \multicolumn{5}{|c|}{ Transillumination } \\
\hline \multirow[b]{2}{*}{$\begin{array}{l}\text { Depth z } \\
\text { (cm) }\end{array}$} & \multicolumn{4}{|c|}{ Contrast Ratios } \\
\hline & $1: 0$ or $(0.8 \%: 0)$ & $\begin{array}{l}\text { 1000:1 or } \\
(0.8 \%):(0.0008 \%)\end{array}$ & $\begin{array}{l}100: 1 \text { or } \\
(0.8 \%):(0.0008 \%)\end{array}$ & $\begin{array}{l}10: 1 \text { or } \\
(0.8 \%):(0.0008 \%)\end{array}$ \\
\hline 1 & Exp1a & Exp1b & Exp1c & Exp1d \\
\hline 2 & Exp2a & Exp2b & Exp2c & Exp2d \\
\hline 3 & Exp3a & Exp3b & Exp3c & Exp3d \\
\hline 4 & Exp4a & Exp4b & Exp4c & Exp4d \\
\hline 5 & Exp5a & Exp5b & Exp5c & Exp5d \\
\hline $\begin{array}{l}\text { Depth z } \\
\text { (cm) }\end{array}$ & 1:0 or $(0.08 \%: 0)$ & $\begin{array}{l}\text { 1000:1 or } \\
(0.08 \%):(0.00008 \%)\end{array}$ & $\begin{array}{l}100: 1 \text { or } \\
(0.08 \%):(0.00008 \%)\end{array}$ & $\begin{array}{l}10: 1 \text { or } \\
(0.08 \%):(0.00008 \%)\end{array}$ \\
\hline 1 & Exp6e & Exp6f & Exp6g & Exp6h \\
\hline 2 & Exp7e & Exp7f & Exp7g & Exp7h \\
\hline 3 & Exp8e & Exp8f & Exp8g & Exp8h \\
\hline 4 & Exp9e & Exp9f & Exp9g & Exp9h \\
\hline 5 & Exp10e & Exp10f & Exp10g & Exp10h \\
\hline $\begin{array}{l}\text { Depth z } \\
\text { (cm) }\end{array}$ & $\begin{array}{l}\text { 1:0 or } \\
(0.008 \%: 0)\end{array}$ & $\begin{array}{l}\text { 1000:1 or } \\
(0.008 \%): \\
(0.000008 \%)\end{array}$ & $\begin{array}{l}100: 1 \text { or } \\
(0.008 \%): \\
(0.000008 \%)\end{array}$ & $\begin{array}{l}10: 1 \text { or } \\
(0.008 \%): \\
(0.000008 \%)\end{array}$ \\
\hline 1 & Exp11i & Exp11j & Exp11k & Exp111 \\
\hline 2 & Exp12i & Exp12j & Exp12k & Exp121 \\
\hline 3 & Exp13i & Exp13j & Exp13k & Exp131 \\
\hline 4 & Exp14i & Exp14j & Exp14k & Exp141 \\
\hline 5 & Exp15i & Exp15j & Exp15k & Exp151 \\
\hline
\end{tabular}

Table 10: Experimental studies using transillumination imaging using a .45 cc India ink target (concentration of India ink included $0.8,0.08$ and $.008 \%$ by volume) on a $10 \times 10 \times 10 \mathrm{~cm}^{3}$ liposyn phantom $(1: 0,1000: 1,100: 1$, and 10:1 target to background contrast ratios). Cases where successful target localization (Within $1 \mathrm{~cm}$ of true target location) occurs are displayed by shaded gray. 
During simultaneous bilateral reflectance imaging studies, two $10 \times 10 \times 10 \mathrm{~cm}^{3}$ cubical phantoms were used, one with a target and the other without. During transillumination imaging studies, $5.5 \times 5.5 \times 10 \mathrm{~cm}^{3}$ slab phantoms were used. All the 3 point sources of one probe head are simultaneously illuminated $(\mathrm{ON})$ and the sources on the other probe head (located on the opposite surface of the imaging plane) are not (other probe OFF) (see Figure $40 \mathrm{~B}$ ). These studies were to determine if transilluminated measurements detect deeper targets (from 2D surface images) over reflected measurements upon using a unique hand-held probe capable of acquiring both these measurement types.

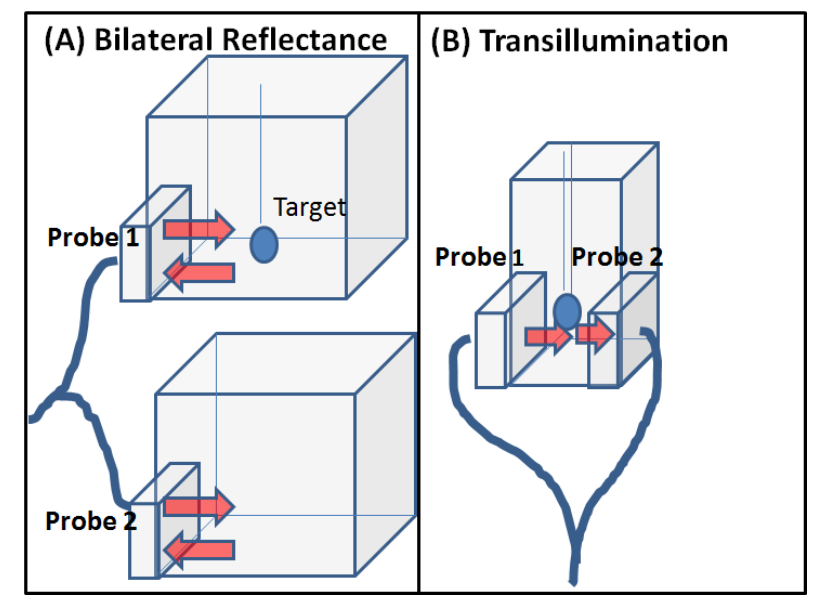

Figure 40: Schematic of experimental tissue phantom study set-up for (A) simultaneous bilateral reflectance imaging, and (B) transillumination imaging

The optical properties of 3\% Liposyn solution in terms of absorption $\left(\mu_{\mathrm{a}}\right)$ and reduced scattering $\left(\mu_{\mathrm{s}}{ }^{\prime}\right)$ coefficient are 0.09 and $9.5 \mathrm{~cm}^{-1}$, respectively, as measured using our single pixel homodyne optical imaging system. The typical optical properties of human breast tissues are $\mu_{\mathrm{a}}=0.04 \pm 0.02 \mathrm{~cm}^{-1}, \mu_{\mathrm{s}}^{\prime}=8 \pm 4 \mathrm{~cm}^{-1}$ [45-48]. Hence, the phantoms used in the current studies have similar optical properties of a human breast tissue. The absorption coefficient of $0.08 \%$ India Ink is $\sim 0.3 \mathrm{~cm}^{-1}$ as estimated by researchers in the past (at $750 \mathrm{~nm}$ wavelength) [28]. 
The reduced scattering coefficient $\left(\mu_{\mathrm{s}}^{\prime}\right)$ of India Ink can vary between 4 to $20 \mathrm{~cm}^{-1}$ as reported by various researchers [42-44], and was challenging to estimate in our studies. The optical properties of medium and target were used to perform simulations studies to determine maximum expected depth recovered. This information was used in the experimental design to limit the depth investigated.

The criteria for successful localization of the target were detailed in section 3.2 .9 (less than $1 \mathrm{~cm}$ distance from detected and true target location). The detected target location is found from the polynomial fitting of the intensity data as point of max intensity (from the curve). The maximum intensity point (centroid of the intensity distribution) is likely to be the center of the target demarcating the true location of the target within the medium. A cutoff value of $1 \mathrm{~cm}$ was chosen since the target diameter is $.95 \mathrm{~cm}$, and placement was subjected to human error for both target and probe placement. Experimental cases where the distance from detected target location and true location was less than $1 \mathrm{~cm}$ were found to have more accurate values of estimated target size and contrast. It is likely that distances between true and detected target locations less than 1 $\mathrm{cm}$ were subject to less noise (artifacts) and hence the resultant estimated target size and contrast were better localized. 
Table 11 is a portion of table with consistent experimental conditions allowing for comparison of reflectance and transillumination through the various depths tested.

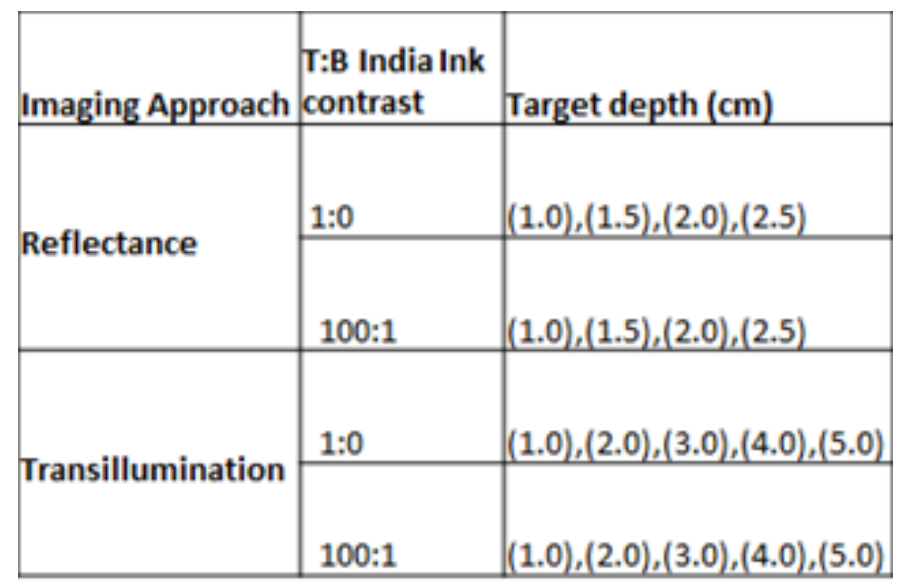

Table 11: Experimental cases for simultaneous bilateral reflectance and transillumination imaging studies. In all the studies 3\% Liposyn solution was used in the background phantoms and a 0.45 cc target was with $0.08 \%$ India Ink + 3\% Liposyn solution. In experimental studies with the a 100:1 contrast ratio the background contained $0.0008 \%$ India ink by volume.

\subsubsection{Data Analysis}

The experimental data is processed by subtraction (Reflectance- simultaneous bilateral) (Transillumination- subtraction with target removal), removal of detectors, normalization and polynomial fitting as explained in section 3.2.5.

\subsection{Results and Discussion}

Tables 9 and 10 show the experimental cases conducted where successful localizations (as describe in section 3.2.9) are shaded (in gray) (Metrics are for tables 9-10 are provided in Appendix D). Figures 41-46 provide 2D surface contour plots of the shallowest case and the deepest detected case (if possible the next unsuccessful case is also displayed). 


\section{Reflectance .8\% Target}

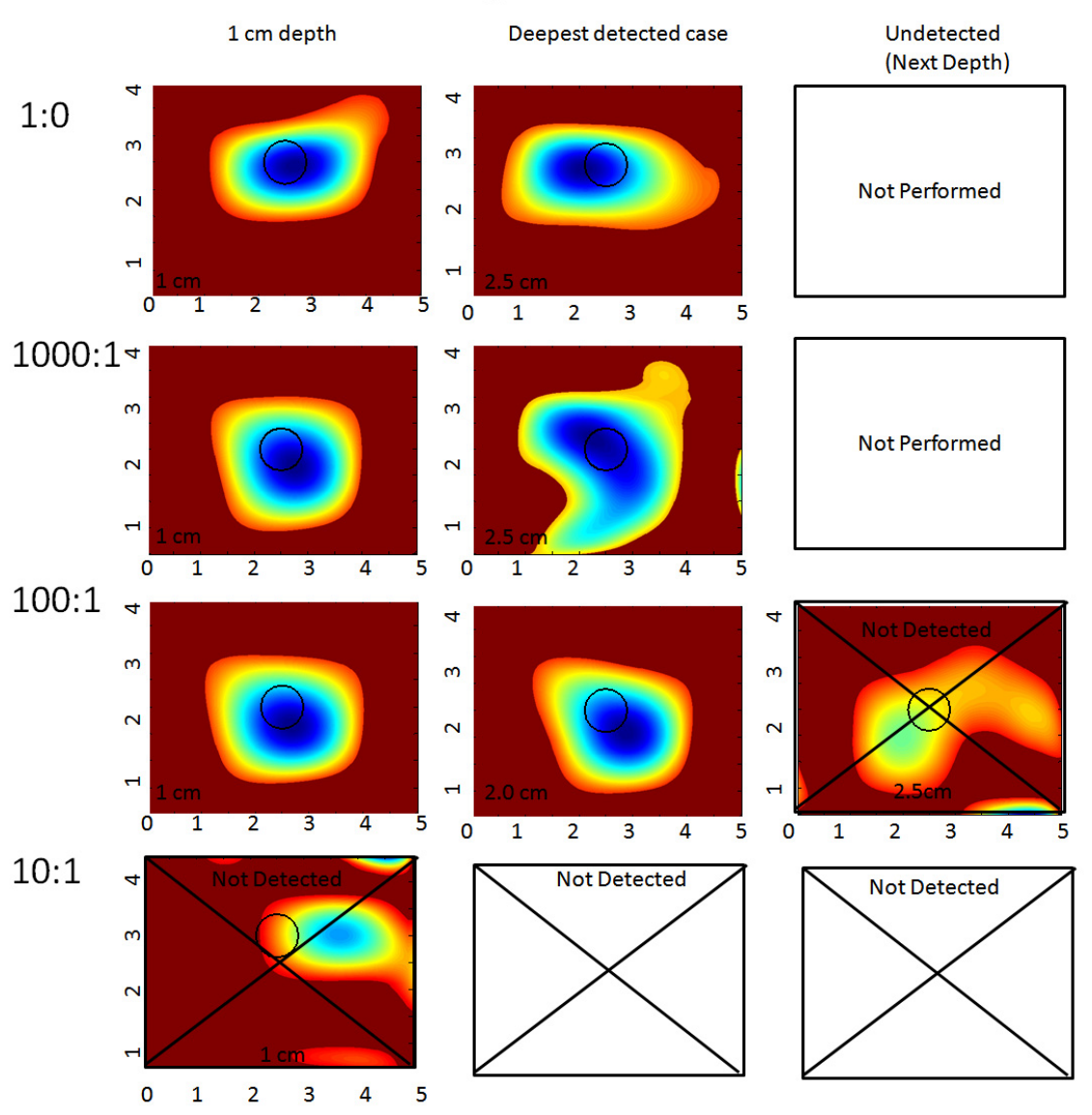

Figure 41: 2D surface contour plots (Reflectance imaging) for a $0.45 \mathrm{cc}$ spherical target filled with a $0.8 \%$ by volume India ink. The target was placed in a $10 \times 10 \times 10 \mathrm{~cm}^{3}$ acrylic liposyn phantom with a India ink to make the contrast between target and background 1:0,1000:1, 100:1 and 10:1.where the black circle represents true target location

From figure 41 it is seen that a $0.8 \%$ by volume India ink target provides high absorption that is localized at $2.5 \mathrm{~cm}(1: 0-$ Exp4a and 1000:1-Exp4b) and $2.0 \mathrm{~cm}(100: 1 \mathrm{Exp} 3 \mathrm{c})$ depth. The undetected case for 100:1 at $2.5 \mathrm{~cm}$ (Exp4c) displays an area of high absorption located just over $1 \mathrm{~cm}$ from the true target location which could have been due to error in placement of the target. At a target to background (T:B) of 10:1 no case resulted in the estimated target location at less than $1 \mathrm{~cm}$ than the true target location (considered unsuccessful localizations). 


\section{Reflectance $.08 \%$ Target}

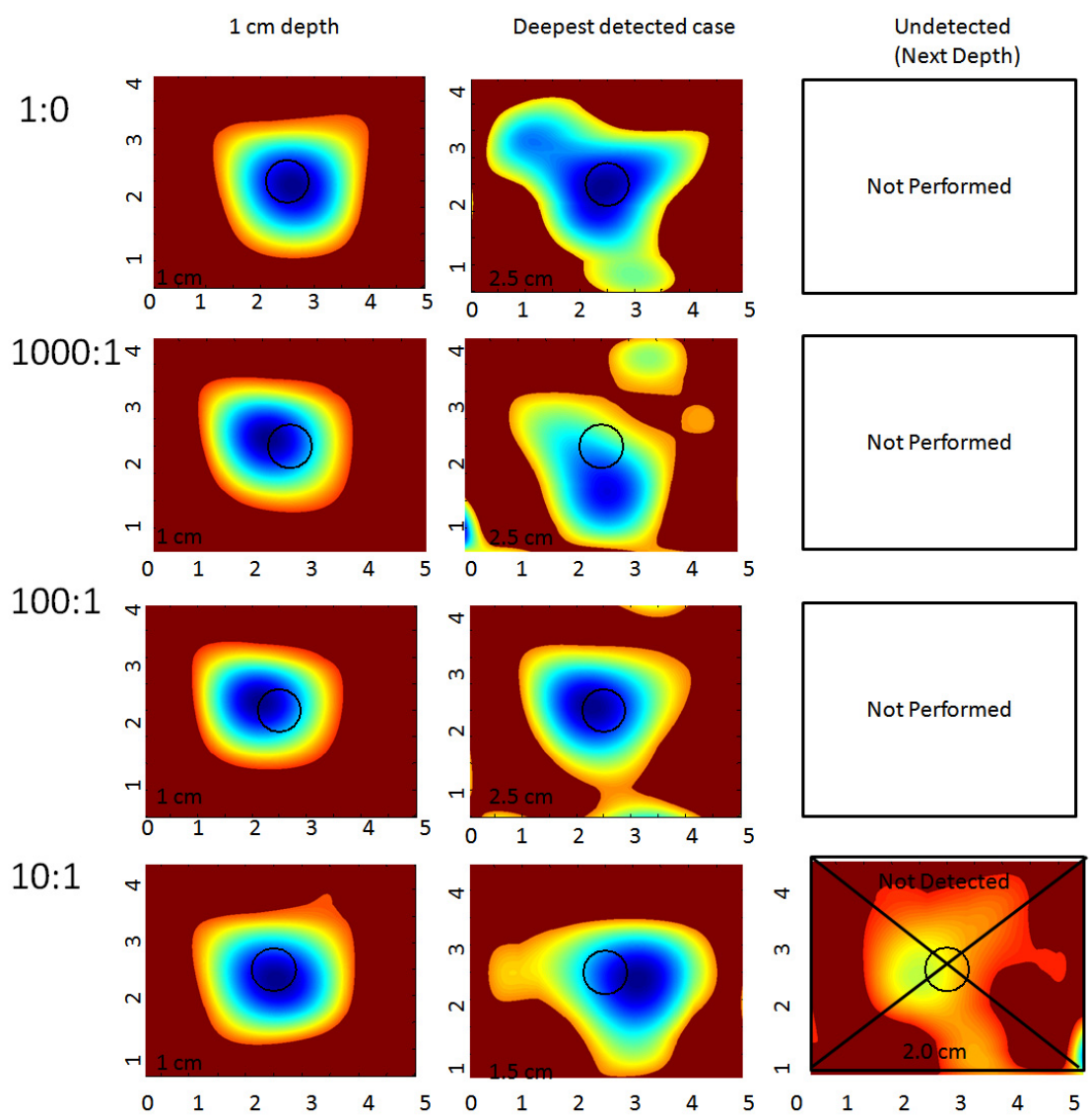

Figure 42: 2D surface contour plots (Reflectance imaging) for a $0.45 \mathrm{cc}$ spherical target filled with a $0.08 \%$ by volume India ink. The target was placed in a $10 \times 10 \times 10 \mathrm{~cm}^{3}$ acrylic liposyn phantom containing India ink making the $\mathrm{T}: \mathrm{B}$ contrast ratio 1:0,1000:1, 100:1 and 10:1.where the black circle represents true target location

From figure 42 it is seen that a $0.08 \%$ by volume India ink target provides high absorption and is recovered up to $2.5 \mathrm{~cm}$ target depth [1:0, 1000:1 and 100:1 $\operatorname{Exp}(5-8)(\mathrm{e} . \mathrm{f}$ and g)] (no cases beyond $2.5 \mathrm{~cm}$ were performed). With a $0.08 \%$ target, $10: 1$ contrast ratio is detectible up to 1.5 $\mathrm{cm}$ (Exp6h) unlike the $0.8 \%$ target [10:1 $\operatorname{Exp}(1-4) \mathrm{d}]$ (not successful) the other cases were not localized within $1 \mathrm{~cm}$ of true target location. Experimental case [10:1 Exp7h] $(0.08 \%)$ has the 
point of maximum absorption located on the outer detectors possible due to loss of contact between target and background images due to human error.

\section{Reflectance $.008 \%$ Target}

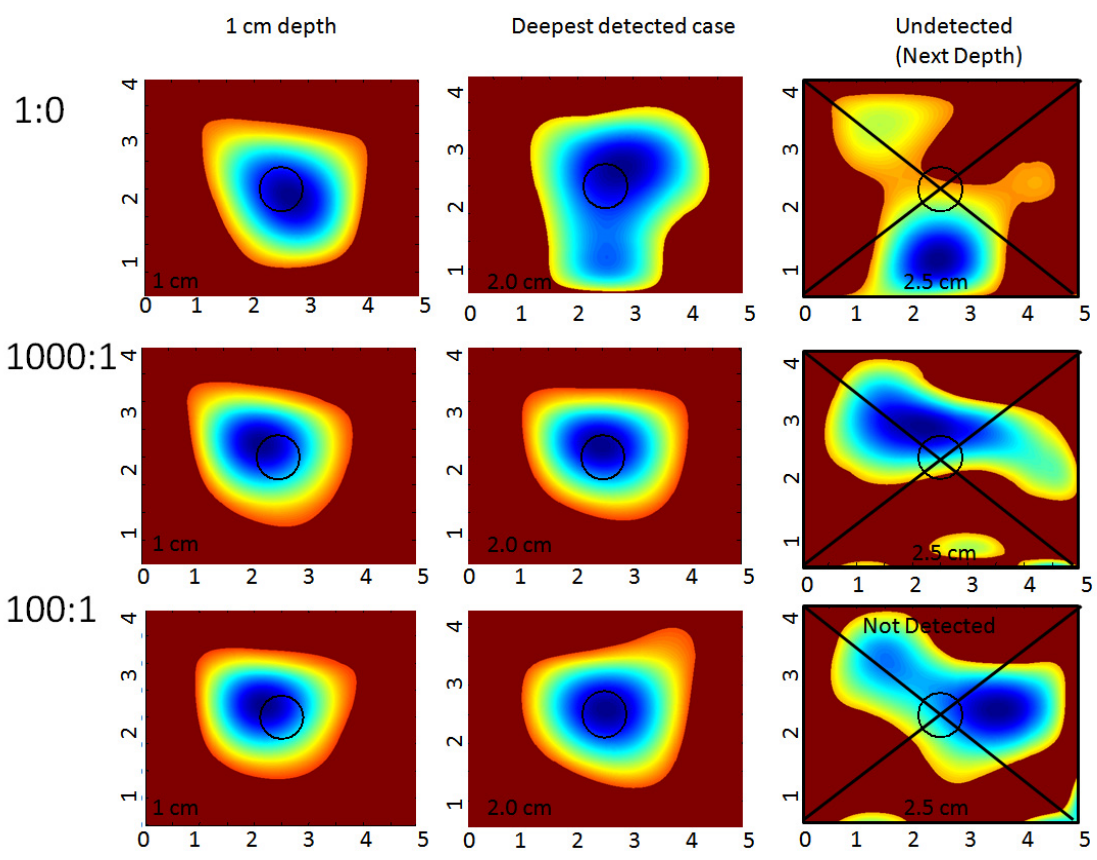

$10: 1$
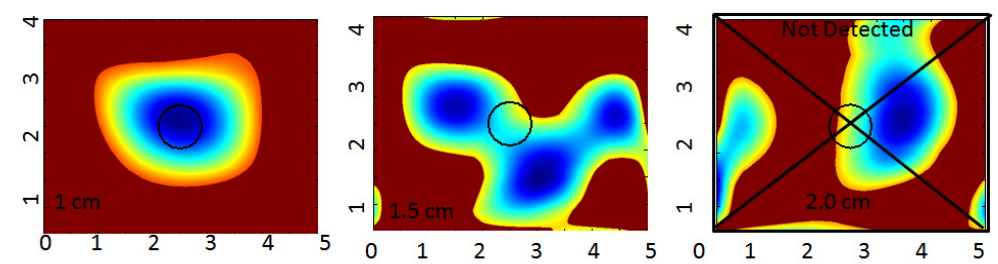

Figure 43: 2D surface contour plots (Reflectance imaging) for a $0.45 \mathrm{cc}$ spherical target filled with a $0.008 \%$ by volume India ink. The target was placed in a $10 \times 10 \times 10 \mathrm{~cm}^{3}$ acrylic liposyn phantom containing India ink making the $\mathrm{T}: \mathrm{B}$ contrast ratio 1:0, 1000:1, 100:1 and 10:1.where the black circle represents true target location

From figure 43 it is seen that a $0.008 \%$ is not localize at a depth of $2.5 \mathrm{~cm}[1: 0,1000: 1,100: 1$ and 10:1 $(\operatorname{Exp}(12)(\mathrm{i}, \mathrm{j}, \mathrm{k}$ and 1)] likely due to insufficient absorption from the target. Experimental case $[10: 1 \operatorname{Exp}(9-12) 1](0.008 \%)$ are not localized and provided insight as to why [10:1 $\operatorname{Exp}(1-$ 4)d] $(0.8 \%)$ was not localized successfully. A $0.008 \%$ target at a $\mathrm{T}: \mathrm{B}$ of 10:1 has much less 
absorption in the background than a 0.8 target at a $\mathrm{T}: \mathrm{B}$ of $10: 1$. It is likely that the absorption of the medium for a $0.8 \%$ target is too high (T:B $10: 1)$.

\section{Transillumination .8 \% Target}

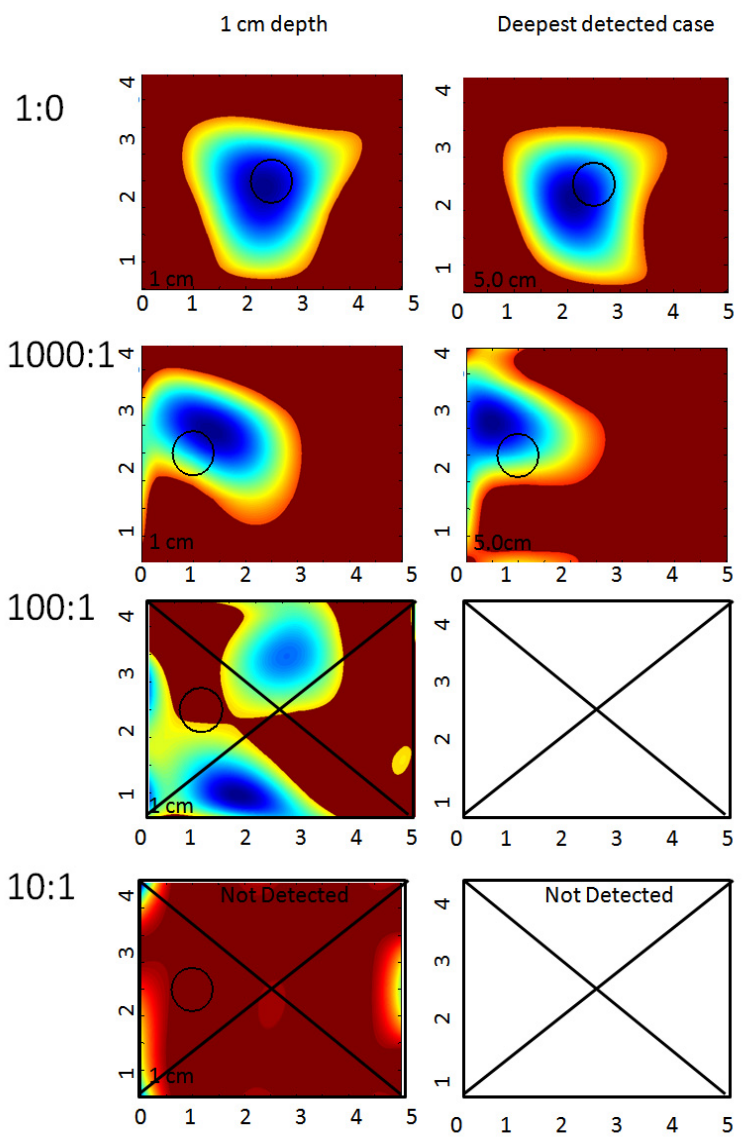

Figure 44: 2D surface contour plots (Transillumination imaging) for a 0.45 cc spherical target filled with a $0.8 \%$ by volume India ink. The target was placed in a $10 \times 10 \times 10 \mathrm{~cm}^{3}$ acrylic liposyn phantom containing India ink making the T:B contrast ratio 1:0, 1000:1, 100:1 and 10:1.where the black circle represents true target location.

From figure 44 it is seen that for transillumination imaging as supported by [1:0 and 1000:1 $\operatorname{Exp}(1-5)(\mathrm{a}, \mathrm{b})](0.8 \%)($ detected) and $[100: 1$ and $10: 1 \operatorname{Exp}(1-5)(\mathrm{c}, \mathrm{d})]$ is either detected at all depths $(1-5 \mathrm{~cm})$ or not detected at any. From [100:1 $\operatorname{Exp}(1-5) \mathrm{c}]$ and $[10: 1 \operatorname{Exp}(1-5) \mathrm{d}](0.8 \%)$ it is seen that the targets are not localized which represents the same occurrence in 
transillumination as in reflectance [10:1 $\operatorname{Exp}(1-4) \mathrm{d}]$ where the absorption in the background prevent sufficient light from reaching the detectors.

\section{Transillumination .08\% Target}

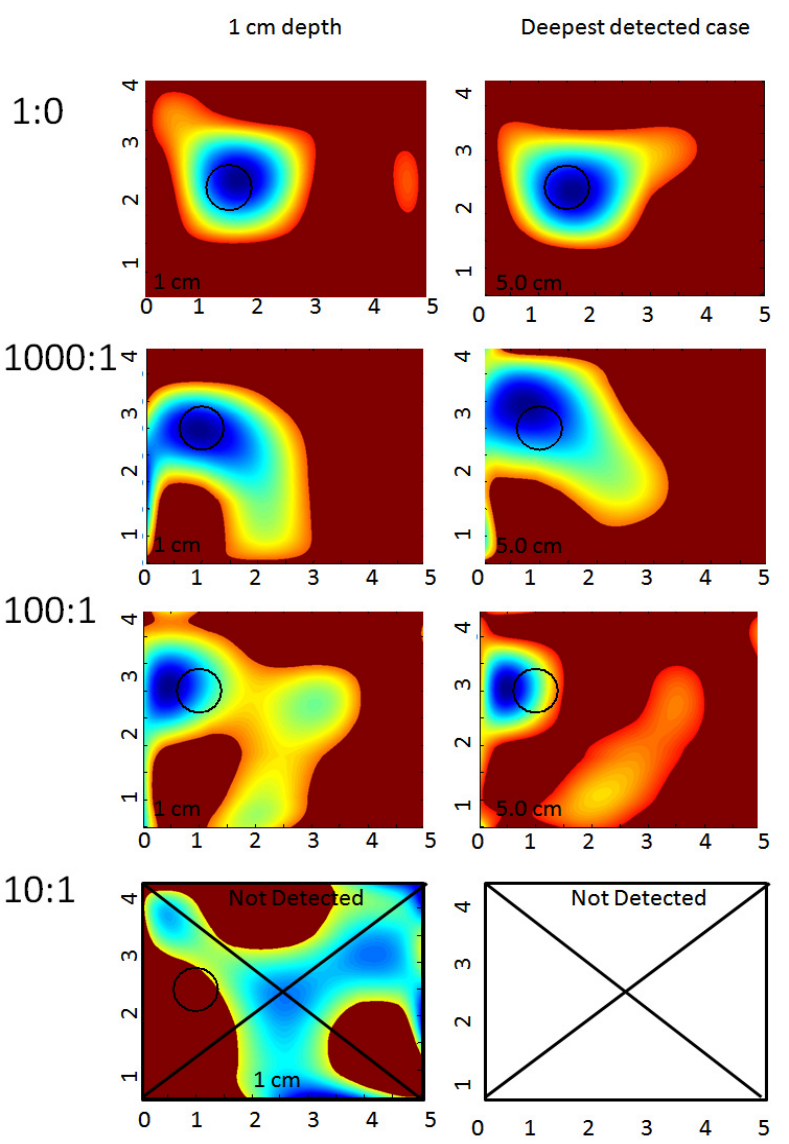

Figure 45: 2D surface contour plots (Transillumination imaging) for a $0.45 \mathrm{cc}$ spherical target filled with a $0.08 \%$ by volume India ink. The target was placed in a $10 \times 10 \times 10 \mathrm{~cm}^{3}$ acrylic liposyn phantom containing India ink making the $\mathrm{T}: \mathrm{B}$ contrast ratio 1:0, 1000:1, 100:1 and 10:1.where the black circle represents true target location.

From figure 45 it can be observed that at a contrast ratio of $[100: 1 \operatorname{Exp}(6-10) \mathrm{g}](0.08 \%)$ is detected which is expected as reflectance imaging of a [100:1 $\operatorname{Exp}(5-9) \mathrm{g}](0.08 \%)$ contrast ratio. A contrast ratio of $[10: 1(\operatorname{Exp}(6-10) \mathrm{h})](0.08 \%)$ is not localized within $1 \mathrm{~cm}$ and resulting 2D 
surface plot shows a region of high absorption in the middle possibly due to artifact generation due to low light signal reaching the detector.

\section{Transillumination $.008 \%$ Target}
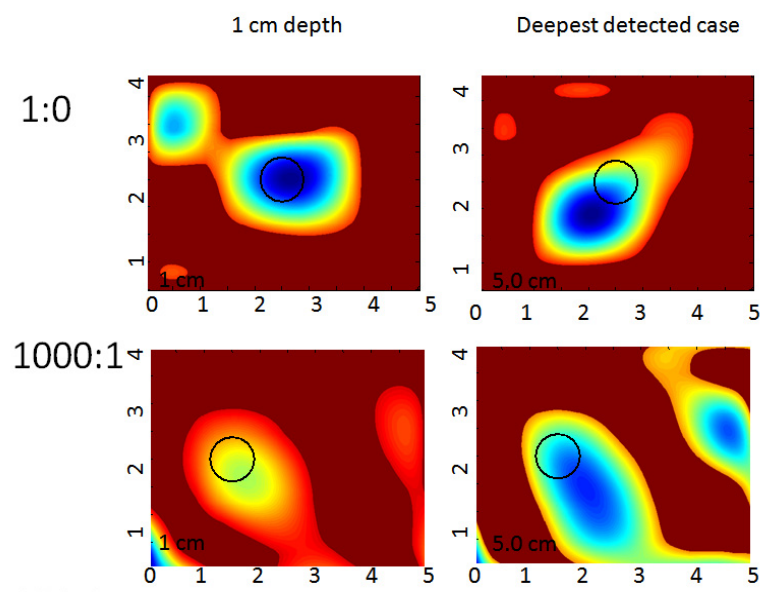

100:1
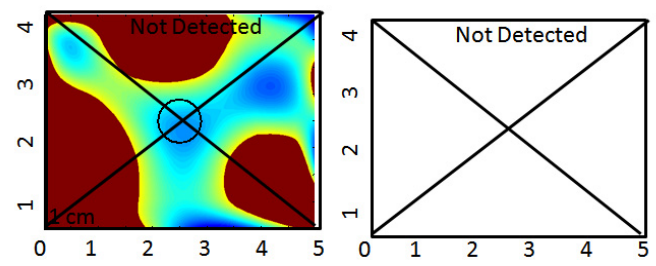

10:1

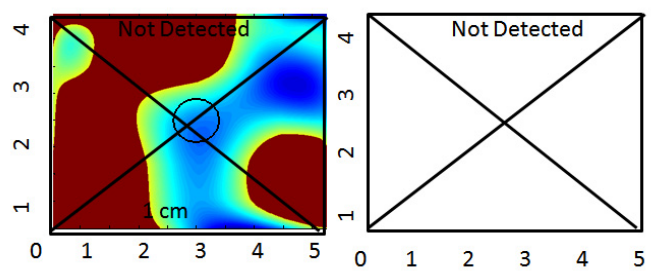

Figure 46: 2D surface contour plots (Transillumination imaging) for a $0.45 \mathrm{cc}$ spherical target filled with a $0.008 \%$ by volume India ink. The target was placed in a $10 \times 10 \times 10 \mathrm{~cm}^{3}$ acrylic liposyn phantom containing India ink making the T:B contrast ratio 1:0, 1000:1, 100:1 and 10:1.where the black circle represents true target location..

Figure 46 shows that for a $0.008 \%$ (India ink by volume) successful localization occurs in two experimental cases [1:0 and 1000:1 $\operatorname{Exp}(11-15)(\mathrm{i}$ and $\mathrm{j})](0.008 \%)$. At 1:0 and 1000:1 the target was localized through the entire depth $(1-5 \mathrm{~cm}$ container length) where for reflectance at 1:0 and 1000:1 $(0.008 \%)$ at $2.5 \mathrm{~cm}$ localization did not occur within $1 \mathrm{~cm}$ distance from true location 
[1:0 and 1000:1 Exp12(i,j,k and 1)] (0.008\%). Experimental cases [100:1 and 10:1 Exp(11-15)(k andl)] $(0.008 \%)$ were both not localized.

Based on the findings of the experimental studies several inferences can be drawn for both reflectance and transillumination. The most apparent inference is that transillumination imaging allows the localization of targets at deeper maximum depths than reflectance. Also apparent is that if the target is detectable at any depth in a transillumination study it will be localized at all depths (table 9), which is not true for reflectance. However, reflectance imaging has more cases where localization was possible than transillumination and becomes disproportionate at lower target to background ratios (T:B) (i.e. reflectance imaging has higher success at lower T:B ratios with shallow targets). The difference is expected since reflected light at shallow target positions have a shorter travel distance when compared with transilluminated light, which must travel the entire distance of the phantom regardless of target position. Transillumination appears to be more restrictive with regards to the target to background $(\mathrm{T}: \mathrm{B})$ contrast than reflectance imaging since highly absorbing backgrounds prevent transmission of light. In experimental cases [1:0 $(\operatorname{Exp}(1-5) \mathrm{a})$ and $1000: 1(\operatorname{Exp}(1-5) \mathrm{b})](0.8 \%)$ the target was localized within $1 \mathrm{~cm}$ while in experimental cases [100:1 and 10:1 $\operatorname{Exp}(1-5)(\mathrm{c}$ and d)] $(0.8 \%)$ it was not. Furthermore reducing the absorption of the background such as with a $0.08 \%$ target in [100:1 Exp(1-5)g] localization is successful. Reflectance imaging follows similar trends as transillumination imaging such as in experimental set $(\operatorname{Exp}(1-4) \mathrm{d})$ a target is not localized due to high absorption at lower $\mathrm{T}: \mathrm{B}$ contrast ratios. The effect is due to the background being too highly absorbing preventing signal from reaching the detector. Whereas, when $0.008 \%$ by volume target is employed (transillumination) detection does not occur at $\mathrm{T}: \mathrm{B}$ ratios lower than 1:0 $\operatorname{Exp}(11-15) \mathrm{i}$. Comparable examples in reflectance would be [Exp7h, $\operatorname{Exp} 8(f, g$ and $h)-0.08]$, [Exp111, 
Exp12(i,j,k and 1)- 0.008 ]. The inability to detect targets at $\mathrm{T}: \mathrm{B}$ ratios lower than $1: 0$ with a $0.008 \%$ target is likely due to insufficient T:B contrast through the length of the tissue (i.e. the medium has more absorption through the length than the target). Hence, experimental data supports that transillumination provides deeper target detection than reflectance imaging only when the $\mathrm{T}: \mathrm{B}$ contrast is substantially large and the absorption of the background is sufficiently low to allow transmission of light. It is possible for reflectance imaging to localize targets at shallow depths in highly absorbing media where transillumination would fail to localize a target.

\subsubsection{Comparison of reflectance vs. transillumination: Reflectance}

Figure 47 shows the 2D surface contour plots (via polynomial fit) of the reflected (and subtracted) intensity signals obtained from a $0.45 \mathrm{cc}$ target placed at various depths across the $10 \times 10 \times 10 \mathrm{~cm}^{3}$ phantom. Each experimental case was normalized since the instrumentation settings were different for each depth and T:B contrast ratio cases. From the plots, it is obvious that the target was detectable up to $2.5 \mathrm{~cm}$ deep, at both the chosen T:B (India Ink) contrast ratios. At depths of $3 \mathrm{~cm}$ and higher, the targets were not detectable in simulation studies and hence not imaged (Appendix D). The measurement errors were consistently $\sim 0.2$ across any given target depth (up to $2.5 \mathrm{~cm}$ ). The target was localized closer to its true location in all cases. The detected target location, target size, measurement errors and T:B detected contrast are given in Table 12 (Appendix D contains the full metrics table for all reflectance studies ). 


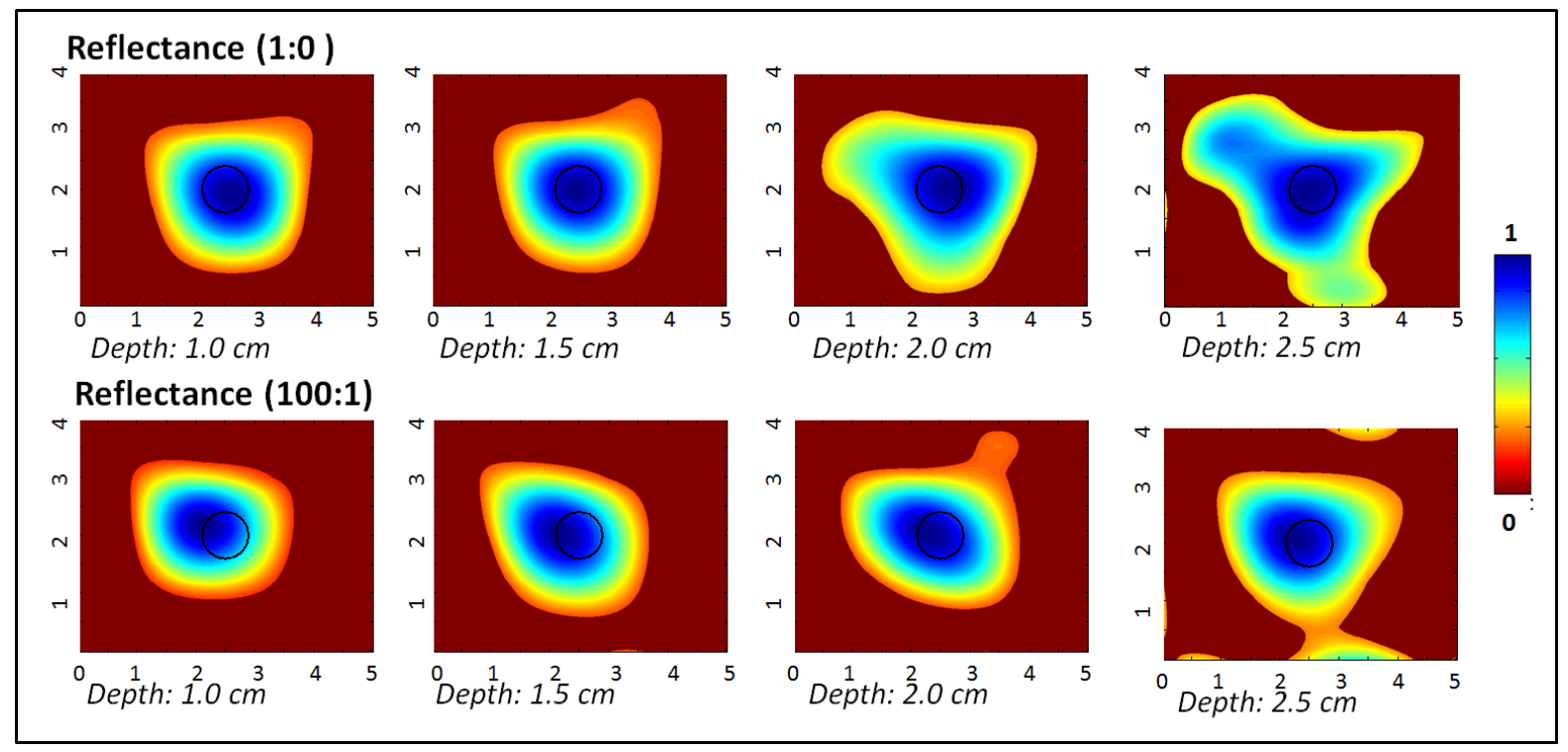

Figure 47: Two-dimensional surface contour plots (via 5th degree polynomial fit) of reflectance imaging studies of a $0.45 \mathrm{cc}$ spherical target at 1:0 and 100:1 T:B India Ink contrast, located at various depths. The black hollow circle represents the true $2 \mathrm{D}$ target location.

\begin{tabular}{|c|c|c|c|c|c|c|c|}
\hline $\begin{array}{l}\text { Contrast } \\
\text { ratio (India } \\
\text { Ink) }\end{array}$ & $\begin{array}{c}\text { True target } \\
\text { location } \\
{[\mathrm{x}, \mathrm{y}](\mathrm{cm})}\end{array}$ & $\begin{array}{c}\text { True target } \\
\text { depth, z } \\
\text { (cm) }\end{array}$ & $\begin{array}{l}\text { Detected } \\
\text { Location } \\
{[\mathrm{x}, \mathrm{y}](\mathrm{cm})}\end{array}$ & $\begin{array}{c}\text { Distance off } \\
\text { from true } \\
{[x, y] \text { location }} \\
\text { (cm) }\end{array}$ & $\begin{array}{l}\text { Recovered } \\
T: B \text { contrast }\end{array}$ & $\begin{array}{c}\text { Estimated } \\
\text { target } \\
\text { diameter } \\
\text { (cm) }\end{array}$ & $\begin{array}{c}\text { Measurement } \\
\text { error }\end{array}$ \\
\hline \multirow{4}{*}{$1: 0$} & \multirow{4}{*}[2.5,2]{} & 1 & {$[2.59,1.92]$} & 0.12 & 8.3 & 1.88 & 0.218 \\
\hline & & 1.5 & {$[2.47,2]$} & 0.03 & 7.4 & 1.94 & 0.216 \\
\hline & & 2 & {$[2.7,2.02]$} & 0.20 & 4.3 & 0.78 & 0.218 \\
\hline & & 2.5 & {$[2.46,1.9]$} & 0.11 & 12.5 & 0.94 & 0.221 \\
\hline \multirow{4}{*}{$100: 1$} & \multirow{4}{*}[2.5,2]{} & 1 & {$[2.12,2.16]$} & 0.41 & 8.8 & 1.53 & 0.211 \\
\hline & & 1.5 & {$[2.14,2.15]$} & 0.39 & 8.2 & 1.62 & 0.211 \\
\hline & & 2 & {$[2.15,2.14]$} & 0.38 & 9.3 & 1.54 & 0.211 \\
\hline & & 2.5 & $(2.27,2.08)$ & 0.24 & 5.0 & 1.62 & 0.218 \\
\hline
\end{tabular}

Table 12: Table 2. Quantitative details of the detected target location, size, recovered T:B contrast and measurement errors for different experimental cases using the Gen-2 imager towards reflectance imaging studies. 


\subsubsection{Comparison of reflectance vs transillumination: Transillumination}

Figure 48 shows the 2D surface contour plots (via polynomial fit) of the transilluminated (and subtracted) intensity signals obtained from a $0.45 \mathrm{cc}$ target placed at various depths across the $10 \times 10 \times 10 \mathrm{~cm}^{3}$ phantom. From the plots, it is obvious that the target was detectable across the entire depth of the phantom (i.e. $5 \mathrm{~cm}$ deep), at both the chosen optical contrast ratios. The target was localized closer to its true location in all cases ( $\leq 0.5 \mathrm{~cm}$ in most cases), although there were obvious artifacts without the data post-processing techniques. The measurement errors were two orders of magnitude smaller for 1:0 contrast cases in comparison to the 100:1 contrast cases. The detected target location, target size, measurement errors and $\mathrm{T}: \mathrm{B}$ detected contrast are given in Table 13 (Appendix D contains the full metrics table for all transillumination studies ).

The progression of images exhibit that as the target is closer to the detection surface (i.e. further away from the illumination surface), where the optical detection fibers are placed a reduction in artifacts is visible, as well as an improvement in location estimation. Transillumination measurements in General provide greater depth information of the tissues over reflectance measurements. Typically most studies have focused on using single point source illumination to demonstrate the effectiveness of transillumination measurements. The current studies are different in terms of the source illumination configuration. Unlike the typically used sequential point illumination employed by other researchers, the Gen-2 hand-held imager can perform simultaneous multiple point illumination towards enhanced imaging rates. Thus, even simultaneous illumination, which can have null (intensity) points from interferences, can still detect deep targets based via transillumination imaging over reflectance, independent of the illumination configuration. 


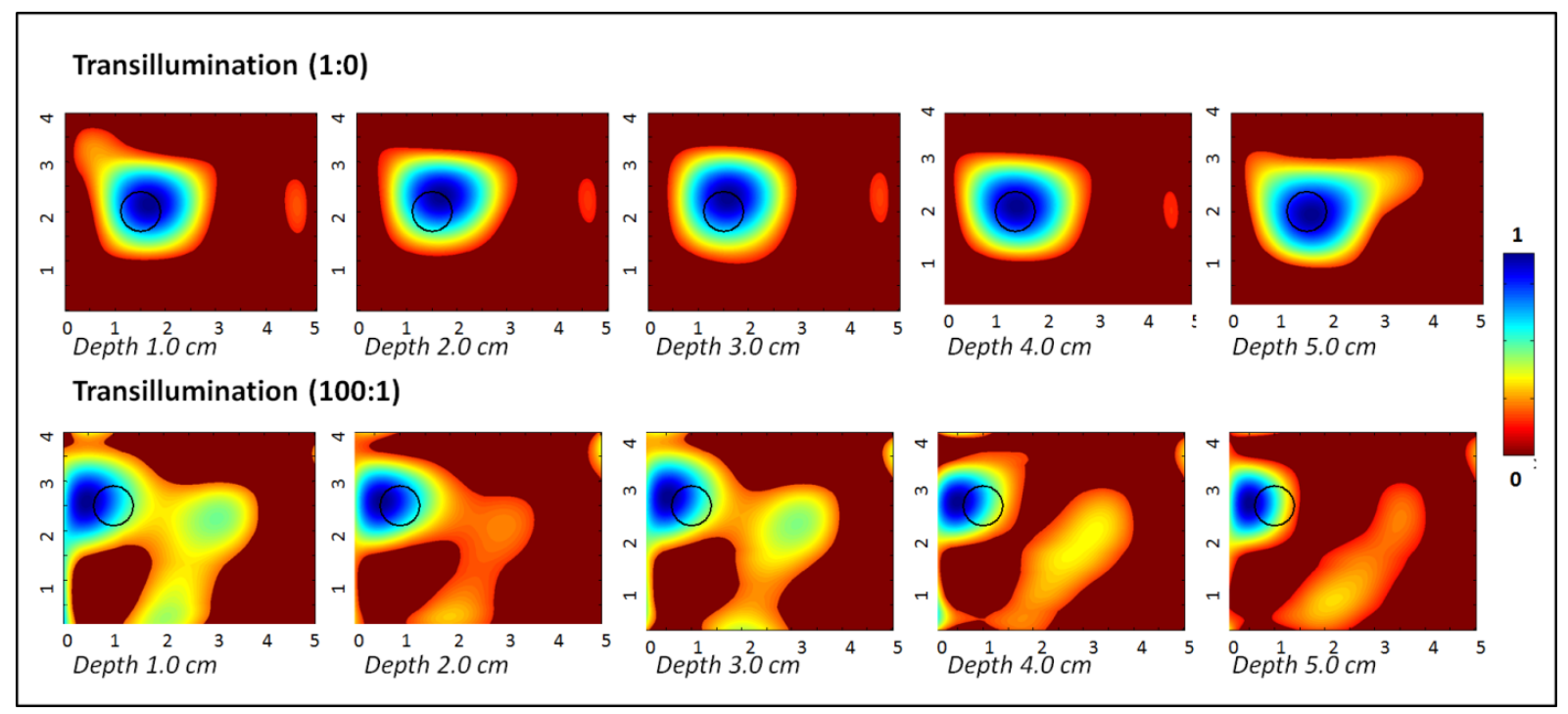

Figure 48: Two-dimensional surface contour plots (via 5th degree polynomial fit) of transilluminated reflectance imaging studies of a $0.45 \mathrm{cc}$ spherical target at 1:0 and 100:1 T:B India Ink contrast, located at various depths. The black hollow circle represents the true 2D target location.

\begin{tabular}{|c|c|c|c|c|c|c|c|}
\hline $\begin{array}{c}\text { Contrast } \\
\text { ratio } \\
\text { (India Ink) }\end{array}$ & $\begin{array}{c}\text { True } \\
\text { target } \\
\text { location } \\
{[\mathrm{x}, \mathrm{y}](\mathrm{cm})}\end{array}$ & $\begin{array}{l}\text { True target } \\
\text { depth, } \mathrm{z}(\mathrm{cm})\end{array}$ & $\begin{array}{c}\text { Detected } \\
\text { Location }[\mathrm{x}, \mathrm{y}] \\
(\mathrm{cm})\end{array}$ & $\begin{array}{l}\text { Distance off } \\
\text { from true } \\
\text { [x,y] } \\
\text { location } \\
\text { (cm) }\end{array}$ & $\begin{array}{c}\text { Recovered } \\
\mathrm{T}: \mathrm{B} \\
\text { contrast }\end{array}$ & $\begin{array}{c}\text { Estimated } \\
\text { target } \\
\text { diameter } \\
(\mathrm{cm})\end{array}$ & $\begin{array}{c}\text { Measurement } \\
\text { error }\end{array}$ \\
\hline \multirow{5}{*}{ 1:0 } & \multirow{5}{*}[1.5,2]{} & 1 & {$[1.58,2.4]$} & 0.41 & 19.286 & 1.70 & 0.0009 \\
\hline & & 2 & {$[1.63,2.43]$} & 0.45 & 19.294 & 0.86 & 0.0005 \\
\hline & & 3 & {$[1.59,2.44]$} & 0.45 & 17.773 & 0.96 & 0.0007 \\
\hline & & 4 & {$[1.55,2.35]$} & 0.35 & 17.731 & 0.88 & 0.0007 \\
\hline & & 5 & {$[1.65,2.04]$} & 0.15 & 19.727 & 0.42 & 0.0016 \\
\hline \multirow{5}{*}{$100: 1$} & \multirow{5}{*}[1,2.5]{} & 1 & {$[0.55,2.82]$} & 0.55 & 3.762 & 1.24 & 0.252 \\
\hline & & 2 & {$[0.63,2.79]$} & 0.47 & 4.382 & 1.14 & 0.245 \\
\hline & & 3 & {$[0.61,2.82]$} & 0.50 & 3.947 & 0.95 & 0.241 \\
\hline & & 4 & {$[0.6,2.85]$} & 0.53 & 4.265 & 0.97 & 0.248 \\
\hline & & 5 & {$[0.57,2.74]$} & 0.49 & 5.756 & 0.74 & 0.241 \\
\hline
\end{tabular}

Table 13: Quantitative details of the detected target location, size, recovered T:B contrast and measurement errors for different experimental cases using the Gen-2 imager towards transillumination imaging studies. 


\subsection{Conclusions}

In this study, the feasibility of reflectance and transillumination diffuse optical imaging was demonstrated on tissue phantoms. During reflectance imaging targets up to $2.5 \mathrm{~cm}$ deep were localized from 2D surface images. During transillumination imaging, targets up to $5 \mathrm{~cm}$ deep were localized. It can be observed from these experimental cases that transillumination provides a greater target depth recovery than reflectance imaging. It was also observed that transilluminated signals get weaker when the target is further away from the detected surface (or closer to the illumination surface), especially as $\mathrm{T}: \mathrm{B}$ contrast ratio reduces (table 9). The unique capability of the Gen-2 hand-held optical imager to perform both reflectance and transillumination imaging is an advantage. For instance a target closer to a surface can be detected by reflectance even at lower $\mathrm{T}: \mathrm{B}$ contrast ratios, at places where transillumination can be limited by tissue volume or depth. Also flexibility to illuminate one or more point sources simultaneously allows for enhance imaging rates while still detecting deeper targets 
CHAPTER 6: Reflectance and Transillumination imaging of curved tissue phantoms

\subsection{Introduction}

The Gen-2 imager was developed as a 5 plate design towards imaging of human breast tissue which contained curved tissue geometries with low rigidity. The flexibility of the Gen-2 imaging probe allows the probe to contour curved geometries without applying compression. Compression would change hemodynamic properties within the diseased tissue thus lowering the endogenous tissue contrast associated with a highly vascular tumor. The focus of this chapter is to demonstrate the feasibility of performing hand-held optical imaging (reflectance and transillumination) on curved geometries which would facilitate implementation of the Gen-2 hand-held imager on human breast tissue (see section 7.1).

\subsection{Materials and Methods}

\subsubsection{Instrumentation}

The instrumentation associated with the Gen-2 imager is found in section (3.1) where the sources, probe and detector are discussed in detail.

\subsubsection{Experimental Studies}

Diffuse optical imaging experiments were performed with a gel phantom, applying both reflection and transillumination imaging (Figure 49). For reflectance a single probe was employed and placed on the curved surface over the approximate target location (target location is discussed further below). For transillumination both probes were employed where the source probe was placed on the underneath (flat) portion of the phantom, while the detection probe was placed over the curved surface (similar to reflectance). 


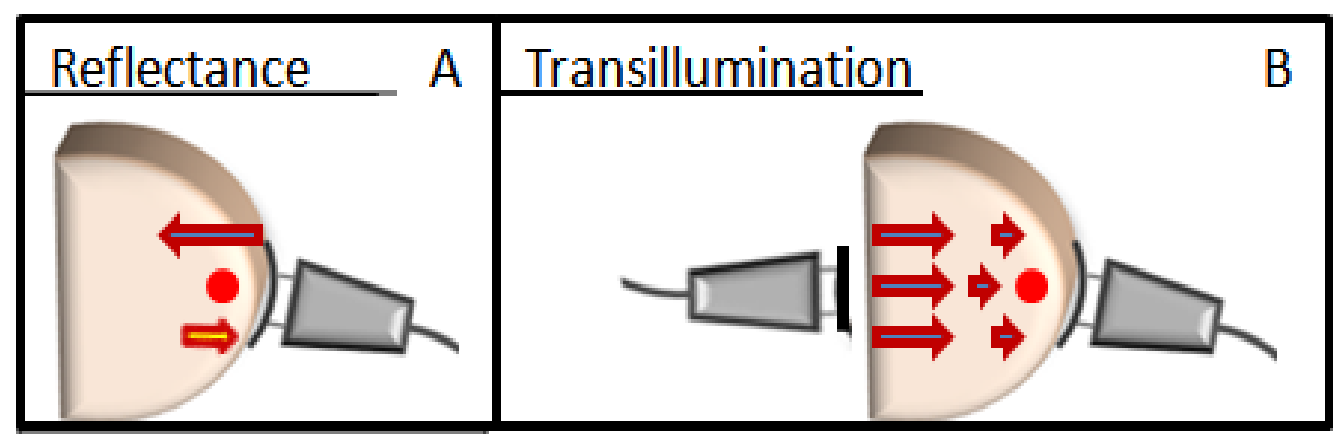

Figure 49: Diagram of probe placement during the scanning of a gel phantom with reflectance (A) and transillumination (B) imaging. The target location was approximately $1 \mathrm{~cm}$ off from the curved surface, where the arrows indicate the direction of illumination (long arrows) and light collection (short arrows).

The gel phantom was a mix of agar, liposyn and preservative in specific concentration solidified in a mold (major axis- $16 \mathrm{~cm}$, minor axis- $11.5 \mathrm{~cm}$, volume of $\sim 700 \mathrm{ml}$ ) to yield similar shape with optical properties resembling breast tissue as discussed by Grabel, et al ([36-37]) (Figure 50). A description of the phantom composition and creation is included in appendix E,F as an excerpt from another masters students thesis whose work had generated the phantom ([47]).

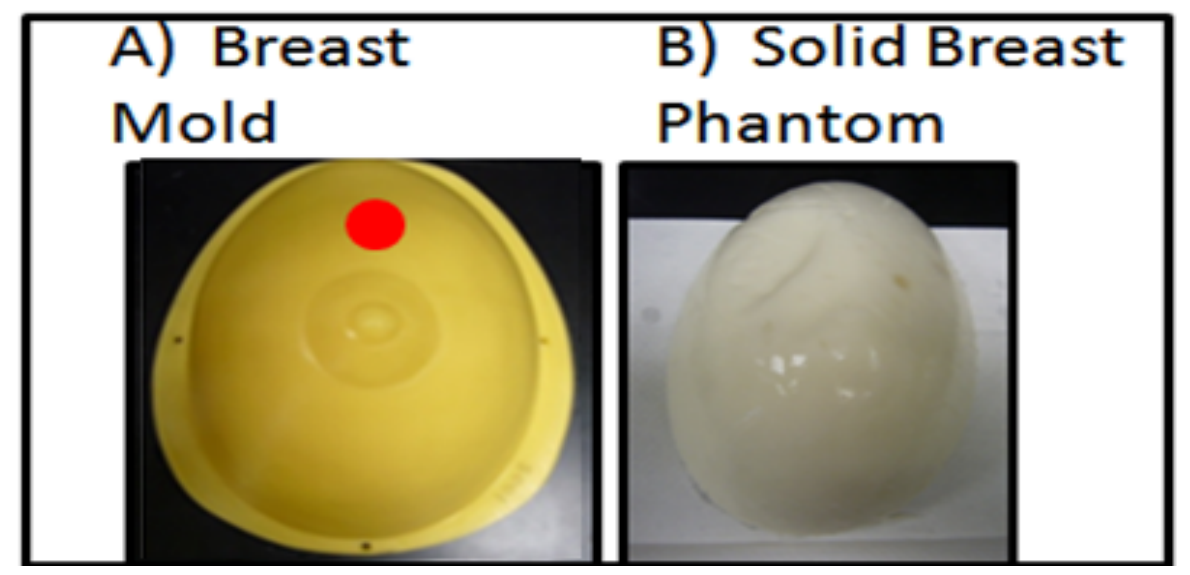

Figure 50: The breast mold (A) employed to produce a solid breast phantom (C) which has a major axis of $16 \mathrm{~cm}$ and a minor axis of $11.5 \mathrm{~cm}$ with a total volume of $\sim 700 \mathrm{ml}$. A $0.45 \mathrm{cc}$ India ink target was place $\sim 1 \mathrm{~cm}$ off the surface represented by the red circle. 
A $0.45 \mathrm{cc}$ India ink target was embedded in the gel phantom approximately $1 \mathrm{~cm}$ from the curved surface at the 12 o'clock position as seen on figure 50(A). The target is placed in the phantom prior to solidifying and hence the true target location is unknown, although it is estimated to be near the region indicated by the red circle (Figure 50A). Imaging involved progressive placement of the probe as to localize the target moving from left to right for transillumination and bottom to top for reflectance based measurements labeled as scans, where each scan is approximately 1 to $2 \mathrm{~cm}$ movement initial placement. Hence the absorption due to the target is expected to move in the opposite direction of scanning. In the case of reflectance imaging the direction of scanning was top to bottom as shown in figure 51. For transillumination imaging the direction of scanning was from left to right as shown in figure 52 .

\subsubsection{Data Processing}

The experimental data is processed by subtraction, removal of detectors nearest the sources and polynomial fitting as was explained in section 3.2 for both reflectance (single probe imaging) and transillumination imaging. The background image was acquired by imaging a region of the phantom (region of the same phantom far away where no target was located) where no target was present.

\subsection{Results and Discussion}

\subsubsection{Reflectance}

Figure 51 shows the 2D surface contour plots of the processed optical signal collected employing reflectance imaging from the gel phantom. In figure 51 a region of high absorption is shown moving in the opposite direction of scanning (top to bottom), because there is no other signal, it is unlikely to be due to artifacts generated during imaging. Since the true target location is unknown the estimated location is shown as a dotted circle. No calculations were performed to 
find the estimated target location or other metrics since the true target location would have been subjective. Viewing the images as sequential scans a region of high absorption appears to be moving as what is expected from the target embedded in the gel phantom. The region of high absorption is due to the target being successfully imaged in a gel phantom at a depth of $1 \mathrm{~cm}$ employing reflectance imaging with the Gen-2 hand-held optical imager.

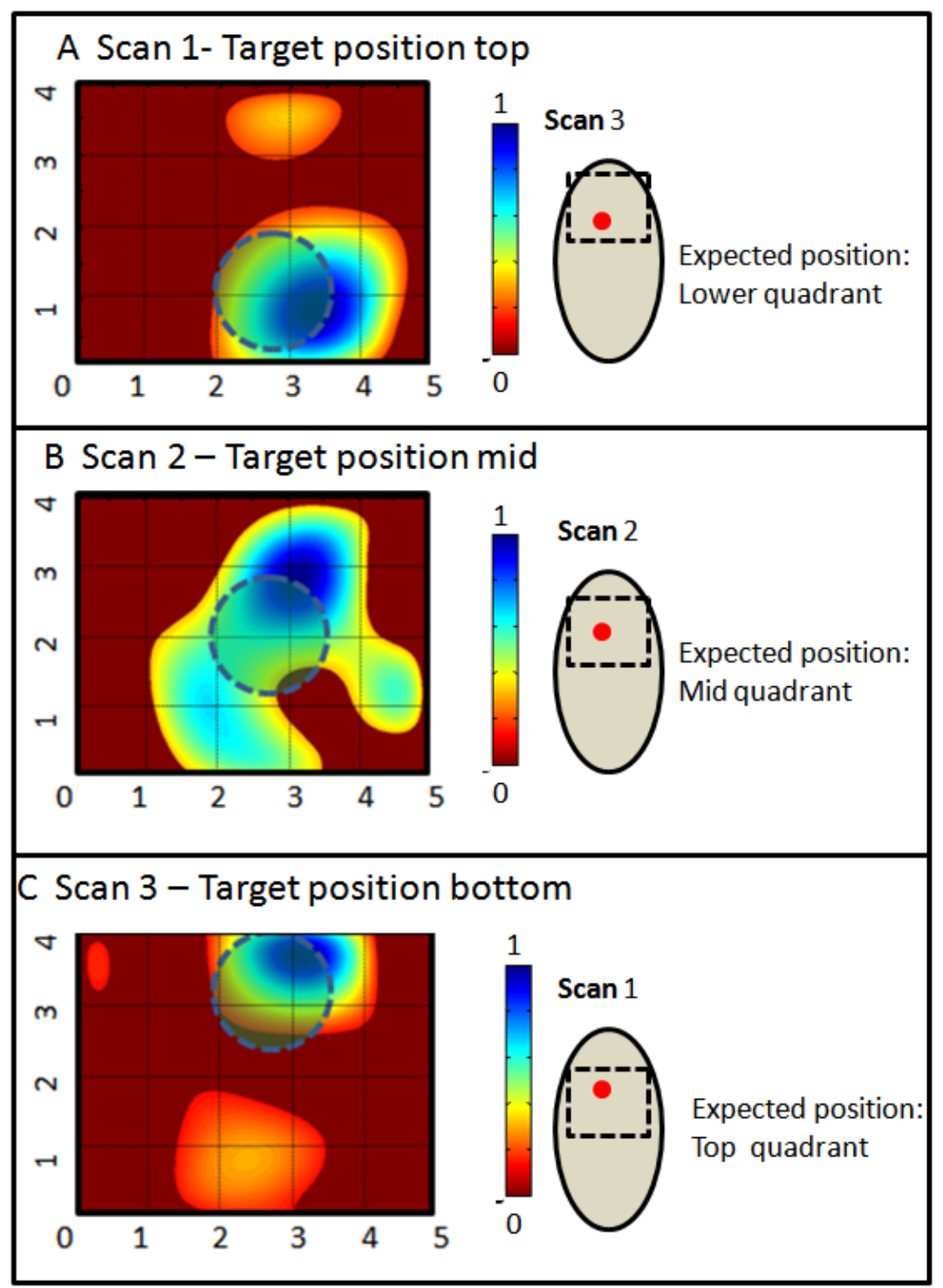

Figure 51: Single probe reflectance imaging was performed on a gel phantom with shape and optical properties resembling human breast tissue. A 0.45 cc India ink target $0.08 \%$ by volume - demarcated by the dotted circle on the 2D surface contour plots was inserted $1 \mathrm{~cm}$ from the detection surface. Scanning took place at three locations 
(top to bottom) on the gel phantoms shown by the dotted squares (probe locations) where the red circle represents the target position inside the phantom

\subsubsection{Transillumination}

Figure 52 shows the 2D surface contour plots of the processed optical signal collected employing transillumination imaging from the gel phantom. In figure 52 a region of high absorption is shown moving in the opposite direction of scanning (right to left), unlike the previously presented reflectance imaging case (see section 6.3.1) the images do contain artifacts. The expected location for the target is demarcated by a dotted circle and no metrics were calculated since the true location of the target was unknown. Objectively viewing the images as sequential scans a region of high absorption appears to be moving similarly to what was expected from the target embedded in the gel phantom. The 2D surface contour plots have various artifacts which skew the color bar. The artifacts are located on signal collected by the detectors on the outer edges of the probe. The artifacts generated are likely due to improper contact between the surface of the probe and the phantom. Transillumination imaging requires both a source and detector to be placed on opposite sides on the gel phantom (or breast tissue), which in a low level lighting environment is difficult and is a likely source of human error. Even with the artifacts on the detectors around the edges of the probe, a region of high absorption is seen moving in the direction opposite to scanning as would be expected from a target. The region of high absorption is due to the target being successfully imaged in a gel phantom employing transillumination imaging with the Gen-2 hand-held optical imager. 


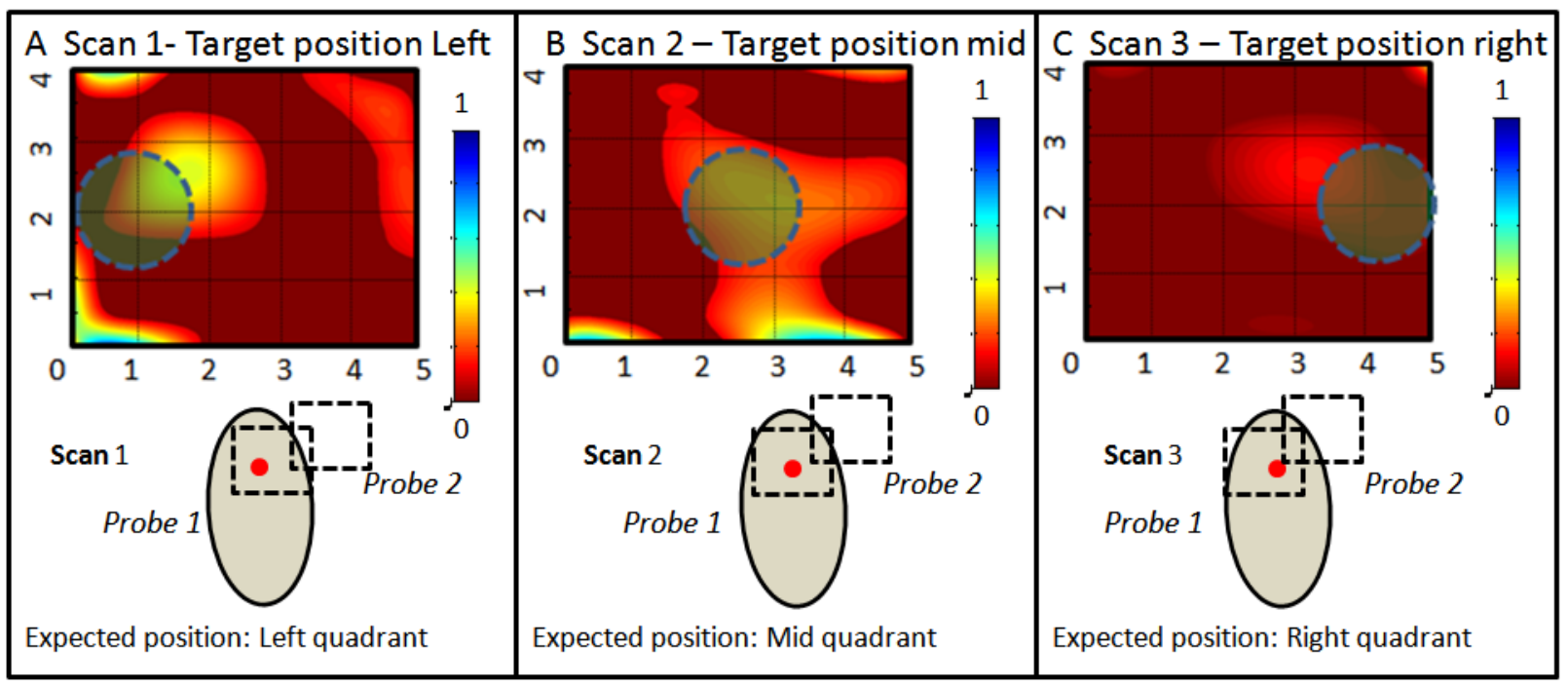

Figure 52: Transillumination imaging was performed on a gel phantom with shape and optical properties resembling human breast tissue. A $0.45 \mathrm{cc}$ India ink target $0.08 \%$ by volume - demarcated by the dotted circle on the $2 \mathrm{D}$ surface contour plots was inserted $1 \mathrm{~cm}$ from the detection surface. Scanning took place at three locations (right to left) on the gel phantoms shown by the dotted squares (the dotted squares represent the location of the two probe involved in transillumination) where the red circle represents the target position inside the phantom .

\subsection{Conclusion}

For both reflectance and transillumination imaging a region of high absorption was found moving in the direction opposite to scanning. Artifacts were either localized on the detectors near the edges (transillumination) or were not Generated (reflectance). The localization of a 0.45 cc $0.08 \%$ India ink target inside the gel phantom was likely reason for the region of high absorption in the 2D surface contour plots. This chapter provides insight into the expected problems with human breast tissue such as improper contact (alignment-source and detectors not properly aligned, pressure- differential pressure in target and background images, contact- probe not in full contact) and its effect on artifact generation. The following chapter will employ reflectance imaging on human breast tissues. 
CHAPTER 7: Reflectance imaging on human breast tissue.

\subsection{Introduction}

The focus of the chapter is to perform preliminary reflectance imaging studies on human subjects with the Gen-2 imager. In the previous chapter the Gen-2 imager was applied to breast shaped gel phantoms employing reflectance imaging suggesting that localization of a target was possible in curved tissue geometries. The Gen-2 imager (reflectance imaging) was employed to image healthy human breast tissue and subjects with cancerous breast lesions. For the healthy subjects a $0.45 \mathrm{cc}$ India ink target (contrast) employing single and dual probe imaging (bilateral imaging). The purpose of the studies is to perform preliminary in-vivo imaging of human breast tissue with Gen-2 imager.

\subsection{Materials and Methods}

\subsubsection{Instrumentation}

A Gen-2 hand-held probe has been developed with features that would overcome the limitations of Gen-1 hand-held probe (described in chapter 2 and shown in Table 4) including the ability to contour to human breast tissue geometries, homogenous illumination, and a smaller probe face. The instrumentation associated with the Gen-2 imager is found in section 3.1 where the sources, probe and detectors are discussed in detail.

\subsubsection{Experimental Studies}

A single cancer patient and three healthy human volunteers over the age of 21 were recruited (ages 20-35) in accordance with IRB protocol (IRB approval number: 071407-00 Expires May 18, 2012). The healthy volunteers were asked to place a 0.45 cc India ink target superficially under their left breast tissue (i.e. in the intra-mammary fold) at the 6 o'clock position (w.r.t. the 
reference nipple region) to simulate the endogenous absorption contrast. The subjects were also asked to position the probe over the target in accordance with the experimental studies as explained below while retaining the probe in full contact with the breast tissue.

Four experimental studies were conducted to test the feasibility of human subject imaging with the Gen-2 hand-held optical imager: (i) reflectance (single probe) imaging with healthy human subjects, (ii) effects of pressure on artifact generation and (iii) viability of simultaneous bilateral imaging on human breast tissue, and (iv) imaging of breast cancer patient with the Gen-2 handheld optical imager. CW-based reflectance imaging was performed on the breast tissue with (target) and without a target (background)

(i) Single probe reflectance imaging: An in-vivo breast imaging study was performed on 3 healthy human subjects with a $0.45 \mathrm{cc}$ absorption target ( $0.08 \%$ by volume India Ink) placed in the inter-mammary fold of their left breast. The subjects were asked to place and hold the hand-held probe (only single probe) over the breast surface right above the target (positioned in the intra-mammary fold), for collection of the (target) image. The subjects were then asked to remove the target for collection of the background image. The process was repeated for three locations (target location left middle and right of probe) using a single hand-held probe (Probe 2). The concept of multi-scanning is that, if the region(s) of high absorption were from the target location, their position would change with the scanned location on the breast tissue (as discussed in section 6.2.2 related to gel-based breast phantom studies). On the contrary, if there were no changes in the location of the high absorption regions with the movement of the probe, then these regions are potential artifacts. During the entire study, the subjects were asked to put just enough pressure on probe tissue interface to retain full contact. 
(ii) Effects of pressure on human subject imaging: The placement of the hand-held probe was found to be a source of artifact generation in the previous chapter (breast shaped gel phantoms) due to less than full contact with the geometry being imaged. Human breast tissue has less rigidity than the gel phantom previously employed. In order to visualize the effect of differential pressure between a target and background to later best determine how to mitigate or remove it a single healthy human subject with a $0.45 \mathrm{cc}$ absorption target $(0.08 \%$ by volume India Ink) placed in the infra-mammary fold of the left breast was asked to apply a incremental pressure for a series of 4 images. The pressure ranged from comfortable, slight, noticeable, and uncomfortable which were left up to the subject's interpretation.

(iii) Simultaneous bilateral imaging on a human subject: An in-vivo breast imaging study was performed with a single healthy human subject imaging study employing simultaneous bilateral reflectance imaging (see section 3.3.3). A $0.45 \mathrm{cc}$ absorption target (.8\% by volume India Ink) was placed in the intra-mammary fold of the left breast (at $6^{\prime} 0$ clock position) the contralateral right breast did not contain a target. The subject was asked to place and hold both the probes (dual probe imaging) where one probe face was placed on the left breast (target) and the other the right breast (contralateral breast -no target). A set of three images were acquired to position the target to the left, center and right of the probe (as described in the above paragraphs). The entire experiment was performed again but switching the probes, where the probe that was placed on the left breast initially was now placed on the right breast (in order to acquire the calibration image as described in section 3.3.3). The probe placement locations are shown in figure 56 below.

Placement of the target and probes were subjective and as such an accurate position for the target (true target location) was not available. However, since a scanning method was 
implemented the absorption due to the target would appear as a region of high absorption that moves in the direction opposite to scanning. Artifacts that occur due to incomplete contact, human error and uneven pressure between target and background images are likely to remain at fixed locations throughout all scans. Hence successful localization depends on producing an area of high absorption that travels in the opposite direction with respect to probe movement.

(iv) Preliminary in-vivo breast cancer imaging

Single probe reflectance imaging was employed to image a cancerous lesion (2 malignant lesions at 2 o'clock (left breast), and 1 benign lesion (L3 in US)) as shown in the x-ray and ultrasound below (Figure 53) of a 51-year old woman.
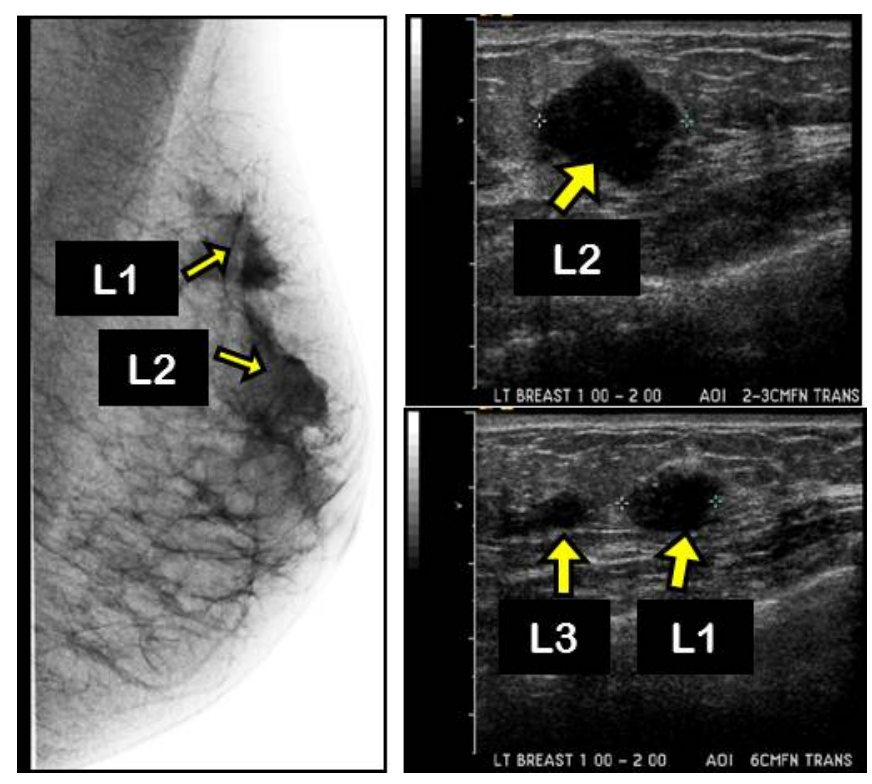

Figure 53: A single subject with multi focal cancerous legions in the left breast at approximately 2 o'clock (of the nipple region). The X-ray and ultrasound images display two large distinct legions labeled L1 and L2, while a smaller third legion is labeled L3.

Probe 2 was placed on the left breast of the subject by an experience researcher (female) to collect the target image and then was placed on the right breast to collect the background. A series of 4 locations separated by $2 \mathrm{~cm}$ in distance were imaged (locations provided in figure 57). The subject had previously attended an imaging session with the Gen-1 hand-held optical imager 
and the medical records were known, hence the approximate location of the tumor was known and the probe was placed as to image that region of the breast.

\subsubsection{Data Processing}

Experimental studies (i), (ii), (iii), and (iv) were processed as described in section 3.2. Experimental studies (i), (ii) were single probe imaging while, experimental study (iii) is simultaneous bilateral processing both are explained in chapter 3.2.5.

\subsection{Results and Discussion}

\subsection{1 (i) Single probe reflectance imaging}

2D surface contour plots of experimental study (i) are shown in figure 54. The yellow dotted circles mark the where the absorption signal due to the target is expected (region of high absorption).

For the experimental case with subject 1 there does not appear to be a region of high absorption moving in the direction opposite to scanning. There is a region of high absorption seen on the middle and right placement though not on the left. The signal observed in the $2 \mathrm{D}$ surface contour plot is likely not related to the absorption signal of the India ink target. The inability to determine if the signal observed is from the target is due to several reasons, such as

probe placement (not placed over the target), contact (insufficient contact), pressure and instrument effects.

For the experimental case with subject 2 there does appear to be a region of high absorption moving in the direction opposite to scanning. The absorption signal is faint in all regions of interest (demarcated by the yellow dotted circle), but probe placement left. In the center location there is an absorption signal though it is not shown in (blue- high absorption) due 
to the loss of contact on the sides of the probe. Observation of the $2 \mathrm{D}$ surface contour plots suggests that a region of absorption though not high absorption (likely- due to loss of contact) does move in the direction opposite to scanning.

For the experimental case with subject 3 there does appear to be a region of high absorption moving in the direction opposite to scanning. The $2 \mathrm{D}$ surface contour plots still have artifacts due to improper placement or contact though a region of high absorption is distinct and positioned within the yellow dotted rectangle where signal from the target is expected.

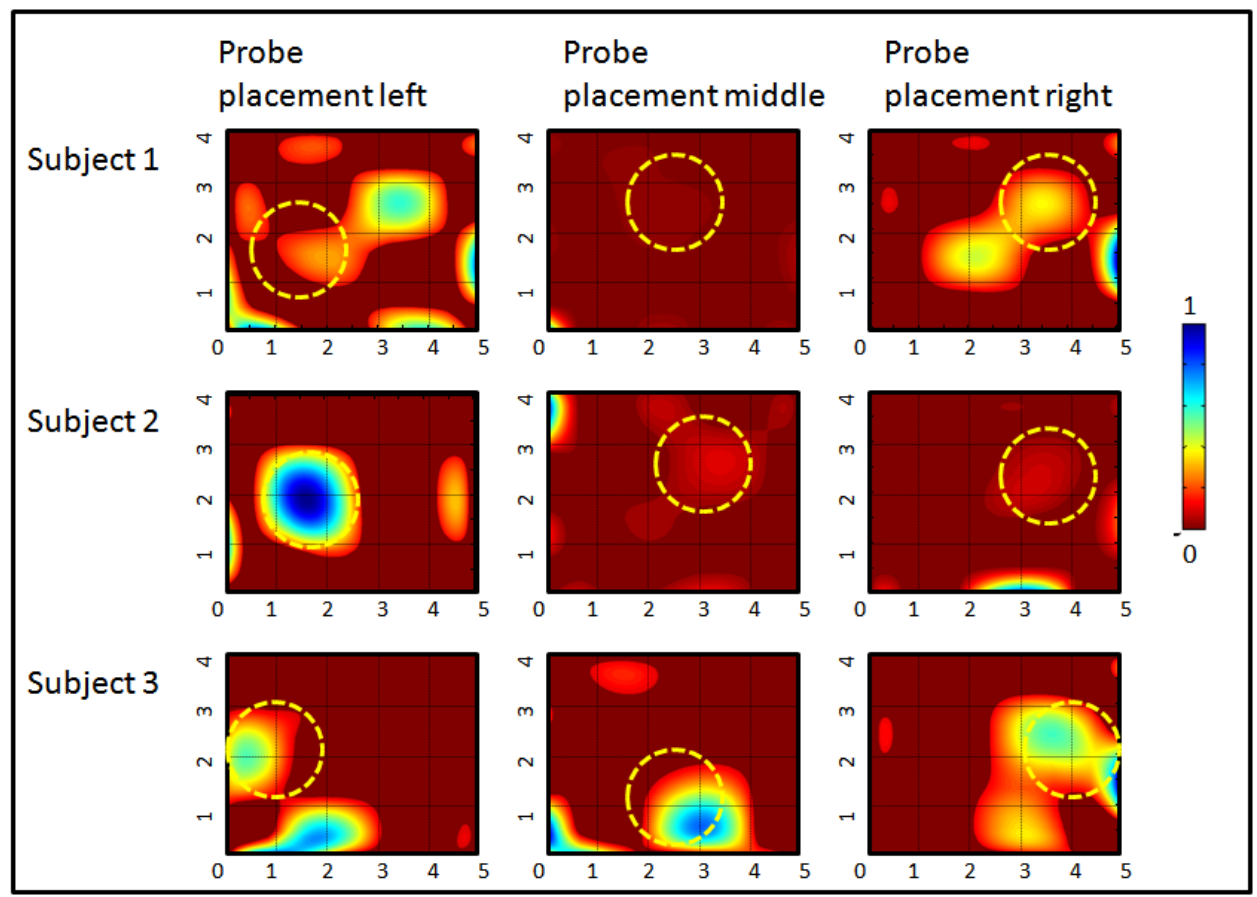

Figure 54: 2D surface contour plots of single probe reflectance imaging on breast tissue of three healthy volunteers.

The volunteers were asked to place a $0.45 \mathrm{cc}$ India ink target superficially under the flap of the left (subjects left) breast tissue (infra-mammary fold) at the 6 o'clock position (under the nipple region) to simulate the endogenous contrast of a vascular tumor. A single probe was placed on three locations by the subject as to have the target location relative to the probe on the left, middle and right regions as demarcated by the yellow circles rectangles. The target was removed for the collection of the background image to enhance contrast. 
The Gen-2 imager employing single probe reflectance imaging of a absorption based (India ink) target placed within the infra-mammary fold of healthy human breast tissue was able to localize regions of high absorption due to the target. It is unlikely that that the region of absorption found through the 2D surface contour plots (three scans left, mid and right) of subject 2 and 3 were due to artifacts.

\subsection{2 (ii) Effects of pressure on human subject imaging.}

Imaging of human breast tissue revealed a strong correlation between contact pressure and artifact generation. The effect is due to compression of the internal vasculature of the breast resulting in potential accumulation or drainage of blood between target and background images. Differential pressure between target and background image were investigated in figure 55, which shows the 2D surface contour plots of the subtracted data as processed in section 3.2.

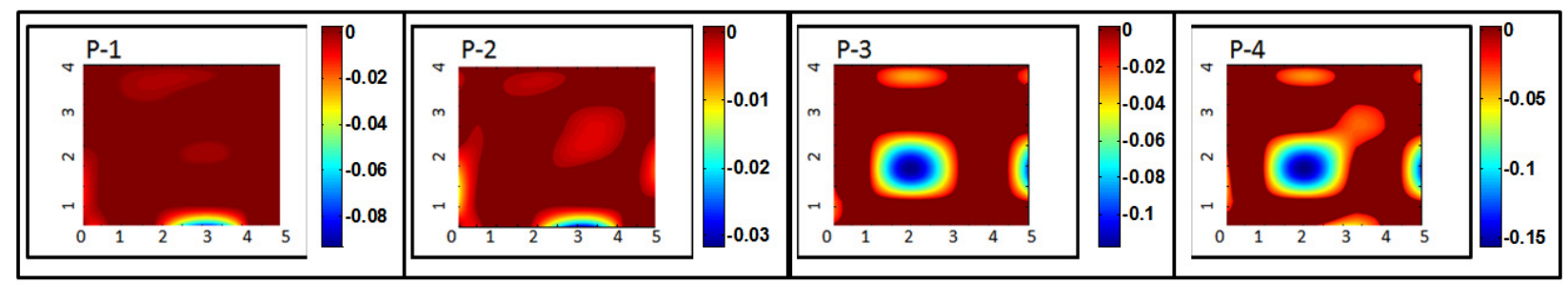

Figure 55: 2D surface contour plots of the effects of pressure on artifact generation. A single healthy human subject with a $0.45 \mathrm{cc}$ absorption target (.08\% by volume India Ink) placed in the infra-mammary fold of the left breast was asked to apply an incremental pressure for a series of 4 images. The pressure ranged from comfortable (P1), slight (P-2), noticeable (P-3), and uncomfortable (P-4) which were left up to the patient's interpretation.

The progression from P-1 to P-4 shows an increase in the difference (increase in absorption) between the target and background image (values of the color bar). An increase in absorption suggests that less light is returning to the surface, which is likely to be due to the pressure occluding the vasculature of the breast causing accumulation of blood in the tissue. If the 
absorption seen on P-3 and P-4 would have been due to a target it should have been visible in P1 and P-2 which had placement pressure similar to the background. Future modifications to the Gen-2 hand-held optical imager may include a method to monitor pressure and surface contact between the probe and the breast tissue to ensure a reduction in the Generation of artifacts during imaging.

\subsection{3 (iii) Simultaneous bilateral imaging on a human subject}

The viability of simultaneous bilateral imaging employing the Gen-2 hand-held optical imager was previously shown on liquid phantoms (see section 3.3.3), while the viability of reflectance imaging (single probe) on human subjects was first shown in section 7.3.1. 2D surface contour plots of the images collected at the three probe positions with the Gen-2 imager on a healthy human subject employing simultaneous bilateral reflectance imaging (0.45 cc India Ink target $0.8 \%$ by volume) are shown in figure 56 .

As discussed in section 3.3.3, the non-calibrated subtracted 2D surface contour plot (Step 3 in section 3.3.3) does not provide useful information due to the variability between the two probes. The non-calibrated image is a combination of the variability between the imaged tissue and the probes (the probes on the Gen-2 imager are not identical). The calibration factor (column 2 in figure) was acquired by imaging the same location with the probes resulting in an image with only the differences between the probes (Step 4 in section 3.3.3). The calibration factor between the two probes for the particular probe position was employed to adjust the non-calibrated subtracted image to contain only the variation within the imaged tissue (Step 5 in section 3.3.3). The calibrated subtracted 2D surface contour plots are shown in column 3 and 4 of figure 56 The 2D surface contour plot of calibrated subtracted data (polynomial fitted with detectors removed) for probe position 1 shows minor artifacts (Column 4, Row 1). The artifacts are likely 
due to placement errors since only absorption is located on the outer edges of the probe (poor contact between target and background images). The absorption due to target was not localized likely because the probe was not placed directly over the target.

The 2D surface contour plot of calibrated subtracted data (polynomial fitted) for probe position 2 has a region of high absorption (yellow dotted circle) on the right hand side (Column 4, Row 2). The probe had been moved in from a left position to a mid position on the breast and the $2 \mathrm{D}$ surface contour plot of calibrated subtracted data (polynomial fitted) for probe position 2 (Column 4 , Row 2) shows an absorption where probe position 1 (Column 4, Row 1) did not. The 2D surface contour plot of calibrated subtracted data (polynomial fitted) for probe position 3 shows a region of high absorption (yellow dotted circle) in the center of the probe (Column 4 , Row 3). The probe had been moved in from a mid position to a right position on the breast. The 2D surface contour plot of calibrated subtracted data (polynomial fitted) for probe position 3 (Column 4, Row 3) shows an absorption in the center of the 2D surface plot where probe position 2 (Column 4, Row 2) shows an absorption on the right of the 2D surface contour plot. An area of high absorption has been shown moving in the direction opposite to scanning as would be expected by a target. Artifacts remained constant on the outer edges of the $2 \mathrm{D}$ surface contour plots and did not move in the direction of scanning. 


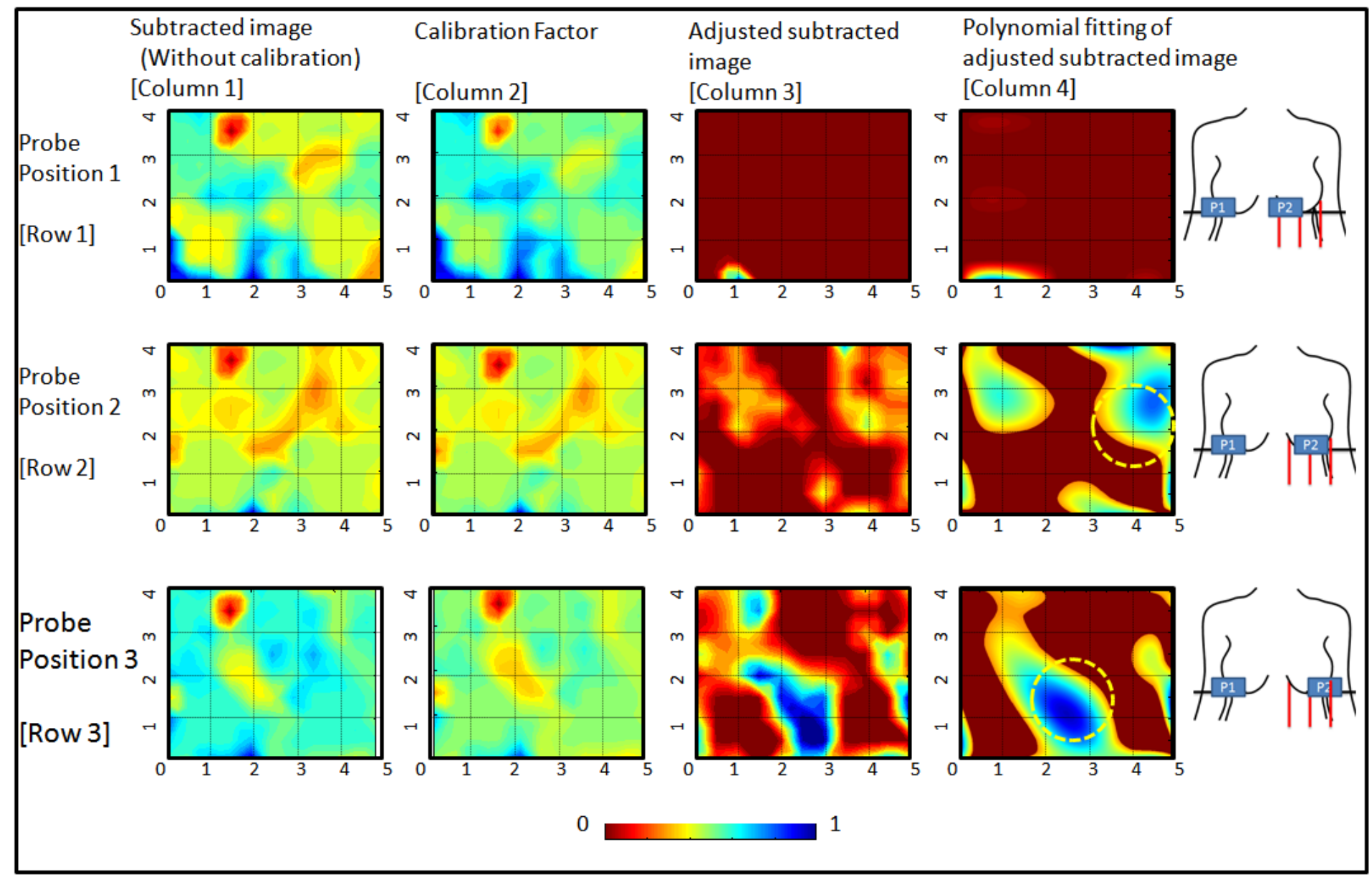

Figure 56: 2D surface contour plot of intensity distribution of progressive scans across human breast tissue with a $0.45 \mathrm{cc}$ absorption target embedded under left breast flap at 6'o clock position. Provided is the subtracted image prior to calibration, the calibration factor between the two probes , the adjusted subtracted image and the polynomial fitting of the adjusted subtracted intensity data. 


\subsection{4 (iv) Preliminary in-vivo breast cancer imaging}

The Gen-2 hand-held optical imaging system had previously been applied to liquid phantoms, gel phantoms and healthy human subjects employing a spherical $0.45 \mathrm{cc}$ India ink target to simulate the endogenous absorption contrast. A single breast cancer patient with a two distinct malignant lesions (approximately 2'oclock position from the nipple region) was imaged with the Gen-2 hand-held optical imager, where the endogenous absorption was provided by the tumor vasculature.

2D surface contour plots of the subtracted optical signal and the position of the hand-held probe during the collection of optical signal are shown in figure 57. It can be observed that the position of the probe travels (top to bottom) from over nipple region to below through the 4 progressive scans. On the 2D surface contour plots a region of high absorption travels up on each position. The movement of the hand-held probe and the optical absorption signal are in opposite directions. It is likely that the absorption signal imaged and shown in the $2 \mathrm{D}$ surface contour plots was from the endogenous contrast from the vasculature of a malignant tumors. From Figure 57 it can be seen that the tumors L1 was located approximately 10:30 o'clock from L2 (position 4 -Figure 57 and the X-ray- Figure 53). It is likely that the absorption signal shown in the $2 \mathrm{D}$ surface contour plots was from the endogenous contrast from the vasculature of malignant tumors L1 and L2. 


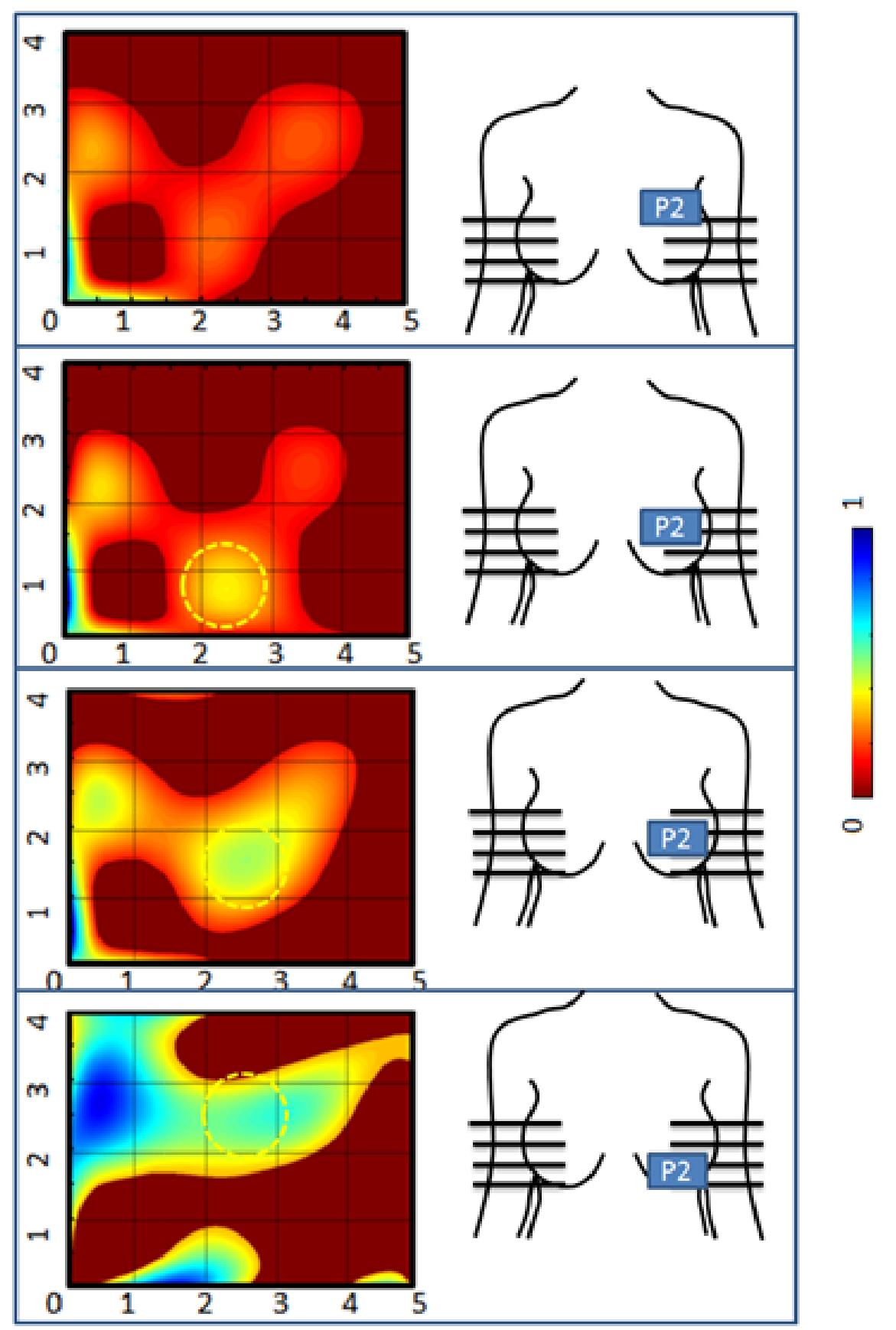

Figure 57: The Gen-2 hand-held optical imager was employed (single probe reflectance imaging) to image a cancerous legion. The X-ray and ultrasound images display two large distinct legions labeled L1 and L2, while a smaller third benign legion is labeled L3 (Figure 53) in a 51 year old woman with invasive ductal carcinoma in the left breast. The imaging method involved a sequential top to bottom scan collecting 4 images $\sim(1-2) \mathrm{cm}$ spacing (4 images collected). 


\subsection{Conclusion}

Preliminary work was performed employing the Gen-2 hand-held optical imager towards in-vivo healthy and diseased human breast tissue imaging. The work focused on performing single and dual probe reflectance imaging on healthy ( $0.45 \mathrm{cc}$ India ink target to provide endogenous contrast) and diseased human breast tissue. In all experimental cases the 2D surface contour plots of the collected optical signal showed an area of high absorption has been moving in the direction opposite to scanning as would be expected by a target. Artifacts remained constant on the outer edges of the $2 \mathrm{D}$ surface contour plots and were unlikely to move in the direction of scanning. 


\section{Conclusion}

Over the past 25 years, near-infrared (NIR) optical imaging approaches have been developed for breast cancer diagnosis based upon the endogenous absorption contrast owing to the non-specific process of angiogenesis, in order to discriminate normal from diseased tissues. In recent years, hand-held based optical imaging systems, employing the surface reflectancebased imaging configuration are developed in an attempt to translate the technology to the clinic, with maximum patient comfort and portability. A Gen-2 hand-held optical imager was developed with significant improvements over the existing Gen-1 hand-held optical imager in our laboratory. The unique features of the developed Gen-2 hand-held optical imager include: (i) a compact, portable design, similar to an ultrasound device; (ii) a fork-designed flexible probe head(s) with $\sim 86 \%$ surface contact onto different breast curvatures; (iii) A simultaneous illumination and detection setup at multiple point locations towards near real-time surface imaging of large areas; and (iv) A uniform illumination intensity distribution across multiple simultaneous point sources, allowing improvement in target detection.

Experimental studies were developed to test the improvements on the Gen-2 imager. In Chapters 4-7 experimental studies focused on the viability of the Gen-2 imager in regards to effects of homogenous illumination on target detectibility, maximum imaging depth and minimum contrast ratios where target localization could occur, image processing/scanning methods, imaging of curved contours and artifact creation with an overall goal of successfully localizing areas of high absorption in human subjects.

Optical imagers fall into two categories bed-based and hand-held where each provides different metrics due to device design. The majority of hand-held imagers provide spectroscopic information (optical properties) which depends on multiple wavelengths to differentiate healthy and un-healthy tissue [7]. The true depth information is also provided 
though it is gathered through excision of the mass or a secondary modality. The second type of optical imager, bed-based imagers are capable of 3D tomographic reconstruction which allows them to generate metrics such as target diameter, target localization and depth (optical properties with the addition of multiple wavelengths).

The Gen-2 hand-held optical imager improves the detectability of targets over the Gen-1 in terms of depth, surface contact and $\mathrm{x}, \mathrm{y}$ localization. Importantly spectroscopic hand-held optical imagers have been able to detect targets/tumors up to $\sim 4 \mathrm{~cm}$ away (spectroscopic imagers are limited to reflectance only), while the Gen-2 hand-held optical imager was capable of localizing targets at $2.5 \mathrm{~cm}$ with reflectance and $5.0 \mathrm{~cm}$ with transillumination [48-52]. A parallel plate bed-based imager developed at the University of Pennsylvania has reported the ability to detect targets through volumes of up to $6 \mathrm{~cm}$ with $3 \mathrm{D}$ tomographic reconstructions [52]. Transillumination with the Gen-2 imager has not been performed on human subjects but in phantom cases was capable of detecting absorption based targets $5 \mathrm{~cm}$ in depth without 3D tomographic reconstruction.

The hand-held optical imagers to date have not been capable transillumination or simultaneous imaging of both the breast tissues, nor single breast tissue imaging over larger areas, unlike the Gen-2 hand-held imager. Bed-based imagers have shown deeper target detection and 3D tomographic imaging but are not able to contour to tissue geometries required for the imaging of endogenous contrast between healthy and un-healthy tissues. The Gen-2 imager is a hand-held imager capable of contouring to tissue geometries, reflectance and transillumination imaging features previously only found on hand-held or bed-based imagers. The combination of features of the Gen-2 imager perform large surface area imaging while 
enhancing target detectability (including depth detection) when compared to previously developed hand-held imagers.

The Gen-2 imager to date has been a continuous wave system which generates 2D surface contour plots of only detected optical intensities. Future work for the system includes i) addition of multiple wavelengths towards the determination the optical properties or measure physiological (blood flow), ii) implementation of frequency domain equipment (which provides better image contrast than continuous wave), iii) addition of a tracking system for the hand-held probes (that allows localization of high absorption regions on the tissue surface), iv) implementation of a 3D tomographic algorithm which allows for accurate metrics of target size, and target localization, and v) implementation of fluorescence filters and equipment (sources) to employ fluorescent contrast agents towards early stage breast cancer diagnosis. 


\section{APPENDICES}

Appendix A: Simulation parameters

A forward simulation algorithm capable of simulating experiments with the Gen-2 hand-held optical imager was employed to determination of the effectiveness of a new plotting format (polynomial fitting) and determination of the maximum recovery depth for a absorption contrast agent (was used to estimate the maximum depth recovered to avoid additional testing). The simulation originally written by Godavarty et al, was modified to allow simulation of Gen-2 data [35]. The code required a $3 \mathrm{D}$ mesh of the phantom, with appropriate detector spacing corresponding to the points on the hand-held probe $(0.25 \mathrm{~cm}$ mesh spacing $)$, target diameter 0.95 $\mathrm{cm})$, the optical properties of the medium $\left(\mu_{\mathrm{a}}\right.$ and $\mu_{\mathrm{s}}$ of $.07 \mathrm{~cm}^{-1}$ and $8.5 \mathrm{~cm}^{-1}$, respectively), random noise ( $1 \%$ of maximum intensity), and contrast ratio (typically 1000:1) between the target and phantom. The forward model code was implemented as a black box to provide the output optical intensity as would be detected during experimental studies.

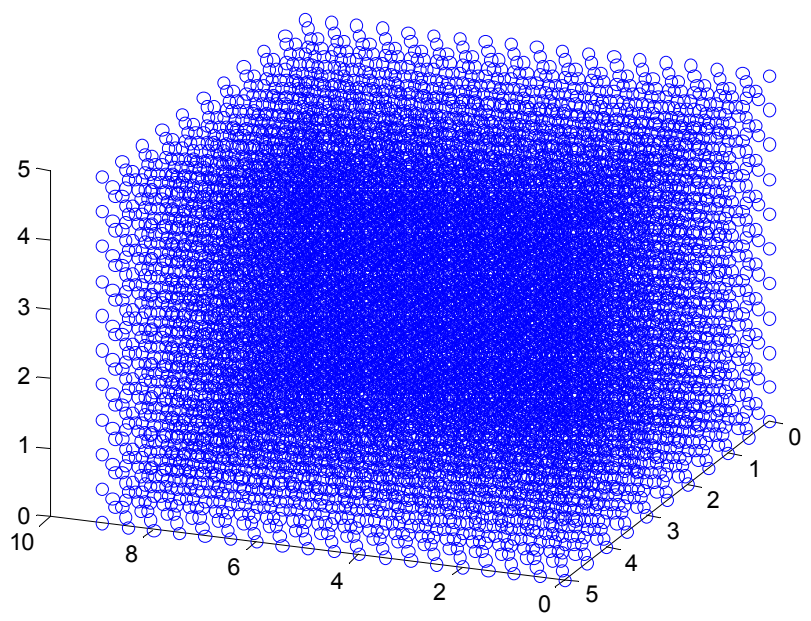

Figure A58: Mesh used during the simulation studies conducted for the Gen-2 hand-held optical imager, involving maximum recovery depth and investigation of polynomial fitting. The mesh is $5 \times 5 \times 10 \mathrm{~cm}^{3}$. 
Appendix B: Polynomial fitting

The simulation code was run using the parameters described above with a target placement of $(2,2,1) \mathrm{cm}$ from the detector surface for a single probe reflectance imaging (see section 3.3.5).

Figure B1 shows the polynomial fitting which had a recovered $(\mathrm{x}, \mathrm{y})$ location of $(1.98,2.05) \mathrm{cm}$.

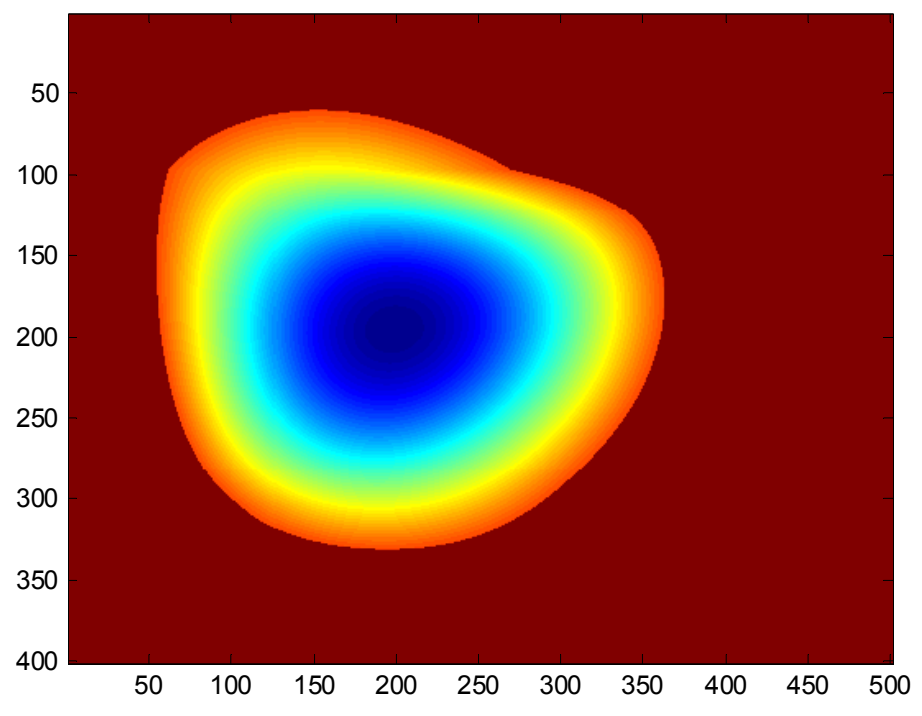

Figure B59: Simulation result of a target placed at $(2,2,1) \mathrm{cm}(\mathrm{x}, \mathrm{y}, \mathrm{z})$ from the detection surface employing single probe reflectance imaging. 

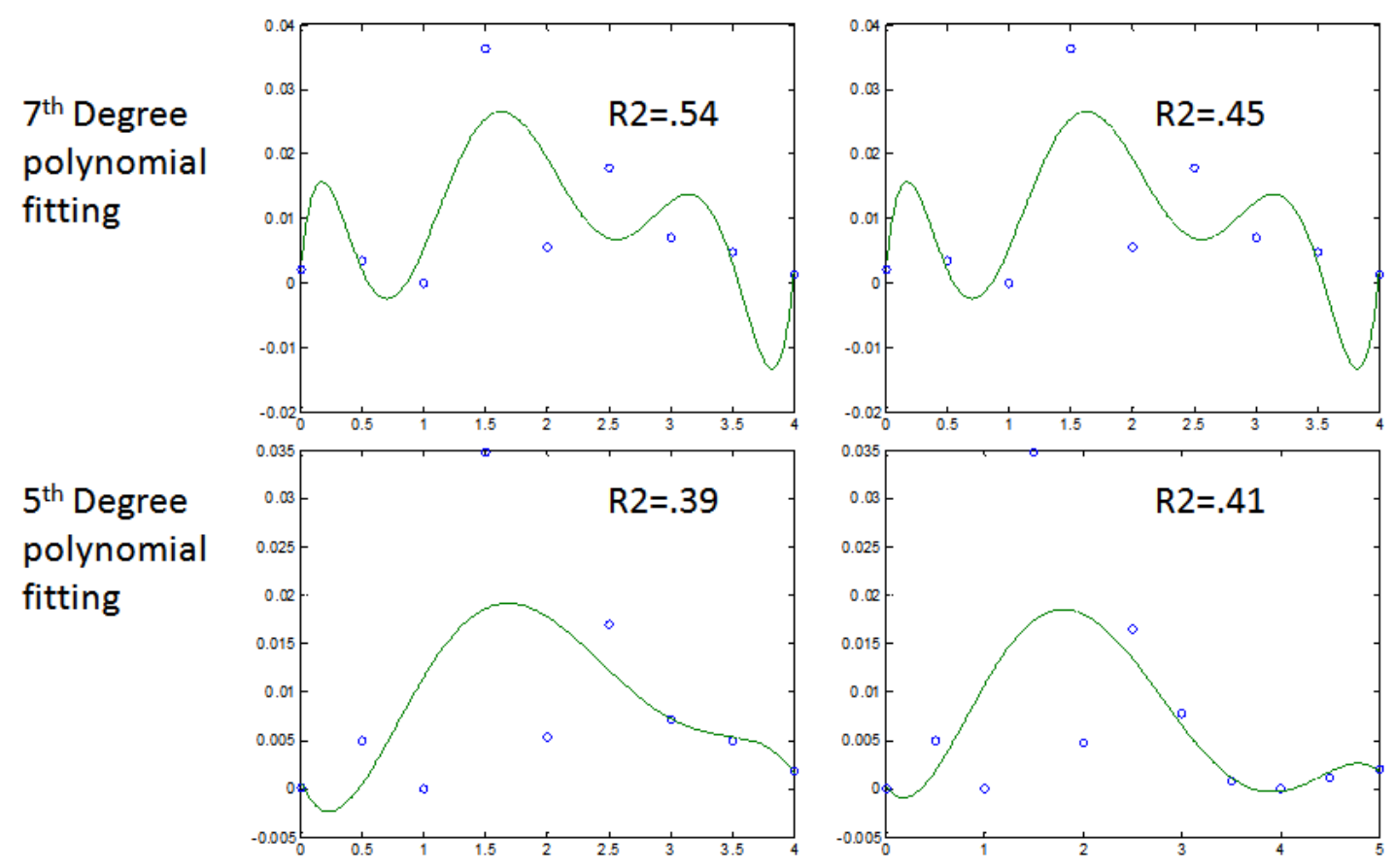

Figure B60: Comparison of polynomial fitting of 7th and 5th degree. The figure shows several peaks for the 7th degree which negate its improved $\mathrm{R}^{2}$ value.

Appendix C: Simulation depth limitation

The results displayed that the maximum depth recovered was $\sim 2.5 \mathrm{~cm}$

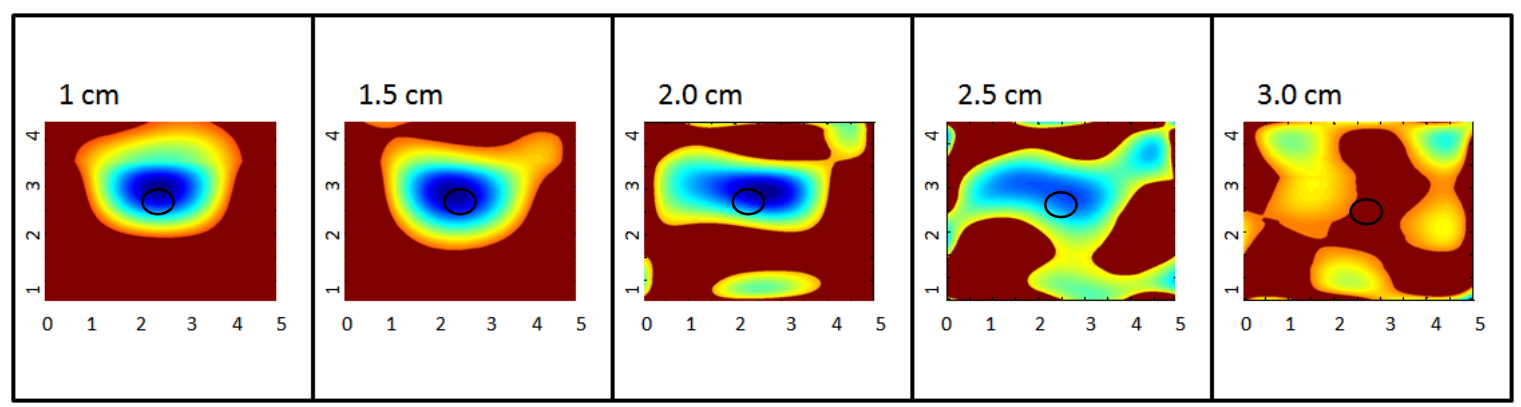

Figure C61: Simulation of a spherical $(0.95 \mathrm{~cm}$ diameter) absorption contrast target at various depths at a background contrast of 1:0. The depths ranged from $1-3.0 \mathrm{~cm}$ at a $0.5 \mathrm{~cm}$ spacing with the center of the circle at location $2.5,2.5 \mathrm{~cm}-\mathrm{x}, \mathrm{y}$ as demarcated by a black circle 
The figures were generated using $\mu_{\mathrm{a}}$ and $\mu_{\mathrm{s}}$ of $0.07 \mathrm{~cm}^{-1}$ and $8.5 \mathrm{~cm}^{-1}$, respectively using a contrast between target and background 1000:1. Artificial noise was used at 1\% of maximum intensity to better estimate an experimental case. Comparison with experimental studies performed later displayed qualitatively similarity between the experimental and simulation 2D surface contour plots. 
Appendix D: Quantitative analysis of extensive reflectance and transillumination experimental phantom studies.

The calculated values for detected target location, distance between detected location and true location, recovered target to background ratio, Estimated target diameter and measurement error for all experimental cases of transillumination and reflectance are listed below. The table's distance between true and recovered locations are the criteria between a successful and unsuccessful localization (as was presented in table 9-10 in section 5.2.2). A successful localization depends on the cutoff value of $1 \mathrm{~cm}$ and to some extent can be based on recovered target:background ratio as better localization Generates higher values. The recovered target:background ratios or contrast range from 20 to 0.5 from successful and unsuccessful cases. Measurement error ranges from $\sim .0005-.23$ and serves as a value of camera performance and not target localization. 
Extensive Reflectance:

\begin{tabular}{|c|c|c|c|c|c|c|c|c|c|}
\hline & Background & $\begin{array}{l}\text { True } \\
\text { Location }\end{array}$ & Depth & $\begin{array}{l}\text { Detected } \\
\text { location X } \\
\text { (cm) }\end{array}$ & $\begin{array}{l}\text { Detected } \\
\text { location } \\
\mathrm{Y}(\mathrm{cm})\end{array}$ & $\begin{array}{l}\text { Detected } \\
\text { location } \\
\mathrm{x}, \mathrm{y}, \mathrm{x}(\mathrm{cm})\end{array}$ & $\begin{array}{l}\text { Recovered } \\
\text { target:background } \\
\text { ratio }\end{array}$ & $\begin{array}{l}\text { Estimated } \\
\text { Target } \\
\text { diameter }\end{array}$ & $\begin{array}{l}\text { Measurement } \\
\text { error }\end{array}$ \\
\hline \multirow[t]{10}{*}{0.08} & $1: 0 \mathrm{a}$ & $\mathrm{x}, \mathrm{y}(\mathrm{cm})$ & $\begin{array}{l}\mathrm{Z} \\
(\mathrm{cm})\end{array}$ & & & & & & \\
\hline & & $(1,2.5)$ & 1 & 2.59 & 1.92 & 0.12 & 8.3 & 1.88 & 0.218 \\
\hline & & $(1,2.5)$ & 1.5 & 2.47 & 2 & 0.03 & 7.4 & 1.94 & 0.216 \\
\hline & & $(1,2.5)$ & 2 & 2.7 & 2.02 & 0.2 & 4.3 & 0.78 & 2.18 \\
\hline & & $(1,2.5)$ & 2.5 & 2.46 & 1.9 & 0.11 & 12.5 & 0.94 & 0.211 \\
\hline & 1000:1 a & $\mathrm{x}, \mathrm{y}(\mathrm{cm})$ & $\begin{array}{l}\mathrm{Z} \\
(\mathrm{cm})\end{array}$ & & & & & & \\
\hline & & $(1,2.5)$ & 1 & 2.12 & 2.15 & 0.41 & 7.8 & 1.6 & 0.212 \\
\hline & & $(1,2.5)$ & 1.5 & 2.22 & 1.95 & 0.28 & 6.4 & 1.8 & 0.216 \\
\hline & & $(1,2.5)$ & 2 & 2.61 & 1.65 & 0.37 & 5.1 & 1.8 & 0.217 \\
\hline & & $(1,2.5)$ & 2.5 & 2.6 & 1.18 & 0.83 & 2.26 & 2 & 0.219 \\
\hline
\end{tabular}




\begin{tabular}{|c|c|c|c|c|c|c|c|c|c|}
\hline & $100: 1 \mathrm{a}$ & $\mathrm{x}, \mathrm{y}(\mathrm{cm})$ & $(\mathrm{cm})$ & & & & & & \\
\hline & & $(1,2.5)$ & 1 & 2.12 & 2.16 & 0.41 & 8.8 & 1.53 & 0.211 \\
\hline & & $(1,2.5)$ & 1.5 & 2.14 & 2.15 & \begin{tabular}{|l|}
0.39 \\
\end{tabular} & 8.2 & 1.62 & 0.211 \\
\hline & & $(1,2.5)$ & 2 & 2.15 & 2.14 & \begin{tabular}{|l|l|}
0.38 \\
\end{tabular} & 9.3 & 1.54 & 0.211 \\
\hline & & $(1,2.5)$ & 2.5 & 2.27 & 2.08 & \begin{tabular}{|l|l|}
0.24 \\
\end{tabular} & 5 & 1.62 & 0.218 \\
\hline & $10: 1 \mathrm{~b}$ & $\mathrm{x}, \mathrm{y}(\mathrm{cm})$ & $\begin{array}{l}\mathrm{z} \\
(\mathrm{cm})\end{array}$ & & & & & & \\
\hline & & $(1,2.5)$ & 1 & 2.53 & 1.81 & \begin{tabular}{|l|}
0.19 \\
\end{tabular} & 9.1 & 1.8 & 0.206 \\
\hline & & $(1,2.5)$ & 1.5 & 2.44 & 1.77 & 0.24 & 6.4 & 1.07 & 0.213 \\
\hline & & $(1,2.5)$ & 2 & 5.49 & 0.42 & 3.4 & 3.9 & 0.64 & 0.215 \\
\hline & & $(1,2.5)$ & 2.5 & 0 & 0.5 & 2.9 & 1.7 & 0.48 & 0.214 \\
\hline \multirow[t]{3}{*}{0.008} & $1: 0 \mathrm{a}$ & $\mathrm{x}, \mathrm{y}(\mathrm{cm})$ & $\begin{array}{l}\mathrm{z} \\
(\mathrm{cm})\end{array}$ & & & & & & \\
\hline & & $(1,2.5)$ & 1 & 2.64 & 1.88 & \begin{tabular}{|l|l|}
0.18 \\
\end{tabular} & 6.7 & 1.25 & 0.217 \\
\hline & & \begin{tabular}{|l}
$(1,2.5)$ \\
\end{tabular} & 1.5 & 2.76 & 1.83 & \begin{tabular}{|l|}
0.31 \\
\end{tabular} & 7.6 & 1.10 & 0.224 \\
\hline
\end{tabular}




\begin{tabular}{|c|c|c|c|c|c|c|c|c|}
\hline & $(1,2.5)$ & 2 & 2.74 & 2.33 & 0.41 & 3.5 & 1.38 & 0.220 \\
\hline & $(1,2.5)$ & 2.5 & 2.44 & 0.74 & 1.3 & 1.5 & 1.4 & 0.220 \\
\hline $1000: 1 \mathrm{a}$ & $\mathrm{x}, \mathrm{y}(\mathrm{cm})$ & $\begin{array}{l}\mathrm{Z} \\
(\mathrm{cm})\end{array}$ & & & & & & \\
\hline & $(1,2.5)$ & 1 & 2.29 & 2.11 & 0.24 & 7.9 & 0.95 & 0.196 \\
\hline & $(1,2.5)$ & 1.5 & 2.39 & 1.82 & 0.21 & 3.9 & 1.1 & 0.205 \\
\hline & $(1,2.5)$ & 2 & 2.54 & 1.74 & 0.26 & 2.9 & 0.84 & 0.197 \\
\hline & $(1,2.5)$ & 2.5 & 2.42 & 3.29 & 1.3 & 5.8 & 1.2 & 0.192 \\
\hline $100: 1 \mathrm{a}$ & $\mathrm{x}, \mathrm{y}(\mathrm{cm})$ & $\begin{array}{l}\mathrm{Z} \\
(\mathrm{cm})\end{array}$ & & & & & & \\
\hline & $(1,2.5)$ & 1 & 2.15 & 2.22 & 0.41 & 7.6 & 0.62 & 0.211 \\
\hline & $(1,2.5)$ & 1.5 & 2.36 & 2.27 & 0.30 & 8.6 & 0.60 & 0.214 \\
\hline & $(1,2.5)$ & 2 & 2.52 & 2.09 & 0.09 & 9.3 & 1.4 & 0.219 \\
\hline & $(1,2.5)$ & 2.5 & 3.5 & 2.11 & 1.00 & 2.9 & 0.39 & 0.222 \\
\hline $10: 1 \mathrm{~b}$ & $\mathrm{x}, \mathrm{y}(\mathrm{cm})$ & $\begin{array}{l}\mathrm{Z} \\
(\mathrm{cm})\end{array}$ & & & & & & \\
\hline
\end{tabular}




\begin{tabular}{|c|c|c|c|c|c|c|c|c|c|}
\hline & & $(1,2.5)$ & 1 & 2.48 & 2.14 & 0.14 & 8.3 & 0.67 & 0.205 \\
\hline & & $(1,2.5)$ & 1.5 & 2.42 & 2.26 & 0.27 & 9.3 & 0.65 & 0.210 \\
\hline & & $(1,2.5)$ & 2 & 3.04 & 1 & 1.14 & 0.21 & 0 & 0.211 \\
\hline & & $(1,2.5)$ & 2.5 & 0 & 0.82 & 2.8 & 2.0 & 0.86 & 0.214 \\
\hline \multirow[t]{10}{*}{0.8} & $1: 0 \mathrm{a}$ & $\mathrm{x}, \mathrm{y}(\mathrm{cm})$ & $\begin{array}{l}\mathrm{Z} \\
(\mathrm{cm})\end{array}$ & & & & & & \\
\hline & & $(2.5,2.5)$ & 1 & 2.66 & 2.43 & 0.17 & 7.6 & 0.43 & 0.22 \\
\hline & & $(2.5,2.5)$ & 1.5 & 2.43 & 2.38 & 0.14 & 6.9 & 0.48 & 0.234 \\
\hline & & $(2.5,2.5)$ & 2 & 2.39 & 2.36 & 0.18 & 5.8 & 1.7 & 0.240 \\
\hline & & $(2.5,2.5)$ & 2.5 & 2.07 & 2.43 & 0.44 & 5.2 & 0.92 & 0.242 \\
\hline & $1000: 1 \mathrm{a}$ & $\mathrm{x}, \mathrm{y}(\mathrm{cm})$ & $\begin{array}{l}\mathrm{Z} \\
(\mathrm{cm})\end{array}$ & & & & & & \\
\hline & & $(2.5,2.5)$ & 1 & 2.58 & 2.16 & 0.18 & 5.5 & 0.90 & 0.188 \\
\hline & & $(2.5,2.5)$ & 1.5 & 2.48 & 2.3 & 0.30 & 4.5 & 0.93 & 0.206 \\
\hline & & $(2.5,2.5)$ & 2 & 2.67 & 2.71 & 0.73 & 5.4 & 1.2 & 0.205 \\
\hline & & $(2.5,2.5)$ & 2.5 & 2.48 & 3.37 & 1.4 & 3.9 & 1.1 & 0.216 \\
\hline
\end{tabular}




\begin{tabular}{|c|c|c|c|c|c|c|c|c|}
\hline $100: 1 \mathrm{a}$ & $\mathrm{x}, \mathrm{y}(\mathrm{cm})$ & $\begin{array}{l}\mathrm{Z} \\
(\mathrm{cm})\end{array}$ & & & & & & \\
\hline & $(2.5,2.5)$ & 1 & 2.65 & 1.65 & 0.38 & 10. & 1.3 & 0.210 \\
\hline & $(2.5,2.5)$ & 1.5 & 2.85 & 1.58 & 0.55 & 12. & 0.98 & 0.220 \\
\hline & $(2.5,2.5)$ & 2 & 2.85 & 1.61 & 0.52 & 10. & 1.1 & 0.215 \\
\hline & $(2.5,2.5)$ & 2.5 & 4.36 & 0.01 & 2.7 & 3.2 & 0.45 & 0.212 \\
\hline $10: 1 \mathrm{~b}$ & $\mathrm{x}, \mathrm{y}(\mathrm{cm})$ & $\begin{array}{l}\mathrm{Z} \\
(\mathrm{cm})\end{array}$ & & & & & & \\
\hline & $(2.5,2.5)$ & 1 & 4.5 & 3.99 & 2.5 & 3.7 & 0.64 & 0.162 \\
\hline & $(2.5,2.5)$ & 1.5 & 4.51 & 3.99 & 2.50 & 0 & 4.1 & 0.160 \\
\hline & $(2.5,2.5)$ & 2 & 3.64 & 2.53 & 1.1 & 3.8 & 0.58 & 0.16 \\
\hline & $(2.5,2.5)$ & 2.5 & 4.99 & 1.93 & 2.6 & 0 & 4.8 & 0.164 \\
\hline
\end{tabular}

Table 14: The calculated, true target location, target size, contrast, and measurement error for all experimental cases of reflectance performed. 
Extensive Transillumination:

\begin{tabular}{|c|c|c|c|c|c|c|c|c|c|}
\hline & Background & $\begin{array}{l}\text { True } \\
\text { Location }\end{array}$ & Depth & $\begin{array}{l}\text { Detected } \\
\text { Location } \\
\mathrm{X}\end{array}$ & $\begin{array}{l}\text { Detected } \\
\text { location } \\
\mathrm{Y}\end{array}$ & $\begin{array}{l}\text { Detected } \\
\text { location } \\
\mathrm{x}, \mathrm{y},(\mathrm{cm})\end{array}$ & $\begin{array}{l}\text { Recovered } \\
\text { target:background } \\
\text { ratio }\end{array}$ & $\begin{array}{l}\text { Estimated } \\
\text { Target diameter }\end{array}$ & $\begin{array}{l}\text { Measurement } \\
\text { error }\end{array}$ \\
\hline \multirow[t]{10}{*}{0.08} & $1: 0 \mathrm{a}$ & $\mathrm{x}, \mathrm{y}(\mathrm{cm})$ & $\begin{array}{l}\mathrm{Z} \\
(\mathrm{cm})\end{array}$ & & & & & & \\
\hline & & $(1.5,2.0)$ & 1 & 1.65 & 2.15 & 0.21 & 19.5 & 1.35 & 0.0008 \\
\hline & & $(1.5,2.0)$ & 2 & 1.65 & 2.3 & 0.33 & 18.1 & 2 & 0.0005 \\
\hline & & $(1.5,2.0)$ & 3 & 1.57 & 2.28 & 0.288 & 15.7 & 1.2 & 0.0006 \\
\hline & & $(1.5,2.0)$ & 4 & 1.53 & 2.1 & 0.1 & 22.1 & 0.19 & 0.0007 \\
\hline & & $(1.5,2.0)$ & 5 & 1.6 & 1.92 & 0.13 & 15.1 & 0.19 & 0.001 \\
\hline & $1000: 1 \mathrm{a}$ & $\mathrm{x}, \mathrm{y}(\mathrm{cm})$ & $\begin{array}{l}\mathrm{Z} \\
(\mathrm{cm})\end{array}$ & & & & & & \\
\hline & & $(1,2.5)$ & 1 & 0.93 & 2.47 & 0.1 & 5.9 & 2.8 & 0.200 \\
\hline & & $(1,2.5)$ & 2 & 0.87 & 2.5 & 0.13 & 6.1 & 2.6 & 0.204 \\
\hline & & $(1,2.5)$ & 3 & 1.04 & 2.65 & 0.16 & 5 & 3.3 & 0.196 \\
\hline
\end{tabular}




\begin{tabular}{|c|c|c|c|c|c|c|c|c|}
\hline & $(1,2.5)$ & 4 & 0.9 & 2.71 & 0.23 & 5.6 & 0.65 & 0.199 \\
\hline & & 5 & 0.75 & 2.93 & 0.5 & 5 & 2.7 & 0.194 \\
\hline $100: 1 \mathrm{a}$ & $\mathrm{x}, \mathrm{y}(\mathrm{cm})$ & $\begin{array}{l}\mathrm{Z} \\
(\mathrm{cm})\end{array}$ & & & & & & \\
\hline & $(1,2.5)$ & 1 & 0.55 & 2.82 & 0.55 & 3.8 & 1.24 & 0.252 \\
\hline & $(1,2.5)$ & 2 & 0.63 & 2.79 & 0.47 & 4.4 & 1.14 & 0.254 \\
\hline & $(1,2.5)$ & 3 & 0.61 & 2.82 & 0.5 & 3.9 & 0.95 & 0.241 \\
\hline & $(1,2.5)$ & 4 & 0.6 & 2.85 & 0.53 & 4.3 & 0.97 & 0.248 \\
\hline & & 5 & 0.57 & 2.74 & 0.49 & 5.8 & 0.74 & 0.241 \\
\hline $10: 1 \mathrm{~b}$ & $\mathrm{x}, \mathrm{y}(\mathrm{cm})$ & $\begin{array}{l}\mathrm{Z} \\
(\mathrm{cm})\end{array}$ & & & & & & \\
\hline & $(1,2.5)$ & 1 & 1.78 & 0 & 2.6 & 5.01 & 1.9 & 0.211 \\
\hline & $(1,2.5)$ & 2 & 2.16 & 1.19 & 1.7 & 5 & 0.96 & 0.238 \\
\hline & $(1,2.5)$ & 3 & 1.4 & 3.88 & 1.4 & 5 & 1.3 & 0.217 \\
\hline & $(1,2.5)$ & 4 & 2.27 & 0 & 2.8 & 5 & 0.69 & 0.232 \\
\hline & & 5 & 1.62 & 2.46 & 0.62 & 4.9 & 0.89 & 0.216 \\
\hline
\end{tabular}




\begin{tabular}{|c|c|c|c|c|c|c|c|c|c|}
\hline 0.008 & $1: 0 \mathrm{a}$ & $\mathrm{x}, \mathrm{y}(\mathrm{cm})$ & $\begin{array}{l}\mathrm{Z} \\
(\mathrm{cm})\end{array}$ & & & & & & \\
\hline & & $(2.5,2.0)$ & 1 & 2.51 & 1.95 & 0.05 & 8.4 & 0.01 & 0.230 \\
\hline & & $(2.5,2.0)$ & 2 & 2.41 & 1.9 & 0.13 & 6.1 & 1.7 & 0.223 \\
\hline & & $(2.5,2.0)$ & 3 & 2.58 & 1.68 & 0.33 & 4.8 & 1.1 & 0.249 \\
\hline & & $(2.5,2.0)$ & 4 & 2.45 & 2.14 & 0.15 & 5.9 & 0.90 & 0.209 \\
\hline & & $(2.5,2.0)$ & 5 & 2.25 & 1.76 & 0.20 & 7.9 & 0.39 & 0.215 \\
\hline $\mathrm{c}$ & $1000: 1 \mathrm{a}$ & $\mathrm{x}, \mathrm{y}(\mathrm{cm})$ & $\begin{array}{l}\mathrm{Z} \\
(\mathrm{cm})\end{array}$ & & & & & & \\
\hline & & $(1.5,2.0)$ & 1 & 1.56 & 1.7 & 0.31 & 4.7 & 1.0 & 0.197 \\
\hline & & $(1.5,2.0)$ & 2 & 1.66 & 1.94 & 0.17 & 2.9 & 0.89 & 0.215 \\
\hline & & $(1.5,2.0)$ & 3 & 1.76 & 1.55 & 0.52 & 5.3 & 0.84 & 0.204 \\
\hline & & $(1.5,2.0)$ & 4 & 1.84 & 1.39 & 0.69 & 5.04 & 0.90 & 0.208 \\
\hline & & $(1.5,2.0)$ & 5 & 1.96 & 1.97 & 0.46 & 3.9 & 1.0 & 0.206 \\
\hline c & $100: 1 \mathrm{a}$ & $\mathrm{x}, \mathrm{y}(\mathrm{cm})$ & $\begin{array}{l}\mathrm{Z} \\
(\mathrm{cm})\end{array}$ & & & & & & \\
\hline
\end{tabular}




\begin{tabular}{|c|c|c|c|c|c|c|c|c|c|}
\hline & & $(1,2.5)$ & 1 & 1.68 & 3.86 & 1.5 & 1.1 & 0.7 & 0.228 \\
\hline & & $(1,2.5)$ & 2 & 4.08 & 2.31 & 3.1 & 5.2 & 0.59 & 0.209 \\
\hline & & $(1,2.5)$ & 3 & 3.05 & 3.45 & 2.3 & 4.9 & 0.75 & 0.215 \\
\hline & & $(1,2.5)$ & 4 & 4.41 & 3.1 & 3.5 & 3.9 & 0.69 & 0.221 \\
\hline & & & 5 & 4.68 & 3.83 & 3.9 & 5.2 & 1.5 & 0.217 \\
\hline \multirow[t]{6}{*}{ c } & $10: 1 \mathrm{~b}$ & $\mathrm{x}, \mathrm{y}(\mathrm{cm})$ & $\begin{array}{l}\mathrm{Z} \\
(\mathrm{cm})\end{array}$ & & & & & & \\
\hline & & $(1,2.5)$ & 1 & 2.14 & 1.8 & 1.3 & 5.4 & 0.79 & 0.215 \\
\hline & & $(1,2.5)$ & 2 & 3.65 & 2.75 & 2.7 & 2.2 & 1.1 & 0.223 \\
\hline & & $(1,2.5)$ & 3 & 0.26 & 1.57 & 1.56 & 4.5 & 1.6 & 0.226 \\
\hline & & $(1,2.5)$ & 4 & 4.21 & 2.88 & 3.2 & 3.9 & 1.2 & 0.217 \\
\hline & & $(1,2.5)$ & 5 & 2.74 & 2.44 & 1.7 & 2.7 & 2.3 & 0.228 \\
\hline \multirow[t]{3}{*}{0.8} & $1: 0 a$ & $\mathrm{x}, \mathrm{y}(\mathrm{cm})$ & $\begin{array}{l}\mathrm{Z} \\
(\mathrm{cm})\end{array}$ & & & & & & \\
\hline & & $(2.5,2.0)$ & 1 & 2.35 & 1.89 & 0.19 & 6.5 & 1.8 & 0.005 \\
\hline & & $(2.5,2.0)$ & 2 & 2.17 & 2.17 & 0.37 & 7.1 & 1.7 & 0.002 \\
\hline
\end{tabular}




\begin{tabular}{|c|c|c|c|c|c|c|c|c|c|}
\hline & & $(2.5,2.0)$ & 3 & 2.19 & 1.88 & 0.33 & 7.7 & 1.4 & 0.002 \\
\hline & & $(2.5,2.0)$ & 4 & 2.15 & 1.88 & 0.37 & 6.9 & 1.3 & 0.002 \\
\hline & & $(2.5,2.0)$ & 5 & 2.09 & 1.73 & 0.49 & 6.7 & 1.1 & 0.003 \\
\hline & $1000: 1 \mathrm{a}$ & $\mathrm{x}, \mathrm{y}(\mathrm{cm})$ & $\begin{array}{l}\mathrm{Z} \\
(\mathrm{cm})\end{array}$ & & & & & & \\
\hline & & $(1.0,2.5)$ & 1 & 0.55 & 2.51 & 0.45 & 4.9 & 1.4 & 0.225 \\
\hline & & $(1.0,2.5)$ & 2 & 0.54 & 2.56 & 0.46 & 4.8 & 0.50 & 0.191 \\
\hline & & $(1.0,2.5)$ & 3 & 0.53 & 2.46 & 0.47 & 4.9 & 1.4 & 0.196 \\
\hline & & $(1.0,2.5)$ & 4 & 0.5 & 2.57 & 0.50 & 5.1 & 1.1 & 0.203 \\
\hline & & $(1.0,2.5)$ & 5 & 0.48 & 2.62 & 0.53 & 5.3 & 0.83 & 0.189 \\
\hline $\mathrm{c}$ & $100: 1 \mathrm{a}$ & $\mathrm{x}, \mathrm{y}(\mathrm{cm})$ & $\begin{array}{l}\mathrm{Z} \\
(\mathrm{cm})\end{array}$ & & & & & & \\
\hline & & $(1,2.5)$ & 1 & 3.92 & 2.93 & 2.9 & 4.9 & 1.8 & 0.203 \\
\hline & & $(1,2.5)$ & 2 & 4 & 3 & 3.0 & 5.4 & 0.33 & 0.224 \\
\hline & & $(1,2.5)$ & 3 & 4.08 & 3.02 & .59 & 4.5 & 0.53 & 0.207 \\
\hline & & $(1,2.5)$ & 4 & 4 & 3.04 & .89 & 5.4 & 0.78 & 0.220 \\
\hline
\end{tabular}




\begin{tabular}{|c|c|c|c|c|c|c|c|c|c|}
\hline & & $(1,2.5)$ & 5 & 4 & 2.96 & 1.03 & 5.1 & 1.3 & 0.226 \\
\hline $\mathrm{c}$ & $10: 1 \mathrm{~b}$ & $\mathrm{x}, \mathrm{y}(\mathrm{cm})$ & $\begin{array}{l}\mathrm{Z} \\
(\mathrm{cm})\end{array}$ & & & & & & \\
\hline & & $(1,2.5)$ & 1 & 5 & 3.92 & 4.3 & 5.1 & 0.48 & 0.230 \\
\hline & & $(1,2.5)$ & 2 & 3.35 & 3 & 2.4 & 5.3 & 1.9 & 0.228 \\
\hline & & $(1,2.5)$ & 3 & 4.26 & 2.95 & 3.3 & 5.2 & 0.29 & 0.220 \\
\hline & & $(1,2.5)$ & 4 & 3.06 & 3.91 & 2.5 & 5.0 & 0.98 & 0.219 \\
\hline & & $(1,2.5)$ & 5 & 2.68 & 3.59 & 2.0 & 4.9 & 0.56 & 0.195 \\
\hline
\end{tabular}

Table 15: The calculated, true target location, target size, contrast, and measurement error for all experimental cases of transillumination performed 
Appendix E: Measurement of optical properties

To determine the optical properties of the solid phantoms a frequency domain based spectroscopy system was employed. The system applies homodyne detection for the acquisition of the frequency domain data. Figure 62 demonstrates the direction of signal from source to detector.

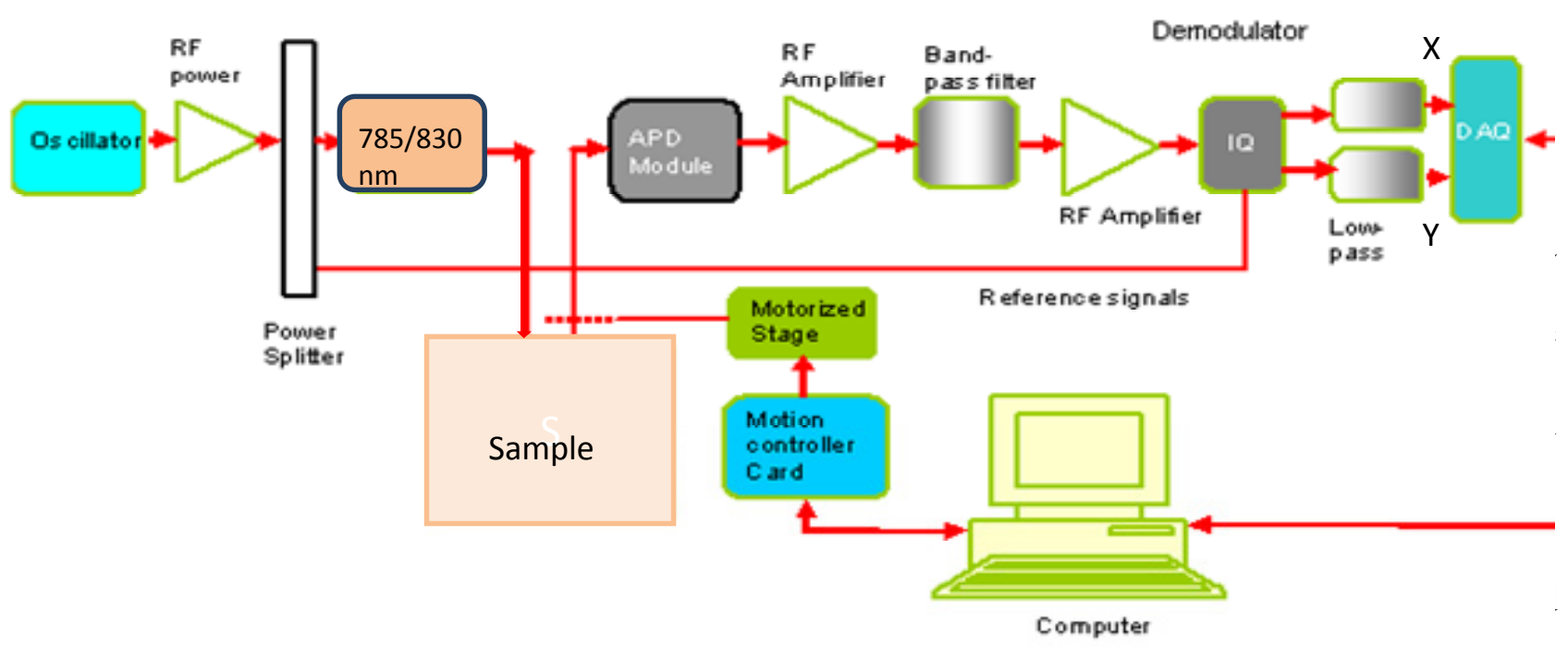

as the reference signal. The other portion is sent into the phantom where the light is scattered and absorbed. A second optical fiber collects the signal and relays it to an avalanche photo diode (APD) (Hamamatsu C5331-30) where a conversion from optical to electrical signal occurs (voltage). The signal is then undergoes through phases of amplification and demodulation. Demodulated in-phase (I) and quadrature (Q) voltages are further filtered by a low-pass filter, which are then sampled by a data acquisition (DAQ) board (National Instruments PCI-6281). From the two sampled components, the amplitude and phase signals can be obtained for a specific source-detector distance. The motion controller (Thorlabs, Inc.) controls the 2D movement of the detector fiber. (Zhu, 2008) 
Appendix F: Semi-solid phantom preparation

The phantom developed in this project consists of a breast shaped cup whose volume is occupied by a filler (Figure). The mold is made from polyurethane and can occupy a volume of $\sim 700 \mathrm{ml}$. The major axis of the cup is $16 \mathrm{~cm}$ and the minor axis is $11.5 \mathrm{~cm}$.

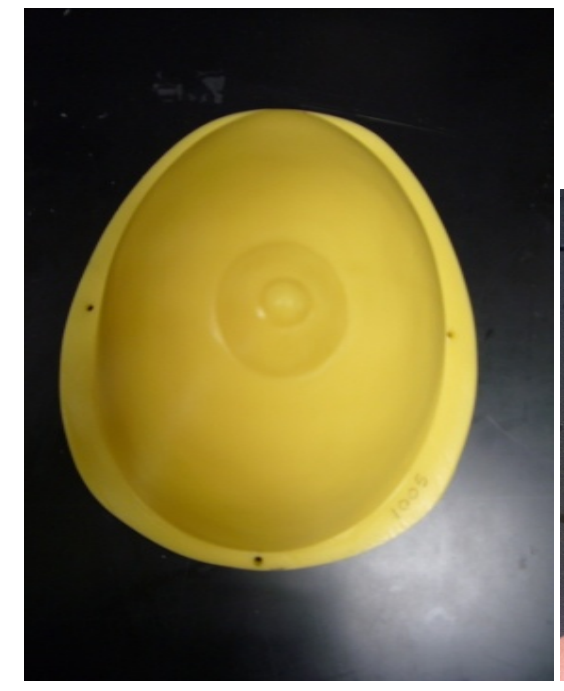

(a)

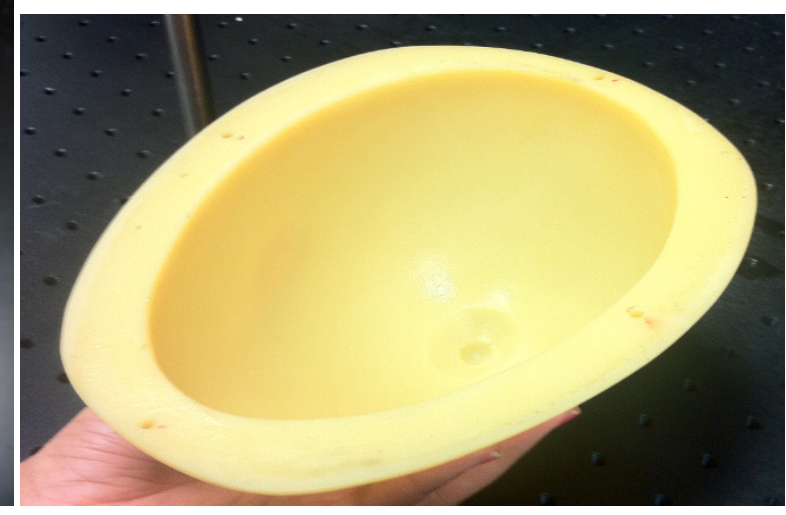

(b)

Figure 63: The breast shaped mold (provided by Imaging Diagnostic Systems Inc.) showing the (a) top view, and (b) internal hollow structure

The filler is composed of water and agar. Agar has a number of advantages: It is not affected by proteinases. It can retain its gelled characteristics even at room temperature. It holds the filler together and the phantom can retain its stability at room temperature. Liposyn is used to induce scattering characteristics. Preservatives like potassium metabisulfite and potassium sorbate are added to the filler to prevent bacterial growth. These preservatives increase the shelf life of the filler.

First, $700 \mathrm{ml}$ of distilled water is heated till bubbles are seen in it. Then $20 \mathrm{~g}$ of agar powder (NOW agar powder from Harvest Moon Health Foods) is added to it gradually, stirring as the mixture thickens. When it cools slightly above room temperature (roughly 5 minutes 
later), one teaspoon each of potassium metabisulfite (WineGuySupply.com) and potassium sorbate ( $\mathrm{R}$ and B's Wine Supply) is added. These prevent the yellowing of the phantom and thus, allow storage for a long time. Then $70 \mathrm{ml}$ of $20 \%$ liposyn is added and the mixture is stirred and poured into the breast shaped mold and allowed to set. The setting time is 120 minutes. After the phantom is set, it slides out easily from the mold when the mold is turned over to the other side [47, 53-55]. 


\section{References:}

1. D. Grosenick, H. Wabnitz, H. H. Rinneberg, K. T. Moesta, P. M. Schlag, "Development of a time domain optical mammograph and first in vivo applications," Appl. Opt., 38: 2927-2943 (1999).

2. S. B. Colak, M. B. van der Mark, G. W. 't Hooft, J. H. HooGenraad, E. S. van der Linden, F. A. Kuijpers, "Clinical Optical Tomography and NIR Spectroscopy for Breast Cancer Detection," IEEE Journal of Selected Topics in Quantum Electronics, 5: 1143-1158 (1999).

3. B. W. Pogue, S. P. Poplack, T. O. McBride, W. A. Wells, K. S. Osterman, U. L. Osterberg, K. D. Paulsen, "Quantitative hemoglobin tomography with diffuse near-infrared spectroscopy: Pilot results in the breast," Radiology, 218: 261-266 (2001).

4. H. Jiang, Y. Xu, N. Iftimia, J. Eggert, K. Klove, L. Baron, L. Fajardo, "Three-dimensional optical tomographic imaging of breast in a

human subject," IEEE Trans. Med. Imaging, 20: 1334-1340 (2001).

5. A. Li, G. Boverman, Y. Zhang, D. Brooks, E.L. Miller, M.E .Kilmer, Q. Zhang, E.M. Hillman, D.A. Boas, "Optimal linear inverse solution with multiple priors in diffuse optical tomography," Appl Opt. 44(10):1948-56 (2005).

6. A.P. Gibson, J.C. Hebden, S.R. Arridge, "Recent advances in diffuse optical imaging," Phys Med Biol. 50(4):R1-43(2005).

7. S. J. Erickson, A. Godavarty. Hand-held based near-infrared optical imaging devices: A review. Medical Engineering \& Physics 2009, 31, 495-509.

8. S. J. Erickson, A. Godavarty, S. Martinez, J. Gonzalez, A. Romero, M. Roman, A. Nunez, J. Ge, S. Regalado, R. Kiszonas, C. Lopez-Penalver. Hand-Held Optical Devices For Breast Cancer: Spectroscopy And 3D Tomographic Imaging. IEEE Journal of Selected Topics in Quantum Electronics 2011, 99, 1.

9. Q. Zhu, N.G. Chen, D. Q. Piao, P. Y. Guo, X. H. Ding, Design of near-infrared imaging probe with the assistance of ultrasound localization. Applied. Optics 2001, 40, 3288-3303.

10. B. J. Tromberg. Optical scanning and breast cancer. Academic Radiology 2005, 12, 923-924.

11. T. H. Pham, O. Coquoz, J. B. Fishkin, E. Anderson, B. J. Tromberg. Broad bandwidth frequency domain instrument for quantitative tissue optical spectroscopy. Review of Scientific Instruments 2000, 71, 2500-2513.

12. M. J. Holboke, B. J. Tromberg, X. Li, N. Shah, J. Fishkin, D. Kidney, J, Butler, B. Chance, A. G. Yodh. Three-dimensional diffuse optical mammography with ultrasound localization in a human subject. Journal of Biomedical Optics 2000, 5, 237-247. 
13. A. E. Cerussi, A. J. Berger, F. Bevilacqua, N. Shah, D. Jakubowski, J. Butler, R. F. Holcombe, B. J. Tromberg. Sources of absorption and scattering contrast for near-infrared optical mammography. Academic Radiology 2001, 8, 211-218.

14. A. E. Cerussi, D. Jakubowski, N. Shah, F. Bevilacqua, R. Lanning, A. J. Berger, D. Hsiang, J. Butler, R. F. Holcombe, B. J. Tromberg. Spectroscopy enhances the information content of optical mammography. Journal of Biomedical Optics 2002, 7, 60-71.

15. D. B. Jakubowski, A. E. Cerussi, F. Bevilacqua, N. Shah, D. Hsiang, J. Butler, B. J. Tromberg. Monitoring neoadjuvant chemotherapy in breast cancer using quantitative diffuse optical spectroscopy: a case study. Journal of Biomedical Optics 2004, 9, 230-238.

16. A. Cerussi, D. Hsiang, N. Shah, R. Mehta, A. Durkin, J. Butler, B. J. Tromberg. Predicting response to breast cancer neoadjuvant chemotherapy using diffuse optical spectroscopy. Proceedings of National Academy of Science USA 2007, 104, 4014-4019.

17. K. S. No, Q. Xie, P. H. Chou, R. Kwong, A. Cerussi, B. J. Tromberg. In vivo breast cancer measurement with a handheld laser breast scanner. in the 50th IEEE International Midwest Symposium on Circuits and Systems. August 5-8, Montreal Marriott Chateau Champlain Hotel (2007), MWSCAS.

18. B. Chance, S. Nioka, J. Zhang, E. F. Conan, E. Hwang, S. Briest, S. G. Orel, M. D. Schnall, B. J. Czerniecki. Breast cancer detection based on incremental biochemical and physiological properties of breast cancers: A six-year, two-site study. Academic Radiology 2005, 12, 925-933.

19. S. Nioka, B. Chance, NIR spectroscopic detection of breast cancer. Technology in Cancer Research and Treatment 2005, 4, 497-512.

20. B. Chance, Z. Zhao, S. Wen, Y. Chen. Simple ac circuit for breast cancer detection and object detection. Rev. Sci. Instr 2006, 77, (10), doi:10.1063/1.2205151:

http://rsi.aip.org/resource/1/rsinak/v77/i6/p064301_s1?isAuthorized=no

21. Q. Liu; Q. Luo; B. Chance, "2D phased array fluorescence wireless localizer in breast cancer detection." IEEE/EMBS International Summer School on Medical Devices and Biosensors (ISSS-MD), June 26 - July 2 2004, 71-73.

22. J. R. X. Xu, B. Qiang, J. J. Mao, S. P. Povoski. Development of a handheld near infrared imager for dynamic characterization of in vivo biological tissue systems. Applied Optics 2007 $46,7442-7451$.

23. T. Durduran, R. Choe, G. Yu, C. Zhou, J. C. Tchou, B. J. Czerniecki, Yodh AG. Diffuse optical measurement of blood flow in breast tumors. Optical Letters 2005, 30, 2915-2917.

24. A. Liebert, H. Wabnitz, J. Steinbrink, M. Moller, R. Macdonald, H. Rinneberg, A. Villringer, H. Obrig. Bed-side assessment of cerebral perfusion in stroke patients based on optical monitoring of a dye bolus by time-resolved diffuse reflectance. NeuroImage 2005, 24, 426-435. 
25. U. Sunar, H. Quon, T. Durduran, J. Zhang, J. Du, C. Zhou, G. Yu, R. Choe, A. Kilger, R. Lustig, L. Loevner, S. Nioka, B. Chance, A. G. Yodh. Noninvasive diffuse optical measurement of blood flow and blood oxyGenation for monitoring radiation therapy in patients with head and neck tumors: a pilot study. Journal of Biomedical Optics 2006, 11,(6): doi:10.1117/1.2397548. http://www.ncbi.nlm.nih.gov/pubmed/17212544

26. Q. Zhu, T. Durduran, V. Ntziachristos, M. Holboke, A. G. Yodh. Imager that combines nearinfrared diffusive light and ultrasound. Optical Letters 1999, 24, 1050-1052.

27. Q. Zhu, M. Huang, N. G. Chen, K. Zarfos, B. Jagjivan, M. Kane, P. Hedge, S. H. Kurtzman. Ultrasound-guided optical tomographic imaging of malignant and benign breast lesions: initial clinical results of 19 cases. Neoplasia 2003, 5, 379-388.

28. N. G. Chen, M. Huang, H. Xia, D. Piao. Portable near-infrared diffusive light imager for breast cancer detection. Journal of Biomedical Optics 2004, 9, 504-510.

29. B. Jayachandran, J. Ge, S. Regalado, A. Godavarty, "Design and development of a hand-held optical probe toward fluorescence diagnostic imaging," J. Biomed. Opt., 12(5): 054014 (2007).

30. J. Ge, B. Zhu, S. Regalado, A. Godavarty, "Three-dimensional fluorescence-enhanced optical tomography using a hand-held probe based imaging system," Med. Phys., 35(7): 3354-3363 (2008).

31. Boas, D.A.; Brooks, D.H.; Miller, E.L.; DiMarzio, C.A.; Kilmer, M.; Gaudette, R.J.; Quan Zhang; , "Imaging the body with diffuse optical tomography," Signal Processing Magazine, IEEE , vol.18, no.6, pp.57-75, Nov 2001

doi: $10.1109 / 79.962278$

32. L. V. Wang, H. Wu. Biomedical Optics: Principles and Imaging. Wiley, Hoboken , New Jersey, 2007.

33. G.Strangman, D. A. Boas, and J. P. Sutton, Non-Invasive Neuroimaging Using Near-Infrared Light BIOL PSYCHIATRY page 681 2002;52:679-693

34. J. Accardo, H. Kammann, A.H. Hoon, "Neuroimaging in cerebral palsy" The Journal of Pediatrics August 2004

35.A. Godavarty " Fluorescence Enhanced Optical Tomography on Breast Phantoms Using an Intensified CCD Imaging System." Texas A\&M, 2003

36. R. J. Grable, S. Ponder, S. Gardner, P. Jackewicz, J. Porter 'Phantom for Optical and Magnetic Resonance Imaging Quality Control' (United States Patent 6,675,035 B1, Jan 6, 2004)

37. J. C. Hebdeny, S. R. Arridgez and D. T. Delpyy." Optical imaging in medicine: I. Experimental techniques". Phys. Med. Biol. 42 (1997) 825-840. 
38. J Gonzalez, J DeCerce, S J Erickson, S L Martinez, A Nunez, M Roman, B Traub, C A Flores, S M Roberts, E Hernandez, W Aguirre, R Kiszonas2, A Godavarty." Hand-Held Optical Imager (Gen-2): Improved instrumentation and target detectability". Journal of Biomedical Optics (accepted - unpublished).

39. J. Gonzalez, M. Roman, M. Hall, and A.Godavarty. Gen-2 Hand-Held Optical Imager towards Cancer Imaging: Reflectance and Transillumination Phantom Studies. Sensors 2012, 12, 1-x manuscripts; doi:10.3390/s120x0000x

40. Weatherall, P., Evans, G. F., Metzger, G. J., Saborrian, M. H. and Leitch, A. M. (2001), MRI vs. histologic measurement of breast cancer following chemotherapy: Comparison with X-ray mammography and palpation. J. Magn. Reson. Imaging, 13: 868-875. doi: 10.1002/jmri.1124

41. Heron, D. E., Komarnicky, L. T., Hyslop, T., Schwartz, G. F. and Mansfield, C. M. (2000), Bilateral breast carcinoma. Cancer, 88: 2739-2750. doi: 10.1002/10970142(20000615)88:12<2739::AID-CNCR12>3.0.CO;2-J

42. $\mathrm{H} \mathrm{Xu}$ and $\mathrm{M}$ Patterson, "Determination of the optical properties of tissue-simulating phantoms from interstitial frequency domain measurements of relative fluence and phase difference," Opt. Express 14: 6485-6501 (2006).

43. F Martelli and G Zaccanti, "Calibration of scattering and absorption properties of a liquid diffusive medium at NIR wavelengths. CW method," Opt. Express 15: 486-500 (2007).

44. S J Madsen, M S Patterson and B C Wilson." The use of India ink as an optical absorber in tissue-simulating phantoms". Phys. Med. Biol. (1992) 37985 doi:10.1088/0031-9155/37/4/012

45. Victor Chernomordik, David W. Hattery, Amir Gandjbakhche, Dirk Grosenick, Heidrun Wabnitz, Herbert Rinneberg, K. Thomas Moesta and Peter M. Schlag. "Quantification of optical properties of a breast tumor using random walk theory" J. Biomed. Opt. 7, 80 (2002); http://dx.doi.org/10.1117/1.1427049

46. D R Leff, O J Warren, L C Enfield, A Gibson, T Athanasiou, D K. Patten, J Hebden, G Z Yang, A Darzi, "Diffuse optical imaging of the healthy and diseased breast: A systematic review," Breast Cancer Res Treat 108: 9-22 (2008). DOI 10.1007/s10549-007-9582-z

47. P.Joshi " 'Agar-based breast phantoms for imaging studies using a hand-held optical imager'" FIU 2011

48. Hsiang D, Shah N, Yu H, Su MY, Cerussi A, Butler J, et al. Coregistration of dynamic contrast enhanced MRI and broadband diffuse optical spectroscopy for characterizing breast cancer. Technol Cancer Res Treat 2005;4(5):549-58. 
49. Chance B, Nioka S, Zhang J, Conant EF, Hwang E, Briest S, et al. Breastcancer detection based on incremental biochemical and physiological properties of breast cancers: a six-year, twosite study. Acad Radiol 2005;12(8):925-33.

50. Chance B, Zhao Z, Wen S, Chen Y. Simple ac circuit for breast cancer detection and object detection. Rev Sci Instrum 2006;77:064301.

51. Nioka S, Chance B. NIR spectroscopic detection of breast cancer. Technol Cancer Res Treat 2005;4(5):497-512.

52. J. P. Culver, R. Choe, M. J. Holboke, L. Zubkov, T. Durduran, A. Slemp,, A. G. Yodh, V. Ntziachristos and B. Chance. "Three-dimensional diffuse optical tomography in the parallel plane transmission geometry: Evaluation of a hybrid frequency domain/continuous wave clinical system for breast imaging" Med. Phys. 30, 235 (2003); http://dx.doi.org/10.1118/1.1534109 (13 pages).

53. B. Zhu, S. Regalado, V. Sueiras, T. Huong, S. Nguyen, S.L. Ponder, A. Godavarty. 'Scattering Characterization of TiO2/Polyurethane Phantom Using Frequency-Domain Optical (Conference Paper: Biomedical Optics, 2008)

54. R. Srinivasan, D. Kumar and M. Singh. 'Optical Tissue- Equivalent Phantoms for Medical Imaging' (Trends in Biomaterials and Artificial Organs, Vol. 5, No.2, 2002)

55. V. G. Peters, D. R. Wyman, M. S. Patterson and G L Frank. "Optical properties of normal and diseased human breast". Physics in Medicine and Biology, 990, volume 35, Page 37 